\title{
SOLUTIONS OF THE NONLINEAR DIFFUSION EQUATION: \\ EXISTENCE, UNIQUENESS, AND ESTIMATION
}

by

John Howard Knight

A thesis submitted for the degree of Doctor of Philosophy

at the Australian National University

Canberra, April 1973 
Unless it is stated in the text to the contrary, the material contained in this thesis is the product of my own research.

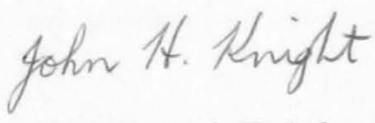

John Howard Knight 


\section{ACKNOWLEDGEMENTS}

I would like to thank the Australian National University for the award of a research scholarship, and Dr John Philip and Mrs Masako Izumi for their supervision. In particular I thank Dr Philip for many informative discussions, and constant encouragement and advice. I owe much to Dr Calvin Rose for developing my interest in this area, and to Dr Norman Dancer for discussions and helpful advice. 
ABSTRACT

In the first part of the thesis a problem for the nonlinear diffusion equation with diffusion coefficient a function of concentration, which is reduced by a similarity substitution to a boundary value problem for a nonlinear ordinary differential equation, is considered. Existence of a solution with certain upper and lower bounds is demonstrated for diffusion coefficient satisfying a local Lipschitz condition, and uniqueness is proved for non-increasing diffusion coefficient. An iterative method of Crank and Henry for solving this problem is investigated and is proved to converge for non-decreasing diffusion coefficient, thus extending the existence result in this case. A perturbation method is used to derive a general series solution to the problem for a class of diffusion coefficients of power-law and exponential form.

More general problems are considered in the last two chapters of the thesis. It is shown that a particular nonlinear diffusion equation with flux boundary conditions can be transformed to a linear equation, and new exact solutions are given to various problems of practical interest involving this nonlinear diffusion equation. An iterative method proposed by Parlange to solve various problems for the nonlinear diffusion equation and related equations is investigated, and it is shown that the method of Parlange fails to converge. The problems are formulated as integral equations, and a new iterative method is described which gives accurate solutions with a minimum of iteration. 
CHAPTER 1 INTRODUCTION

1.0 The equations to be solved

1.1 Exact solutions

1.2 Approximate solutions

CHAPTER 2 EXISTENCE, UNIQUENESS AND PROPERTIES OF SOLUTIONS FOR A TWO POINT BOUNDARY VALUE PROBLEM

2.0 Preliminary results

2.1 Uniqueness results

2.2 Existence and properties of solutions

CHAPTER 3 THE METHOD OF CRANK AND HENRY

3.0 The method

3.1 Preliminaries

3.2 Convergence proof for non-decreasing D

CHAPTER 4 AN ANALYTICAL SOLUTION BY PERTURBATION METHOD

4.0 Application of the Kirchhoff transformation

4.1 The perturbation expansion

CHAPTER 5 A NEW EXACT SOLUTION FOR A NONLINEAR DIFFUSION EQUATION WITH CONSTANT-FLUX BOUNDARY CONDITION

5.0 The method of Storm

5.1 Application of the method to more general problems

5.2 An exact solution corresponding to an instantaneous plane source

5.3 The solution to the general problem, and extensions to variable flux 
CHAPTER 6 CRITIQUE AND IMPROVEMENT OF PARLANGE'S ITERATIVE METHOD

6.0 The work of Parlange

6.1 The problem as an integral equation

6.2 Improvement of the Parlange method 


\subsection{THE EQUATIONS TO BE SOLVED}

The mathematical theory of diffusion may be described as the study of solutions of the parabolic partial differential equation

$$
\frac{\partial \theta}{\partial t}=\nabla \cdot(D \nabla \theta)
$$

where the dependent variable $\theta$ is the concentration of diffusing substance, and $D$ is the diffusion coefficient, which may be a function of the independent variables and of the dependent variable $\theta$. When $D$ varies with $\theta$, we have the nonlinear diffusion equation.

The diffusion equation (1) is a special case of the heat equation

$$
C \frac{\partial T}{\partial t}=\nabla \cdot(k \nabla T),
$$

where $\mathrm{T}$ is the temperature, and $\mathrm{C}$ and $\mathrm{k}$ are respectively the heat capacity and thermal conductivity of the material, which may be functions of the independent variables and of the temperature.

In particular the nonlinear diffusion equation may be applied to the movement of water in soils, when the influence of gravity may be neglected. When gravity must be taken into account, the process is described by the nonlinear Fokker-Planck equation

$$
\frac{\partial \theta}{\partial t}=\nabla \cdot(D \nabla \theta)+\frac{d K}{d \theta} \cdot \frac{\partial \theta}{\partial z},
$$

where $z$ is the vertical ordinate, and the coefficient $d K / d \theta$ is a function of $\theta$ (Klute, 1952a, 1952b; Philip, 1954, 1955a, 1957). The nonlinearity of the equations (2) and (3) is important in soil-water applications, as both coefficients $D$ and $d K / d \theta$ may depend very strongly on $\theta$. Typically, 
D may vary through two or three decades, and $\mathrm{dK} / \mathrm{d} \theta$ through several more (Philip, 1969).

In most problems considered here it will be required to find a solution of equation (1) in a semi-infinite one-dimensional region, with certain initial conditions, and subject to boundary conditions of either the concentration type, in which the concentration is specified on the boundary, or of the flux type, in which the flux -D $\partial \theta / \partial n$ is specified on the boundary, where $\mathrm{D}$ is the diffusion coefficient, and $\partial \theta / \partial \mathrm{n}$ denotes differentiation in a direction normal to the boundary. The thermal parameters $\mathrm{C}$ and $\mathrm{k}$ will be functions of temperature only, and the diffusion coefficient will be a function only of concentration $\theta$.

In this case where the coefficients are independent of time and position, equations (1) and (2) may be reduced to the same form by a transformation of Kirchhoff (1894). Putting

$$
\mathbf{s}(\theta)=\frac{1}{\mathrm{D}\left(\theta_{0}\right)} \int_{\theta_{0}}^{\theta} \mathrm{D}(\mathrm{a}) \mathrm{da},
$$

where $\theta_{0}$ is a datum value of $\theta$, transforms (1) to the form

$$
\frac{\partial s}{\partial t}=D(s) \nabla^{2} s
$$

where the diffusion coefficient has been expressed as a function of the new variable s. Similarly the transformation

$$
s(T)=\frac{1}{k\left(T_{0}\right)} \int_{T_{0}}^{T} k(a) d a
$$

reduces (2) to the similar form

$$
\frac{\partial s}{\partial t}=k(s) \nabla^{2} s
$$

Here $k$ is the thermal diffusivity, $k=k / C$, expressed as a function of $s$. Therefore without loss of generality we may use (5) as the general equation describing both heat conduction and diffusion. As noted by Carslaw and Jaeger (1959), when $\mathrm{k}(\mathrm{T})$ and $\mathrm{C}(\mathrm{T})$ are proportional, the 
thermal diffusivity is constant, and equation (6) is a linear equation. Even when the thermal diffusivity may be taken to be constant in this manner, if the thermal conductivity $k(T)$ is not constant we must apply the inverse of the transformation to the solution of the linear equation (6), in order to obtain the true temperature distribution. This was pointed out in a particular case by Awbery (1936). Where $\theta$ has upper and lower limits $\theta_{0}$ and $\theta_{1}$ respectively, we may conveniently put

$$
\theta(\theta)=\frac{\int_{\theta_{0}}^{\theta} D(a) d a}{\int_{\theta_{0}}^{\theta_{1}} D(a) d a}
$$

which allows the non-dimensional variable $\theta$ to range between 0 and 1 . The Kirchhoff transformation has the added advantage that it reduces a nonlinear flux-type boundary condition of the form

$$
D(\theta) \frac{\partial \theta}{\partial x}=-f \quad \text { when } x=a
$$

to a linear boundary condition

$$
\frac{\partial \Theta}{\partial x}=-g \text { when } x=a,
$$

where $\mathrm{f}$ and $\mathrm{g}$ are constants.

When $D(\theta)$ is positive for all $\theta$, then $\theta$ is a monotone function of $\theta$. This means that when $D(\theta)$ is a monotone function of $\theta$, and $D$ is positive as required in the physical context, $D(\theta)$ also has a monotonicity property.

The one-dimensional form of the diffusion equation is

$$
\frac{\partial}{\partial x}\left(D(\theta) \frac{\partial \theta}{\partial x}\right)=\frac{\partial \theta}{\partial t}
$$

and the Kirchhoff transformation reduces this to

$$
D(s) \frac{\partial^{2} s}{\partial x^{2}}=\frac{\partial s}{\partial t} \text {. }
$$

When the initial and boundary conditions are functions of $\mathrm{xt}^{-\frac{1}{2}}$, the substitution of Boltzmann (1894)

$$
\phi=x t^{-\frac{1}{2}}
$$


reduces equations (7) and (8) respectively to

$$
\frac{d}{d \phi}\left(D(\theta) \frac{d \theta}{d \phi}\right)+\frac{\phi}{2} \frac{d \theta}{d \phi}=0
$$

and

$$
\frac{d^{2} s}{d \phi^{2}}+\frac{\phi}{2 D(s)} \frac{d s}{d \phi}=0
$$

One set of initial and boundary conditions enabling the Boltzmann substitution to be used consists of the initial condition

$$
\theta(x, t)=\theta_{0} \text { when } t=0, x \geq 0
$$

and the constant-concentration boundary condition

$$
\theta(x, t)=\theta_{1} \text { when } t \geq 0, x=0 .
$$

These conditions become the boundary conditions

$$
\begin{aligned}
& \theta=\theta_{1} \text { when } \phi=0 \\
& \theta \rightarrow \theta_{0} \text { as } \phi \rightarrow \infty
\end{aligned}
$$

for the ordinary differential equation (10).

\subsection{EXACT SOLUTIONS}

Most of the monograph of Carslaw and Jaeger (1959) is concerned with the wide variety of methods of solution of the linear equation obtained from (1) or (2) when the diffusion coefficient or thermal parameters are constant. Very few exact solutions are known for equations with non-constant diffusivity.

Storm (1951) showed that the one-dimensional heat equation in a semi-infinite region with flux specified on the boundary may be reduced to a linear equation, when the thermal parameters satisfy a certain relation. His results were used by Friedmann (1956, 1957), but seem to have escaped the notice of later workers in the field, possibly because of the complicated form in which his criterion is expressed. In Chapter 5 it is shown that Storm's method provides a general exact solution to the 
one-dimensional diffusion equation with constant-flux boundary conditions and given initial concentration distribution, when the diffusion coefficient has the form $D(\theta)=a /(1-b \theta)^{2}$.

Fujita (1952a, 1952b, 1954) found exact solutions to the problem described by equation (10) and conditions (14), when the diffusion coefficient is of the form $a /(1-b \theta), a /(1-b \theta)^{2}$, or $a /\left(1+2 b \theta+c \theta^{2}\right)$. Fujita's solution to this problem with $D(\theta)=a /(1-b \theta)^{2}$ may in fact be deduced by the method of Storm applied to the flux-type boundary condition problem with flux specified on the boundary to be proportional to $t^{-\frac{1}{2}}$.

Philip (1960a, 1960b) found a very general method for finding $D(\theta)$ functions which yield exact solutions to equation (10) with condition (14)

\subsection{APPROXIMATE SOLUTIONS}

The lack of exact solutions to the above problem, when $D(\theta)$ has simple exponential or polynomial forms, led to a search for approximate methods of solution. These may be divided into several classes.

Various analytical solutions can be obtained by perturbation methods, as power series in a "small" parameter. They are approximate because computational difficulties usually mean that only the first few terms of the series can be easily calculated, although in theory all terms can be calculated explicitly with sufficient labour, and each such series has a certain region of convergence. Kirchhoff and Hansemann (1880) were apparently the first to apply such a method to this problem. Hopkins (1938) calculates the first few terms of the solution of the corresponding heat flow problem, for the case where the conductivity and the heat capacity are both quadratic functions of the temperature. Clarke (1957) 
calculated a series solution to the diffusion problem, equation (10) with conditions (14), when $D(\theta)=D_{0} \exp (a \theta)$. Kidder (1957) applied the Kirchhoff transformation, and then calculated a series solution to the problem with $D(\theta)=a(1+b \theta)$. In Chapter 4 a general perturbation expansion is obtained which covers the cases $D(\theta)=a(1+b \theta)^{k}$ with $k>0$, and $D(\theta)=D_{0} \exp (c \theta)$. Kidder's series can be written down as a particular case of our more general expansion. Yampol'skii and Aizen (1968) considered the heat conduction problem with thermal conductivity and heat capacity $\mathrm{k}_{0}(1+\mathrm{aT})$ and $\mathrm{C}_{0}(1-\mathrm{aT})$ respectively. All the above analytical methods involve perturbation about the case of constant $D$, and so may be expected to be most accurate when $D(\theta)$ does not vary too widely with $\theta$, and to be less useful for strongly nonlinear equations.

Solutions may also be obtained by quasi-analytical iterative methods, such as the method of successive approximations, in which an estimate of the solution is used to give another, hopefully better, estimate of the solution. The process often uses a Green's function and an integral operator to give an expression in closed form for the new estimate in terms of the old estimate. In practice the quadratures involved must often be performed using a suitable scheme for numerical integration, when a numerical answer is required.

Crank and Henry (1949a, 1949b) developed a method of successive approximations for solving (10) or (11) with boundary conditions (14). In Chapter 2, various properties such as existence, uniqueness and bounds for solutions are investigated for this problem, using mainly the techniques of Bailey, Shampine, and Waltman (1968). This work is extended in Chapter 3 to show that the iteration scheme of Crank and Henry converges when $D(\theta)$ satisfies certain monotonicity and continuity conditions, and gives a unique solution when $D(\theta)$ has in addition a smoothness property. As with all such schemes, the speed of convergence 
of the method of Crank and Henry may be increased by careful choice of the starting function.

Klute $(1952 \mathrm{a}, 1952 \mathrm{~b})$ used the method of Crank and Henry under conditions of very rapid variation of $\mathrm{D}$ with $\theta$, and found that convergence was slow under these conditions. Philip (1955b) developed a much more rapid and accurate numerical method which used an inverted form of equation (10), with an analytical approximation near the point $\theta=\theta_{0}$. Philip (1957) made this method the basis of a series solution to an inverted form of equation (3). Recently, Parlange (1971a, 1971b, 1971c) has proposed a new iterative method of solving equations (1) and (3) subject to concentration-type boundary conditions. Parlange's method is investigated in Chapter 6 , where it is shown that for the problem described by equation (7) subject to conditions (12) and (13), it is equivalent to the application of the method of successive approximations of Picard (1893) to a two-point boundary value problem for a particular nonlinear ordinary differential equation. In fact the iterates do not converge in the conventional sense, but merely oscillate about the true solution (Knight and Philip, 1973). Philip and Knight (1973) developed an improved iteration scheme for which each iterate satisfies a constraint of "integral continuity", and the characteristic oscillation of Parlange's method is eliminated. A more general choice of first approximation is made to suit the particular problem. An improved iteration scheme is developed for the infiltration equation (3) with conditions (12) and (13), which avoids the serious defects of Parlange's method. A good first approximation makes iteration unnecessary.

Also in the class of iterative solutions is the work of Mann and Wolf (1951) who showed that for a linear heat equation in a onedimensional semi-infinite region, with certain nonlinear mixed boundary conditions of "radiation" type, Laplace transform methods could be used 
to derive a nonlinear integral equation involving the surface temperature. They gave conditions under which this integral equation could be solved by the method of successive approximations, thus eliminating the need to calculate the temperature distribution in the whole region in order to know the surface temperature and flux. Abarbanel (1960) extended their work to the sphere and to the finite region.

A further class of approximate solutions includes the rest of those which do not exactly satisfy the equation and boundary conditions, but are "near" the true solution in some sense. They may be improved by correct choice of parameters, but there is no general systematic procedure for using a first approximation to construct a "better" approximation to the solution. Some examples can be given.

Landah1 (1953) gave an approximation method for transient diffusion with constant D, and applied it to various boundary value problems. Macey (1959) generalized this approach to non-constant diffusion coefficients, using a "moving steady-state" solution, and found quite good agreement with existing exact solutions. Goodman (1958)

approximated temperature profiles by polynomials, whose coefficients were chosen to satisfy certain constraints, chief of which being one of overall conservation, referred to by Goodman as the "heat-balance integral". In Goodman (1961) he extended the method to variable diffusion coefficients, and compared the approximations to solutions obtained by the method of Crank and Henry, as rediscovered by Yang (1958).

The conclusion we are forced to draw from a study of all the above approximate methods of solution of the diffusion equation is that a general quasi-analytic approximate method of solution still awaits discovery. With many of the above authors, the first or only problem considered for solution is one which is reduced by the Boltzmann substitution to a simple two-point boundary value problem for an ordinary 
differential equation. Consequently the limitations of all the above methods when applied to more general problems are not clearly brought out. Although analytical methods must be used wherever possible to explore the fundamental structure of solutions, it seems that for many problems of interest the only hope of obtaining reasonably accurate numerical answers lies in the use of purely numerical finite-difference methods on highspeed computers. 
CHAPTER 2

EXISTENCE, UNIQUENESS, AND PROPERTIES OF SOLUTIONS

FOR A TWO-POINT BOUNDARY VALUE PROBLEM

\subsection{PRELIMINARY RESULTS}

In this chapter we consider the one-dimensional diffusion equation

$$
\frac{\partial \theta}{\partial t}=\frac{\partial}{\partial x}\left(D(\theta) \frac{\partial \theta}{\partial x}\right)
$$

in the semi-infinite region defined by the condition $x \geq 0$, with initial and boundary conditions

$$
\theta=\theta_{0} \text { when } t=0, x>0 ; \quad \theta=\theta_{1} \text { when } x=0, t \geq 0 \text {. }
$$

The function $D(\theta)$ is defined and positive when $\theta$ lies between $\theta_{0}$ and $\theta_{1}$. For physical reasons we require that the solution $\theta(x, t)$ of $(15)$ be bounded by $\theta_{0}$ and $\theta_{1}$.

The following uniqueness theorem is a particular case of a result of Seyferth (1962).

Theorem 1 (Seyferth) When $\mathrm{D}(\theta)$ is continuous and positive and has a continuous derivative $\mathrm{dD} / \mathrm{d} \theta$ in $\left[\theta_{0}, \theta_{1}\right]$, the above problem has at most one bounded solution.

We apply the Kirchhoff transformation in the form

$$
s(\theta)=\frac{\int_{\theta_{0}}^{\theta} D(a) d a}{\int_{\theta_{0}}^{\theta_{1}} D(a) d a} .
$$

The equation satisfied by $s$ is

$$
\frac{\partial s}{\partial t}=D(s) \frac{\partial^{2} s}{\partial x^{2}}
$$

subject to conditions 


$$
s=0 \text { when } t=0, x>0 ; \quad s=1 \text { when } x=0, t \geq 0 \text {. }
$$

The Boltzmann substitution $\phi=x t^{-\frac{1}{2}}$ reduces this to the ordinary differential equation

$$
\frac{d^{2} s}{d \phi^{2}}+\frac{\phi}{2 D(s)} \frac{d s}{d \phi}=0
$$

subject to the boundary conditions

$$
s=1 \text { when } \phi=0 ; \quad s \rightarrow 0^{\prime} \text { as } \phi \rightarrow \infty \text {. }
$$

Now that we have reduced our problem to a two-point boundary value problem for an ordinary differential equation, we may consider questions of existence and uniqueness, and examine the properties of solutions, using the simpler techniques of ordinary differential equations, such as those of Bailey, Shampine, and Waltman (1968).

We need some well-known results about solutions of initial-value problems for ordinary differential equations, found for example in Birkhoff and Rota (1969), or in Bailey, Shampine, and Waltman (1968).

Definition A function $\mathrm{f}\left(\phi, \mathrm{y}, \mathrm{y}^{\prime}\right)$ satisfies a Lipschitz condition in a domain $\mathrm{G}$ if and only if there exist two non-negative constants $\mathrm{K}$ and $\mathrm{L}$ such that whenever $\left(\phi, y_{1}, y_{1}^{\prime}\right)$ and $\left(\phi, y_{2}, y_{2}^{\prime}\right)$ are in $G$, the inequality

$$
\left|f\left(\phi, y_{1}, y_{1}^{\prime}\right)-f\left(\phi, y_{2}, y_{2}^{\prime}\right)\right| \leq K\left|y_{1}-y_{2}\right|+L\left|y_{1}^{\prime}-y_{2}^{\prime}\right|
$$

holds.

Theorem 2 (Bailey et al., p.10) Local Existence of Solutions of Initial value Problems.

If $\mathrm{f}\left(\phi, \mathrm{y}, \mathrm{y}^{\prime}\right)$ is continuous in a domain $\mathrm{G}$ which contains the point $\left(\phi_{0}, \mathrm{y}_{0}, \mathrm{y}_{0}^{\prime}\right)$, then there exists at least one twice continuously differentiable function $y(t)$, defined on some interval I containing $\phi_{0}$, which satisfies

$$
y^{\prime \prime}(\phi)+f\left(\phi, y(\phi), y^{\prime}(\phi)\right)=0
$$




$$
y\left(\phi_{0}\right)=y_{0}, \quad y^{\prime}\left(\phi_{0}\right)=y_{0}^{\prime},
$$

and has $\left(\phi, y(\phi), y^{\prime}(\phi)\right)$ in $G$ for all $\phi$ in $I$.

Theorem 3 (Bailey et al., p.10) Uniqueness and Continuability of Solutions of Initial Value Problems.

If $\mathrm{f}\left(\phi, y, \mathrm{y}^{\prime}\right)$ is continuous and satisfies a Lipschitz condition in a domain $G$ which contains the point $\left(\phi_{0}, \mathrm{y}_{0}, \mathrm{y}_{0}^{\prime}\right)$, then there is only solution of (22) and (23), and this solution can be uniquely continued arbitrarily close to the boundary of $G$.

Theorem 4 (Bailey et al., p.11) Continuous Dependence of Solution on Initial Conditions and Parameter.

Let $G$ be a domain containing the point $\left(\phi_{0}, \mathrm{y}_{0}, \mathrm{y}_{0}^{\prime}\right)$, let $\mathrm{E}$ be an open interval containing $\varepsilon_{0}$, and suppose that $f\left(\phi, y, y^{\prime}, \varepsilon\right)$ is a continuous function on $\mathrm{D} \times \mathrm{E}$, bounded by a constant there. Let $\mathrm{I}$ be a compact interval containing $\phi_{0}$ on which there exists a unique solution $y_{0}(\phi)$ of (22), (23) when $\varepsilon=\varepsilon_{0}$. Then there exists a $\delta>0$ such that for any $(\mathrm{a}, \mathrm{A}, \mathrm{m}, \varepsilon) \in \mathrm{D} \times \mathrm{E}$ satisfying $\left|\mathrm{a}-\phi_{0}\right|+\left|\mathrm{A}-\mathrm{y}_{0}\right|+\left|\mathrm{m}-\mathrm{y}_{0}^{\prime}\right|+\left|\varepsilon-\varepsilon_{0}\right|<\delta$, all solutions of (22), (23) exist on I. If $y(t, a, A, m, \varepsilon)$ is any solution of $(22),(23)$ on $I$, then as $(a, A, m, \varepsilon) \rightarrow\left(\phi_{0}, y_{0}, y_{0}^{\prime}, \varepsilon_{0}\right)$,

$$
y(\phi, a, A, m, \varepsilon) \rightarrow y\left(\phi, \phi_{0}, y_{0}, y_{0}^{\prime}, \varepsilon_{0}\right)=y_{0}(\phi)
$$

and

$$
\mathrm{y}^{\prime}(\phi, \mathrm{a}, \mathrm{A}, \mathrm{m}, \varepsilon) \rightarrow \mathrm{y}^{\prime}\left(\phi, \phi_{0}, \mathrm{y}_{0}, \mathrm{y}_{0}^{\prime}, \varepsilon_{0}\right)=\mathrm{y}_{0}^{\prime}(\phi)
$$

uniformly on the interval $I$.

We also need the following comparison theorems from Bailey, Shampine and Waltman (1968).

Theorem 5 (Bailey et al., p.58) Position of Maxima of Solutions. Suppose $g(\phi)$ is a piecewise continuous function on $[a, b]$ and $u(\phi)$ are non-negative twice continuously differentiable functions satisfying 


$$
\begin{aligned}
& u^{\prime \prime}+g u^{\prime} \geq 0 \\
& v^{\prime \prime}+g v^{\prime} \leq 0
\end{aligned}
$$

on $(\mathrm{a}, \mathrm{b})$ with $\mathrm{u}\left(\phi_{0}\right)=\mathrm{v}\left(\phi_{0}\right)$ and $\mathrm{u}^{\prime}\left(\phi_{0}\right)=\mathrm{v}^{\prime}\left(\phi_{0}\right)>0$ for some $\phi_{0}$ in $[\mathrm{a}, \mathrm{b}]$.

Then $v^{\prime}(\phi)$ has a zero between $\phi_{0}$ and any zero of $u^{\prime}(\phi)$ to the right of $\phi_{0}$.

Theorem 6 (Bailey et al., p.81) Comparison Theorem.

Let $\mathrm{h}\left(\phi, \mathrm{u}, \mathrm{u}^{\prime}\right)$ be continuous on $[\mathrm{a}, \mathrm{b}] \times(-\infty, \infty) \times(-\infty, \infty)$ and such that the differential equation

$$
\mathrm{u}^{\prime \prime}(\phi)+\mathrm{h}\left(\phi, \mathrm{u}(\phi), \mathrm{u}^{\prime}(\phi)\right)=0
$$

has the properties that all initial value problems have unique solutions on $[a, b]$, and that two different solutions of (24) cannot agree in value at more than one point of $[a, b]$.

Let $\mathrm{v}(\phi)$ be a twice continuously differentiable function on [a,b] satisfying

$$
\mathrm{v}^{\prime \prime}(\phi)+\mathrm{h}\left(\phi, \mathrm{v}(\phi), \mathrm{v}^{\prime}(\phi)\right) \geq 0
$$

If $\mathrm{u}(\phi)$ is a solution of (24) which agrees with $\mathrm{v}(\phi)$ in both value and slope at some point $\phi_{0}$ in $[a, b]$, then

$$
v(\phi) \geq u(\phi) \text { for } \phi \text { in }[a, b] \text {. }
$$

The inequality signs may be reversed throughout.

We are now in a position to prove a theorem about the properties of the derivative of the solution of our equation (20) subject to conditions (21).

Theorem 7 Let $D(\theta)$ be defined and continuous for all $\theta$ in $\left[\theta_{0}, \theta_{1}\right]$, and such that there exists $D_{0}>0$ such that for all $\theta$ in $\left[\theta_{0}, \theta_{1}\right]$, $\mathrm{D}_{0} \leq \mathrm{D}(\theta)$

Let $s(\phi)$ satisfy the differential equation 


$$
\frac{\mathrm{d}^{2} \mathrm{~s}}{\mathrm{~d} \phi^{2}}+\frac{\phi}{2 \mathrm{D}(\mathrm{s})} \frac{\mathrm{ds}}{\mathrm{d} \phi}=0
$$

for $\phi$ in $[0, \infty)$, with boundary conditions

$$
\mathrm{s}(0)=1, \quad s(\infty)=0 \quad(21),
$$

with $s(\phi)$ in $[0,1]$ for all $\phi \geq 0$.

Then $\frac{\mathrm{ds}}{\mathrm{d} \phi}$ is non-zero for all $\phi \geq 0$.

$\underline{\text { Proof }}$

The hypotheses on $\mathrm{D}(\theta)$ mean that after the application of the Kirchhoff transformation, $1 / D(s)$ is continuous as a function of $s$, for $s$ in $[0,1]$.

Consider the function $\mathrm{u}(\phi)$ defined by

$$
\mathrm{u}(\phi)=1-\mathrm{s}(\phi) .
$$

The function $u(\phi)$ satisfies $u(0)=0$, and

$$
u^{\prime \prime}+\frac{\phi}{2 D(1-u(\phi))} u^{\prime}=0 \text {. }
$$

The unique ${ }^{1}$ solution of (25) with $u(0)=0, u^{\prime}(0)=0$ satisfies $u(\phi)=0$ for all $\phi \geq 0$. Therefore the solution of (25) with $u(0)=0, u(\infty)=1$ satisfies $u^{\prime}(0)=m$, for some $m>0$.

Suppose that the conclusion of the theorem is not true. Then there is a smallest $\phi_{1}$ such that $u^{\prime}\left(\phi_{1}\right)=0$, and $u^{\prime}(\phi) \geq 0$ when $0 \leq \phi \leq \phi_{1}$.

Since $\mathrm{D}(1-\mathrm{u}(\phi)) \geq \mathrm{D}_{0}>0$ and $\mathrm{u}^{\prime}(\phi) \geq 0$,

$$
\frac{\phi}{2 \mathrm{D}(1-\mathrm{u}(\phi))} \mathrm{u}^{\prime} \leq \frac{\phi}{2 \mathrm{D}_{0}} \mathrm{u}^{\prime} \text { when } 0 \leq \phi \leq \phi_{1} .
$$

Therefore

$$
0=u^{\prime \prime}+\frac{\phi}{2 D(1-u(\phi))} u^{\prime} \leq u^{\prime \prime}+\frac{\phi}{2 D_{0}} u^{\prime}
$$

Let $v(\phi)$ be the function satisfying

$$
v^{\prime \prime}+\frac{\phi}{2 D_{0}} v^{\prime}=0
$$

1 Unigeness can be established using an "integrating factor" argument. 


$$
\mathrm{v}(0)=0, \quad \mathrm{v}^{\prime}(0)=\mathrm{m} \text {. }
$$

Explicitly,

$$
v^{\prime}(\phi)=m \exp \left(-\phi^{2} / 4 D_{0}\right),
$$

which is never zero for any finite $\phi$.

By Theorem 5 on position of maxima of solutions, since $u^{\prime}\left(\phi_{1}\right)=0$ by hypothesis, and on $\left[0, \phi_{1}\right]$

$$
\begin{aligned}
& u^{\prime \prime}+\frac{\phi}{2 D_{0}} u^{\prime} \geq 0, \\
& v^{\prime \prime}+\frac{\phi}{2 D_{0}} v^{\prime}=0
\end{aligned}
$$

then $v^{\prime}(\phi)$ must have a zero on $\left[0, \phi_{1}\right]$, which is a contradiction.

Therefore $u^{\prime}(\phi)$ does not have a zero on $[0, \infty)$ and so $d s / d \phi$ is never zero for any finite $\phi$, and the theorem is proved.

The hypotheses on $D(\theta)$ in Theorem 7 ensure as a corollary that $d \theta / d \phi$ is non-zero, and that $\theta(\phi)$ is a strictly monotonic function of $\phi$.

\subsection{UNIQUENESS RESULTS}

For Chapter 3 we need to know how to solve certain boundary-value problems for linear equations. Uniqueness of these solutions follows from the theorem on positions of maxima of solutions.

Theorem 8 Uniqueness for Linear Boundary Value Problems.

Let $\mathrm{g}(\phi)$ be a piecewise continuous function of $\phi$ such that $D_{1} \geq \mathrm{g}(\phi) \geq \mathrm{D}_{0}>0$, for all $\phi \geq 0$. Then the problem

$$
\begin{gathered}
y^{\prime \prime}+\frac{\phi}{2 g(\phi)} y^{\prime}=0, \\
y(0)=1, \quad y(\infty)=0
\end{gathered}
$$

has one and only one solution. 


\section{Proof}

It may easily be verified that the function $y(\phi)$ defined by

$$
y(\phi)=\frac{\int_{\phi}^{\infty} \exp \left(-\int_{0}^{\mu} \frac{a}{2 g(a)} d a\right) d \mu}{\int_{0}^{\infty} \exp \left(-\int_{0}^{\mu} \frac{a}{2 g(a)} d a\right) d \mu}
$$

is a solution to (26) and (27).

Suppose that $\mathrm{u}(\phi)$ and $\mathrm{v}(\phi)$ are two different solutions. Then their difference $w(\phi)=u(\phi)-v(\phi)$ satisfies

$$
\mathrm{w}^{\prime \prime}+\frac{\phi}{2 \mathrm{~g}(\phi)} \mathrm{w}^{\prime}=0 ; \quad \mathrm{w}(0)=0, \quad \mathrm{w}(\infty)=0 .
$$

If $\mathrm{w}^{\prime}(0)=0$, then $\mathrm{w}(\phi)=0$ for all $\phi$, and $\mathrm{u}$ and $\mathrm{v}$ are identical.

Suppose that $\mathrm{w}^{\prime}(\phi)$ is non-zero. Without loss of generality, we may take $\mathrm{w}^{\prime}(0)=\mathrm{m}>0$.

An argument similar to that used in the proof of Theorem 7 shows that $w^{\prime}(\phi)$ is non-zero for all finite $\phi$, and so $w$ is a strictly increasing function, and cannot satisfy both of the conditions $w(0)=0$, $\mathrm{w}(\infty)=0$.

This contradiction shows that we must have $\mathrm{w}^{\prime}(0)=0$, hence $\mathrm{w}(\phi)=0$ for all $\phi$, and $\mathrm{u}$ and $\mathrm{v}$ are identical. this establishes the uniqueness of the above solution.

We do not expect to have uniqueness for the more general nonlinear equation (20) without some conditions on the function $D(\theta)$. The following uniqueness theorem requires $D(s)$ to be a non-increasing function of $s$ on $[0,1]$. For positive $D$ this condition holds when $\theta_{0}<\theta$, and $D(\theta)$ is a non-increasing function of $\theta$ on $\left[\theta_{0}, \theta_{1}\right]$, or when $\theta_{1}<\theta_{0}$ and $D(\theta)$ is a non-decreasing function of $\theta$ on $\left[\theta_{1}, \theta_{0}\right]$. The latter situation corresponds to a "desorption" problem. 
Theorem 9 Uniqueness Theorem for monotonic D.

Let $D(s)$ be a continuous function of $s$ defined on $[0,1]$, such that $D\left(s_{1}\right) \geq D\left(s_{2}\right)$ whenever $s_{1} \leq s_{2}$, and such that there exists $D_{0}>0$ with $\mathrm{D}(\mathrm{s}) \geq \mathrm{D}_{0}$ on $[0,1]$.

Then the equation

$$
s^{\prime \prime}+\frac{\phi}{2 D(s)} s^{\prime}=0
$$

with conditions

$$
\mathrm{s}(0)=1, \mathrm{~s}(\infty)=0
$$

has at most one solution such that $s(\phi) \in[0,1]$ for al1 $\phi \geq 0$.

\section{$\underline{\text { Proof }}$}

Let $\mathrm{y}_{1}, \mathrm{y}_{2}$ be two solutions of (20) satisfying (21). Then $\mathrm{u}(\phi)=\mathrm{y}_{1}(\phi)-\mathrm{y}_{2}(\phi)$ satisfies

and

$$
u^{\prime \prime}+\frac{\phi}{2}\left[\frac{u^{\prime}}{D\left(u+y_{2}\right)}+y_{2}^{\prime}\left(\frac{1}{D\left(u+y_{2}\right)}-\frac{1}{D\left(y_{2}\right)}\right)\right]=0
$$

$$
\mathrm{u}(0)=0, \quad \mathrm{u}(\infty)=0 .
$$

This has the trivial solution which is identically zero.

Suppose that there is another solution $\mathrm{u}$ which is not identically zero. Without loss of generality we may take $u^{\prime}(0)=m>0$. In order that we may have $u(\infty)=0$, it is necessary that $u^{\prime}(\phi)=0$ for some $\phi \in[0, \infty)$. Let $\phi_{1}$ be the smallest such $\phi$.

Then for $\phi \in\left[0, \phi_{1}\right], u(\phi) \geq 0$, and $u^{\prime}(\phi) \geq 0$. This means that

and

$$
y_{2}(\phi) \leq y_{1}(\phi),
$$

$$
y_{2}^{\prime}(\phi) \leq y_{1}^{\prime}(\phi)<0 \text {. }
$$

The monotonicity property means that

Therefore

$$
0<\mathrm{D}_{0} \leq \mathrm{D}\left(\mathrm{y}_{1}(\phi)\right) \leq \mathrm{D}\left(\mathrm{y}_{2}(\phi)\right) \text {. }
$$

$$
\frac{1}{D\left(y_{1}\right)} \geq \frac{1}{D\left(y_{2}\right)}
$$


so

$$
\frac{-\phi y_{2}^{\prime}}{2 D\left(y_{1}\right)} \geq \frac{-\phi y_{2}^{\prime}}{2 D\left(y_{2}^{-}\right)} \text {. }
$$

Therefore

$$
\left(y_{1}-y_{2}\right)^{\prime \prime}+\frac{\phi}{2 D\left(y_{1}\right)}\left(y_{1}-y_{2}\right)^{\prime} \geq y_{1}^{\prime \prime}+\frac{\phi y_{1}^{\prime}}{2 D\left(y_{1}\right)}-y_{2}^{\prime \prime}-\frac{\phi y_{2}^{\prime}}{2 D\left(y_{2}\right)}
$$

or

$$
u^{\prime \prime}+\frac{\phi}{2 D\left(y_{1}\right)} u^{\prime} \geq 0
$$

Since $D\left(y_{1}\right) \geq D_{0}$, and $u_{1} \geq 0$,

$$
u^{\prime \prime}+\frac{\phi}{2 D_{0}} u^{\prime} \geq 0
$$

As usual let $v(\phi)$ be the function satisfying $v(0)=0, v^{\prime}(0)=m$,

$$
\mathrm{v}^{\prime \prime}+\frac{\phi}{2 \mathrm{D}_{0}} \mathrm{v}^{\prime}=0
$$

Then $v^{\prime}(\phi)$ is non-zero for all finite $\phi$. This is a contradiction to the theorem on position of maxima of solutions, and so there is no finite $\phi_{1}$ such that $\mathrm{u}^{\prime}\left(\phi_{1}\right)=0$, and $\mathrm{u}$ is in fact identically zero, which establishes the uniqueness property.

It is interesting to note that no conditions such as Lipschitz or smoothness conditions are necessary to establish uniqueness when $D(s)$ is a monotonically decreasing function of s. For more general D, we impose additional conditions. The following theorem follows from the more general Theorem 1 .

Theorem 10 (Seyferth)

Let $\mathrm{D}$ be a continuous function of $\theta$ on $\left[\theta_{0}, \theta_{1}\right]$, with a continuous derivative $d D / d \theta$ there, and such that there is a $D_{0}>0$ with $D(\theta) \geq D_{0}$ for a11 $\theta$ in $\left[\theta_{0}, \theta_{1}\right]$. Then the equation

$$
s^{\prime \prime}+\frac{\phi}{2 D(s)} s^{\prime}=0
$$

with conditions $s(0)=1, s \rightarrow 0$ as $\phi \rightarrow \infty$, has at most one solution satisfying $s(\phi) \in[0,1]$ for all $\phi \geq 0$. 


\subsection{EXISTENCE AND PROPERTIES OF SOLUTIONS}

In order to prove the existence of a solution to equation (20) which satisfies conditions (21) by "shooting" from the end $\phi=0$, we need to use Theorems 2, 3 and 4. For these we need a Lipschitz condition to ensure uniqueness of solutions of initial value problems.

Let $D(s)$ be such that $D$ is continuous on $[0,1]$, and $D(s) \geq D_{0}>0$.

The function

$$
f\left(\phi, y, y^{\prime}\right)=\frac{\phi}{2 D(y)} y^{\prime}
$$

is defined and continuous on $[0, \infty) \times[0,1] \times[-\infty, \infty]$, but does not satisfy a Lipschitz condition there. To obtain a Lipschitz condition we must impose conditions on $D(\theta)$, and restrict the domain of $f$. The requirement that $D(\theta)$ be continuous and non-zero on $\left[\theta_{0}, \theta_{1}\right]$, which implies the existence of $D_{0}, D_{2}>0$ such that $0<D_{0} \leq D(\theta) \leq D_{1}$ for all $\theta$ in $\left[\theta_{0}, \theta_{1}\right]$, ensures that if $D(\theta)$ satisfies a Lipschitz condition as a function of $\theta$, then $\mathrm{D}(\mathrm{s})$ satisfies a Lipschitz condition as a function of $\mathrm{s}$. The above conditions on $D$ mean in particular that if $D(\theta)$ satisfies a Lipschitz condition on a closed interval $\left[\theta_{2}, \theta_{1}\right]$ not including $\theta_{0}$, then $D(s)$ satisfies a Lipschitz condition on a subinterval $[a, 1]$ of $[0,1]$, where $\mathrm{a}>0$.

If $\mathrm{D}(\mathrm{s})$ satisfies a Lipschitz condition on a subinterval $[a, 1]$ of $[0,1]$, and $\phi$ is restricted to a finite interval $[0, b]$, and $s$ to a finite interval $[-n, 0]$, then

$$
f\left(\phi, s, s^{\prime}\right)=\frac{\phi s^{\prime}}{2 D(s)}
$$

satisfies a Lipschitz condition on $[0, b] \times[a, 1] \times[-n, 0]$, and we know that the solution of the initial value problem

$$
\begin{gathered}
y(0)=1, \quad y^{\prime}(0)=m, \quad-n<m<0 \\
y^{\prime \prime}+\frac{\phi}{2 D(y)} y^{\prime}=0
\end{gathered}
$$


exist and are unique on the restricted domain, and depend continuously on the parameter $\mathrm{m}$ there, by Theorems 2,3 , and 4 . We are now in a position to prove the existence theorem for our boundary value problem. Without loss of generality we can assume that $\theta_{0}<\theta_{1}$.

\section{Theorem 11 Existence Theorem.}

Let $D(\theta)$ be defined and continuous on $\left[\theta_{0}, \theta_{1}\right]$, and non-zero there. Let $D(\theta)$ satisfy a Lipschitz condition in $\theta$ on any closed subinterval of $\left[\theta_{0}, \theta_{1}\right]$ not including $\theta_{0}$. Put $\underset{\theta}{\min }[\mathrm{D}(\theta)]=\mathrm{D}_{\min }>0, \underset{\theta}{\max }[\mathrm{D}(\theta)]=\mathrm{D}_{\max }<\infty$. Put

$$
s(\theta)=\frac{\int_{\theta_{0}}^{\theta} \mathrm{D}(\mathrm{a}) \mathrm{da}}{\int_{\theta_{0}}^{\theta_{1}} \mathrm{D}(\mathrm{a}) \mathrm{da}} .
$$

Then the boundary value problem

$$
\begin{gathered}
\frac{d}{d \phi}\left(D(\theta) \frac{d \theta}{d \phi}\right)+\frac{\phi}{2} \frac{d \theta}{d \phi}=0 \\
\theta(0)=\theta_{1}, \quad \theta(\phi) \rightarrow \theta_{0} \text { as } \phi \rightarrow \infty
\end{gathered}
$$

has at least one solution $\theta(\phi)$ such that

$$
\operatorname{erfc}\left(\phi / 2 D_{\min }^{\frac{1}{2}}\right) \leq s(\theta(\phi)) \leq \operatorname{erfc}\left(\phi / 2 D_{\max }^{\frac{1}{2}}\right)
$$

for all $\phi$ in $[0, \infty)$.

\section{Proof}

$D(s)$ satisfies a Lipschitz condition in $s$ on any closed subinterval of $[0,1]$ not including the point $s=0$, and in terms of $s$ the boundary value problem is

$$
\begin{gathered}
s^{\prime \prime}+\frac{\phi}{2 D(s)} s^{\prime}=0 \\
s(0)=1, \quad s(\phi) \rightarrow 0 \text { as } \phi \rightarrow \infty .
\end{gathered}
$$

We are required to show that this problem has at least one solution such that

$$
\operatorname{erfc}\left(\phi / 2 D_{\min }^{\frac{1}{2}}\right) \leq s(\phi) \leq \operatorname{erfc}\left(\phi / 2 D_{\max }^{\frac{1}{2}}\right) .
$$


Let $D_{1}, D_{2}$ be numbers such that

$$
0<\mathrm{D}_{1}<\mathrm{D}_{\min }, \quad \mathrm{D}_{\max }<\mathrm{D}_{2}<\infty .
$$

Put $u_{1}(\phi)=\operatorname{erfc}\left(\phi / 2 D_{1}^{\frac{1}{2}}\right), u_{2}(\phi)=\operatorname{erfc}\left(\phi / 2 D_{2}^{\frac{1}{2}}\right)$. Then $u_{1}$ and $u_{2}$ satisfy the linear differential equations

with conditions

$$
u_{i}^{\prime \prime}+\frac{\phi}{2 D_{i}} u_{i}^{\prime}=0 \quad i=1,2
$$

$$
u_{i}(0)=1, \quad u_{i}(\infty)=0, \quad i=1,2 .
$$

Also $u_{1}(\phi)<u_{2}(\phi)$ for finite positive $\phi$. Put

$$
\mathrm{U}=\left\{(\phi, \mathrm{y}): 0 \leq \phi<\infty \text { and } \mathrm{u}_{1}(\phi) \leq \mathrm{y} \leq \mathrm{u}_{2}(\phi)\right\} .
$$

For $b<\infty$, put

$$
\mathrm{U}_{\mathrm{b}}=\left\{(\phi, \mathrm{y}): 0 \leq \phi \leq \mathrm{b} \text { and } \mathrm{u}_{1}(\phi) \leq \mathrm{y} \leq \mathrm{u}_{2}(\phi)\right\} .
$$

If $\mathrm{n}$ is a positive real number, $\mathrm{f}\left(\phi, \mathrm{y}, \mathrm{y}^{\prime}\right)$ is Lipschitzian in the region $U_{b} \times[-n, 0]$, since $U_{b}$ is a subset of $[0, b] \times\left[u_{1}(b), 1\right]$, and $f$ is Lipschitzian in $[0, \mathrm{~b}] \times\left[\mathrm{u}_{1}(\mathrm{~b}), 1\right] \times[-\mathrm{n}, 0]$.

For $r$ a real number, put

$$
v(\phi)=r u_{1}(\phi)+(1-r) .
$$

If we choose $r$ such that $v^{\prime}(0)$ is sufficiently close to $u_{2}^{\prime}(0)$, for instance if

then $\mathrm{v}(\phi)$ satisfies

$$
r=\left(\frac{3 \sqrt{D_{1} / D_{2}}+1}{4}\right),
$$

$$
\begin{gathered}
v^{\prime \prime}+\frac{\phi}{2 D_{1}} v^{\prime}=0 \\
v(0)=1, \quad v(\infty)=1-r, \\
v^{\prime}(0)=-r /\left(\pi D_{1}\right)^{\frac{1}{2}}=m_{2}<u_{2}^{\prime}(0)=-1 /\left(\pi D_{2}\right)^{\frac{1}{2}} .
\end{gathered}
$$

Also $\mathrm{v}(\phi)>\mathrm{u}_{1}(\phi)$ for all $\phi>0$, and there is exactly one $\phi_{1}>0$ such that $v\left(\phi_{1}\right)=u_{2}\left(\phi_{1}\right)$. In other words, $v$ satisfies the same differential equation as $u_{1}, v$ satisfies the initial conditions $v(0)=1$, 
$v^{\prime}(0)=m_{2}<u^{\prime}(0)$, and $v(\phi)$ lies below $u_{2}(\phi)$ for $\phi$ near but not equal to zero, and $v$ crosses $u_{2}$ without meeting $u_{1}$.

Let $y_{2}$ be the solution of

$$
\begin{gathered}
y^{\prime \prime}+\frac{\phi}{2 D(y)} y^{\prime}=0 \\
y(0)=1, \quad y^{\prime}(0)=m_{2} .
\end{gathered}
$$

By the theorem on position of maxima of solutions, we know that $y_{2}$ has the property

$$
\mathrm{m}_{2} \leq \mathrm{y}_{2}^{\prime}(\phi) \leq 0 \text { for } \mathrm{all} \phi \geq 0
$$

Therefore y satisfies the inequality

$$
y_{2}^{\prime \prime}+\frac{\phi}{2 D_{1}} y_{2}^{\prime} \leq 0 \text {. }
$$

We know that $\mathrm{y}_{2}$ satisfies the same initial conditions as $\mathrm{v}$, and $\mathrm{v}$ satisfies

$$
v^{\prime \prime}+\frac{\phi}{2 D_{1}} v^{\prime}=0
$$

Therefore by the comparison theorem, Theorem $6, y_{2}(\phi)$ lies above $v(\phi)$ for all finite $\phi$. Since $v$ crosses $u_{2}$ from below, so must $y_{2}$ cross $u_{2}$ without meeting $u_{1}$. In addition $y_{2}$ satisfies

$$
y_{2}^{\prime \prime}+\frac{\phi}{2 D_{2}} y_{2}^{\prime}>0 \quad \text { for } \phi>0
$$

so the strict inequality together with the relations

$$
\begin{aligned}
& y_{2}(0)=u_{2}(0)=1, \\
& y_{2}^{\prime}(0)<u_{2}(0), \\
& u_{2}^{\prime \prime}+\frac{\phi}{2 D_{2}} u_{2}^{\prime}=0
\end{aligned}
$$

ensures that $\mathrm{y}_{2}(\phi)$ lies strictly below $\mathrm{u}_{2}(\phi)$ for $\phi$ near but not equal to zero, and that $y_{2}(\phi)$ properly crosses $u_{2}(\phi)$ when they meet.

A similar argument demonstrates the existence of $\mathrm{m}$ such that $\mathrm{u}_{1}^{\prime}(0)<\mathrm{m}_{1}<\mathrm{m}_{2}$, and such that there is a function $\mathrm{y}_{1}(\phi)$ satisfying 


$$
\begin{gathered}
y_{1}^{\prime \prime}+\frac{\phi}{2 D\left(y_{1}\right)} y_{1}^{\prime}=0 \\
y_{1}(0)=1, \quad y_{1}^{\prime}(0)=m_{1},
\end{gathered}
$$

and $y_{1}$ properly crosses $u_{1}$ without meeting $u_{2}$.

The topological boundary of the closed set $U_{b}$ is the union of the two sets

and

$$
\left\{(\phi, y):\left(y=u_{1}(\phi) \text { or } y=u_{2}(\phi)\right) \text { and }(0 \leq \phi \leq b)\right\}
$$

$$
\left\{(b, y): u_{1}(b) \leq y \leq u_{2}(b)\right\} .
$$

Let y satisfy (30) with $y(0)=1, y^{\prime}(0)=m$, with $m$ contained in the interval $\left[\mathrm{m}_{1}, \mathrm{~m}_{2}\right]$. By the theorem on position of maxima of solutions, $\mathrm{m} \leq \mathrm{y}^{\prime}(\phi) \leq 0$ for all $\phi$. Since $\mathrm{f}$ satisfies a Lipschitz condition in $\mathrm{U}_{\mathrm{b}} \times\left[\mathrm{m}_{1}, 0\right]$, every solution of $(30)$ with $\mathrm{y}(0)=1, \mathrm{y}^{\prime}(0)=\mathrm{m}, \mathrm{m}_{1} \leq \mathrm{m} \leq 0$, exists at least as far as the boundary of $U_{b}$.

Since y satisfies the inequalities

$$
\begin{array}{ll}
y^{\prime \prime}+\frac{\phi}{2 D_{1}} y^{\prime}<0 & \text { when } \phi>0, \\
y^{\prime \prime}+\frac{\phi}{2 D_{2}} y^{\prime}>0 & \text { when } \phi>0,
\end{array}
$$

the strict inequality ensures that y necessarily crosses the boundary of $\mathrm{U}_{\mathrm{b}}$ at the first point of contact. The strict inequality $u_{1}^{\prime}(0)<\mathrm{m}<\mathrm{u}_{2}^{\prime}(0)$ ensures that $\mathrm{y}(\phi)$ does not touch the boundary of $U_{b}$ when $\phi$ is near but not equal to zero, and for each such $m$ there is a point of the boundary of $U_{b}$ different from $(0,1)$, at which y first touches the boundary of $U_{b}$, and properly crosses the boundary. The Lipschitz conditions ensure that this first point of contact of $y$ with the boundary of $U_{b}$ depends continuously on the parameter $\mathrm{m}$.

In order to prove the existence of a solution to our boundary-value problem we must demonstrate the existence of $\mathrm{m}$ contained in $\left[\mathrm{m}_{1}, \mathrm{~m}_{2}\right]$ such that there is a function $y(\phi)$ satisfying (30) and 


$$
\begin{gathered}
y(0)=1, \quad y^{\prime}(0)=m, \\
u_{1}(\phi) \leq y(\phi) \leq u_{2}(\phi) \quad \text { for all } \phi \geq 0, \\
\underset{\substack{\operatorname{limit} \\
\phi \rightarrow \infty}}{ }[y(\phi)]=0 .
\end{gathered}
$$

Suppose that no such $m$ exists. Then for each $m$ in $\left[m_{1}, m_{2}\right]$, we have a solution $y(\phi)$ of (30) and (31) which exists as far as the boundary of $\mathrm{U}_{\mathrm{b}}$ for $\mathrm{all} \mathrm{b}<\infty$. Hence each such $\mathrm{y}$ exists as far as the boundary of $\mathrm{U}$, and must cross the boundary of $U$ at the first point of contact. The supposition that no m exists satisfying all of (31), (32) and (33) implies the existence of such a point of contact of y with the boundary of $U$. This point of contact is on the boundary of $a U_{b}$, for some $b<\infty$, Accordingly we can define a continuous mapping from $\left[\mathrm{m}_{1}, \mathrm{~m}_{2}\right]$ into that part of the boundary of $U$ which does not contain the point $(0,1)$. This mapping $\mathrm{T}$ is well-defined for all $\mathrm{m}$ in $\left[\mathrm{m}_{1}, \mathrm{~m}_{2}\right]$, if we 1 et $\mathrm{T}(\mathrm{m})$ be the first point of contact of $y(\phi)$ with the boundary of $U$, in the sense of the previous paragraph.

We have $T$ mapping the interval $\left[\mathrm{m}_{1}, \mathrm{~m}_{2}\right]$ into the set $G_{1} \cup \mathrm{G}_{2}$, where

$$
G_{i}=\left\{(\phi, y): \phi>0 \text { and } u_{i}(\phi)=y\right\}, \quad i=1,2 .
$$

Hence $T$ maps the closed interval $\left[\mathrm{m}_{1}, \mathrm{~m}_{2}\right]$ into the set $G_{1} \cup G_{2}$, such that $T\left(m_{1}\right)$ is in $G_{1}$ which is an open subset of $G_{1} \cup G_{2}$, for $i=1,2$. $G_{1}$ and $G_{2}$ are disjoint. In other words, we have a continuous mapping of the connected set $\left[m_{1}, m_{2}\right]$ into the two components of the set $G_{1} \cup G_{2}$, which is a contradiction.

Therefore there exists $\mathrm{m} \in\left[\mathrm{m}_{1}, \mathrm{~m}_{2}\right]$ such that $\mathrm{s}(\phi)$ satisfies

$$
\mathrm{s}(0)=1, \quad \mathrm{~s}^{\prime}(0)=\mathrm{m}, \quad \mathrm{s}(\infty)=0,
$$

and

$$
\operatorname{erfc}\left(\phi / 2 D_{1}^{\frac{1}{2}}\right) \leq s(\phi) \leq \operatorname{erfc}\left(\phi / 2 D_{2}^{\frac{1}{2}}\right) .
$$

Inequality (34) is true for all $D_{1}, D_{2}$ such that 


$$
0<\mathrm{D}_{1}<\mathrm{D}_{\min }, \quad \mathrm{D}_{\max }<\mathrm{D}_{2}<\infty .
$$

The strict inequality in (35) can be relaxed as follows. For all $\phi \geq 0$, and

$$
\operatorname{erfc}\left(\phi / 2 D_{\min }^{\frac{1}{2}}\right)=\sup _{D_{1}<D_{\min }}\left[\operatorname{erfc}\left(\phi / 2 D_{1}^{\frac{1}{2}}\right)\right]
$$

Therefore

$$
\operatorname{erfc}\left(\phi / 2 D_{\max }^{\frac{1}{2}}\right)=\inf _{D_{2}>D_{\max }}\left[\operatorname{erfc}\left(\phi / 2 D_{2}^{\frac{1}{2}}\right)\right]
$$

$$
\operatorname{erfc}\left(\phi / 2 D_{\min }^{\frac{1}{2}}\right) \leq s(\phi) \leq \operatorname{erfc}\left(\phi / 2 D_{\max }^{\frac{1}{2}}\right)
$$

as required.

When the function $D(\theta)$ is discontinuous, so is $D(\theta(s))$, or $D(s)$. At a point of discontinuity in $D(s)$ we do not in general have a solution of

$$
\begin{aligned}
& s^{\prime \prime}+\frac{\phi}{2 D(s)} s^{\prime}=0 \\
& s(0)=1, \quad s(\infty)=0,
\end{aligned}
$$

where by a "solution" we mean a function which is a twice continuously differentiable function of $\phi$. However we can obtain a physically meaningful answer if we allow $s^{\prime \prime}$ to be discontinuous (and undefined) at a value of $\phi$ which corresponds to a discontinuity of $\mathrm{D}(\mathrm{s}(\phi))$. We require both the function $s$ and its derivative $s^{\prime}$ to be continuous for all $\phi$. In terms of the variable $\theta$, this is continuity of $\theta$ and of $D(\theta) \frac{d \theta}{d \phi}$. The latter "matching condition" corresponds to continuity of flux, which is necessary on physical grounds.

If $D(\theta)$ is a piecewise continuous function on $\left[\theta_{0}, \theta_{1}\right]$, then $D(\theta)$ has a finite number of points of jump discontinuity $\theta_{2}, \theta_{3}, \ldots, \theta_{n}$. At each such $\theta_{\mathrm{k}}$ the two limits

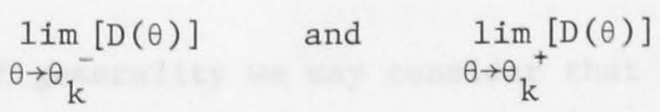

exist and are different, and $D(\theta)$ is continuous and bounded on each open interval $\left(\theta_{0}, \theta_{2}\right),\left(\theta_{2}, \theta_{3}\right), \ldots$ Therefore $\mathrm{D}(\theta)$ may be extended to a 
continuous function on each of the closed intervals $\left[\theta_{0}, \theta_{2}\right],\left[\theta_{2}, \theta_{3}\right], \ldots$

If in addition $D(\theta)$ satisfies a Lipschitz condition on each interval $\left(\theta_{0}, \theta_{2}\right),\left(\theta_{2}, \theta_{3}\right), \ldots$ and $D(\theta)$ is bounded away from zero on $\left[\theta_{0}, \theta_{1}\right]$, then we may extend our existence theorem to solutions of this type. We again take $\theta_{0}<\theta_{1}$.

Theorem 11 Existence for Piecewise Continuous $D(\theta)$.

Let $D(\theta)$ be defined and piecewise continuous on $\left[\theta_{0}, \theta_{1}\right]$, and such that $\underset{\theta}{\inf }[D(\theta)]=D_{\min }>0$, and $\sup _{\theta}[D(\theta)]=D_{\max }<\infty$. Let the finite set of points of jump discontinuity of $D$ be $\theta_{2}, \theta_{3}, \ldots, \theta_{n}$, with $\theta_{0}<\theta_{2}<\theta_{3} \ldots<\theta_{n}<\theta_{1}$. Let $D(\theta)$ satisfy a Lipschitz condition on each interval $\left[\theta_{0}, \theta_{2}\right),\left(\theta_{2}, \theta_{3}\right), \ldots\left(\theta_{n}, \theta_{1}\right]$. Then there exist points $\phi_{2}, \phi_{3}, \ldots, \phi_{n}$, with $0<\phi_{n}<\ldots<\phi_{3}<\phi_{2}<\infty$, such that

$$
\frac{d}{d \phi}\left(D(\theta) \frac{d \theta}{d \phi}\right)+\frac{\phi}{2} \frac{d \theta}{d \phi}=0
$$

for al1 $\phi \geq 0$ except $\phi_{2}, \phi_{3}, \ldots, \phi_{n}$,

$$
\begin{aligned}
& \theta(0)=1, \quad \underset{\phi \rightarrow \infty}{\operatorname{limit}} \theta(\phi)=0 \\
& \theta\left(\phi_{k}\right)=\theta_{k}, \quad k=2, \ldots, n
\end{aligned}
$$

and

Put

$$
\operatorname{limit}_{\phi \rightarrow \phi_{k}^{-}}\left[D(\theta) \frac{d \theta}{d \phi}\right]=\operatorname{limit}_{\phi \rightarrow \phi_{k}^{+}}\left[D(\theta) \frac{d \theta}{d \phi}\right] \quad k=2, \ldots, n \text {. }
$$

Then for a $11 \phi \geq 0$,

$$
s(\theta)=\frac{\int_{\theta_{0}}^{\theta} D(a) d a}{\int_{\theta_{0}}^{\theta_{1}} D(a) d a} .
$$

$$
\operatorname{erfc}\left(\phi / 2 D_{\min }^{\frac{1}{2}}\right) \leq s(\theta(\phi)) \leq \operatorname{erfc}\left(\phi / 2 D_{\max }^{\frac{1}{2}}\right) .
$$

\section{Proof}

Without loss of generality we may consider that $D(\theta)$ has only one point of jump discontinuity, $\theta_{2}$. On applying the Kirchhoff transformation we find that the function $\mathrm{D}(\mathrm{s})$ has a jump discontinuity at $s_{2}$, and satisfies a Lipschitz condition on each of the intervals $\left[0, s_{2}\right)$ and 
$\left(s_{2}, 1\right]$. If we divide the $s-\phi$ plane at the line $s=s_{2}$, and define the function $f\left(\phi, s, s^{\prime}\right)$ by

$$
f\left(\phi, s, s^{\prime}\right)=\frac{\phi}{2 D(s)} s^{\prime}
$$

then $f$ can be extended to a function $f_{1}$ which is continuous and Lipschitzian in the region $H_{1}=[0, b] \times\left[0, s_{2}\right] \times[-n, 0]$, and can be extended to a function $f_{2}$ which is continuous and Lipschitzian in the region $\mathrm{H}_{2}=[0, \mathrm{~b}] \times\left[\mathrm{s}_{2}, 1\right] \times[-\mathrm{n}, 0]$. If $\mathrm{y}$ is a solution of

$$
\begin{gathered}
y^{\prime \prime}+f_{2}\left(\phi, y, y^{\prime}\right)=0 \\
y(0)=1, \quad y^{\prime}(0)=m, \quad-n \leq m \leq 0,
\end{gathered}
$$

then $y$ can be uniquely continued as far as the boundary of $\mathrm{H}_{2}$. If $y$ meets the line $y=s_{2}$, then we have $c$ such that $0<c \leq b, y(c)=s_{2}$, and $\mathrm{y}^{\prime}(\mathrm{c})=\mathrm{h}, \mathrm{m}<\mathrm{h}<0$. The point $(\mathrm{c}, \mathrm{h})$ depends continuously on the parameter $\mathrm{m}$.

In $H_{1}$ we can define a new initial value problem by

$$
\begin{gathered}
y^{\prime \prime}+f_{1}\left(\phi, y, y^{\prime}\right)=0 \\
y(c)=s_{2}, \quad y^{\prime}(c)=h .
\end{gathered}
$$

The solution y can be uniquely continued as far as the boundary of $\mathrm{H}_{1}$, and it depends continuously on the quantities $c$ and $h$ by Theorem 4 . Therefore the solution y depends continuously on the parameter $m$ in the region $H_{1}$. We have shown how to construct a function y satisfying

$$
\begin{gathered}
y(0)=1, \quad y^{\prime}(0)=m \\
y^{\prime \prime}+\frac{\phi}{2 D(y)} y^{\prime}=0
\end{gathered}
$$

for all $\phi \geq 0$ except for (possibly) one $\phi_{2}$ such that

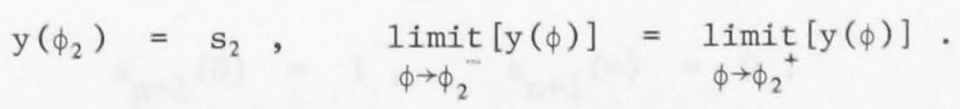

Also $y$ depends continuously on the parameter $m$ in the region $\mathrm{H}_{1} \cup \mathrm{H}_{2}$.

The rest of the proof follows the same lines as that of Theorem 10 . 


\subsection{THE METHOD.}

It may be easily verified that for a suitable well-behaved function $D(s)$ the differential equation

$$
s^{\prime \prime}+\frac{\phi}{2 D(s)} s^{\prime}=0
$$

with conditions $s(0)=1, s(\infty)=0$ is equivalent to an integral equation

$$
s(\phi)=\frac{\int_{\phi}^{\infty} \exp \left(-\int_{0}^{n} \frac{\mathrm{ada}}{2 \mathrm{D}(\mathrm{s}(\mathrm{a}))}\right) \mathrm{d} n}{\int_{0}^{\infty} \exp \left(-\int_{0}^{n} \frac{\mathrm{ada}}{2 \mathrm{D}(\mathrm{s}(\mathrm{a}))}\right) \mathrm{dn}} .
$$

Crank and Henry (1949a, 1949b) constructed an iterative scheme to solve (38) by the method of successive approximations. In (38) they put

$$
s_{n+1}(\phi)=\frac{\int_{\phi}^{\infty} \exp \left(-\int_{0}^{n} \frac{a d a}{2 D\left(s_{n}(a)\right)}\right) d n}{\int_{0}^{\infty} \exp \left(-\int_{0}^{n} \frac{a d a}{2 D\left(s_{n}(a)\right)}\right) d n} .
$$

If $s_{n}(\phi)$ is an estimate of the solution of (38), then hopefully $s_{n+1}(\phi)$ is a "better" estimate. In order to start the iteration scheme, a suitable starting function $s_{1}(\phi)$ must be guessed.

Determining the successive functions $s_{n+1}$ from (39) is equivalent to solving a succession of linear problems

$$
\begin{gathered}
\frac{d^{2} s_{n+1}}{d \phi^{2}}+\frac{\phi}{2 D\left(s_{n}(\phi)\right)} \frac{d s_{n+1}}{d \phi}=0 \\
s_{n+1}(0)=1, \quad s_{n+1}(\infty)=0 .
\end{gathered}
$$

Consequently it will prove fruitful to study the properties of such linear equations. Let $D(s)$ be a non-decreasing function on $[0,1]$. We 
would like to show that this monotone property of $D$ results in certain monotone properties of successive solutions of (39).

\subsection{PRELIMINARIES}

We would like to be able to compare solutions of two different equations (39), when the coefficient of $s^{\prime}$ in one equation is greater than that in the other. Statements about strict inequality can be proved, and then relaxed to inequality. The following theorem extends results of Bailey, Shampine and Waltman (1968), Chapter 5, about boundary value problems on the finite interval, to the semi-infinite interval for our particular problem.

Theorem 12 Comparison Lemma for Solutions to Linear Boundary Value Problems.

Let $g_{1}, g_{2}$ be functions of $\phi$ continuous on $[0, \infty)$, with two constants $\mathrm{h}, \mathrm{k}>0$, such that

$$
h \geq g_{i}(\phi) \geq k \text { for all } \phi \geq 0, \quad i=1,2 \text {. }
$$

Let $\mathrm{y}_{1}, \mathrm{y}_{2}$ be defined and continuous with continuous derivatives of first and second order on $[0, \infty)$, such that

and

$$
y_{i}^{\prime \prime}+\frac{\phi}{2 g_{i}(\phi)} y_{i}^{\prime}=0
$$

$$
y_{i}(0)=1, \quad y_{i}(\infty)=0, \quad \text { for } i=1,2 \text {. }
$$

When

$$
g_{1}(\phi)<g_{2}(\phi) \quad \text { for all } \phi>0 \text {, }
$$

then

$$
\mathrm{y}_{1}(\phi)<\mathrm{y}_{2}(\phi) \quad \text { for a } 11 \phi>0 \text {. }
$$

And when

$$
g_{1}(\phi) \leq g_{2}(\phi) \quad \text { for all } \phi \geq 0 \text {, }
$$

then

$$
\mathrm{y}_{1}(\phi) \leq \mathrm{y}_{2}(\phi) \quad \text { for } \mathrm{a} 11 \phi \geq 0 \text {. }
$$




\section{$\underline{\text { Proof }}$}

The strict inequality will be proved first.

Assume that $g_{1}(\phi)<g_{2}(\phi)$ for all $\phi>0$. From the proof of Theorem 8 , we have explicit expressions for $y_{i}^{\prime}, i=1,2$.

$$
y_{i}^{\prime}(0)=-\frac{1}{\int_{0}^{\infty} \exp \left(-\int_{0}^{n} \frac{a d a}{2 g_{i}(a)}\right) d n} \quad i=1,2 \text {. }
$$

Therefore $y_{1}^{\prime}(0)<y_{2}^{\prime}(0)$, so $y_{1}(\phi)<y_{2}(\phi)$ for $\phi$ near but not equal to zero.

Suppose that the conclusion of the theorem that $y_{1}(\phi)<y_{2}(\phi)$ for all $\phi>0$ is not true. Then there is at least one $\phi>0$ such that $y_{1}(\phi)=y_{2}(\phi)$.

The set of all such $\phi>0$ is a closed subset of $(0, \infty)$, whose complement in $[0, \infty)$ contains a neighbourhood of zero. Therefore there is a smallest $\phi_{1}$ such that

$$
\mathrm{y}_{1}\left(\phi_{1}\right)-\mathrm{y}_{2}\left(\phi_{1}\right)=0 \text {, and } \phi_{1}>0 \text {. }
$$

We now prove that $y_{1}$ properly crosses $y_{2}$ at $\phi_{1}$. For $\phi$ near $\phi_{1}$ but less than $\phi_{1}$,

$$
y_{1}(\phi)-y_{2}(\phi)<0 .
$$

Therefore for such $\phi$,

Now

$$
y_{1}^{\prime}(\phi)-y_{2}^{\prime}(\phi) \geq 0
$$

Suppose that

$$
y_{1}^{\prime \prime}(\phi)-y_{2}^{\prime \prime}(\phi)=-\frac{\phi}{2 g_{1}(\phi)} y_{1}^{\prime}+\frac{\phi}{2 g_{2}(\phi)} y_{2}^{\prime} .
$$

Then

$$
\mathrm{y}_{1}^{\prime}\left(\phi_{1}\right)-\mathrm{y}_{2}^{\prime}\left(\phi_{1}\right)=0 \text {. }
$$

We know that

$$
\left(y_{1}-y_{2}\right)^{\prime \prime}\left(\phi_{1}\right)=-\frac{\phi_{1} y_{1}^{\prime}}{2}\left(\frac{1}{g_{1}\left(\phi_{1}\right)}-\frac{1}{g_{2}\left(\phi_{1}\right)}\right) \text {. }
$$

Therefore

$$
\phi_{1}>0, \quad y_{1}^{\prime}<0, \text { and } \frac{1}{g_{1}\left(\phi_{1}\right)}>\frac{1}{g_{2}\left(\phi_{1}\right)} \text {. }
$$

$$
\left(y_{1}-y_{2}\right)^{\prime \prime}\left(\phi_{1}\right)>0
$$

We now have the situation that $\left(y_{1}-y_{2}\right)^{\prime \prime}\left(\phi_{1}\right)>0,\left(y_{1}-y_{2}\right)^{\prime}\left(\phi_{1}\right)=0$, and $\left(y_{1}-y_{2}\right)^{\prime}(\phi)>0$ when $\phi$ is near but less than $\phi_{1}$, which is impossible. 
This contradiction means that in fact $\left(y_{1}-y_{2}\right)^{\prime}\left(\phi_{1}\right)=m>0$. In other words, $y_{1}$ and $y_{2}$ cannot agree in both value and slope at $\phi_{1}$, and so $y_{1}$ properly crosses $y_{2}$ at $\phi_{1}$.

Let $\mathrm{v}(\phi)$ be the function which satisfies the same initial conditions as $\mathrm{y}_{1}$ at $\phi_{1}$, namely

$$
\mathrm{v}\left(\phi_{1}\right)=\mathrm{y}_{1}\left(\phi_{1}\right), \quad \mathrm{v}^{\prime}\left(\phi_{1}\right)=\mathrm{y}_{1}^{\prime}\left(\phi_{1}\right),
$$

and the same equation as $y_{2}$, namely

$$
v^{\prime \prime}+\frac{\phi}{2 g_{2}(\phi)} v^{\prime}=0
$$

Then the function $\left(v-y_{2}\right)$ satisfies

$$
\begin{gathered}
\left(v-y_{2}\right)^{\prime \prime}+\frac{\phi}{2 g_{2}(\phi)}\left(v-y_{2}\right)^{\prime}=0, \\
\left(v-y_{2}\right)\left(\phi_{1}\right)=0, \quad\left(v-y_{2}\right)^{\prime}\left(\phi_{1}\right)=m>0 .
\end{gathered}
$$

Explicitly,

$$
\left(v-y_{2}\right)(\phi)=m \int_{\phi_{1}}^{\phi} \exp \left(-\int_{\phi_{1}}^{n} \frac{a d a}{2 g_{2}(a)}\right) d n \quad \text { when } \phi \geq \phi_{1} \text {. }
$$

This shows that for all $\phi>\phi_{1}, v(\phi)>y_{2}(\phi)$, and also that $v(\infty)>y_{2}(\infty)$.

Now we can compare $y_{1}$ and $v$, which satisfy respectively

and

$$
y_{1}^{\prime \prime}+\frac{\phi}{2 g_{1}(\phi)} y_{1}^{\prime}=0
$$

Therefore

$$
v^{\prime \prime}+\frac{\phi}{2 g_{2}(\phi)} v^{\prime}=0
$$

$$
v^{\prime \prime}+\frac{\phi}{2 g_{1}(\phi)} v^{\prime} \leq 0 \text { for all } \phi \geq \phi_{1} \text {. }
$$

Also $v$ and $y_{1}$ satisfy the same initial conditions at $\phi=\phi_{1}$. The Comparison Theorem, Theorem 6 , shows that

$$
y_{1}(\phi) \geq v(\phi) \quad \text { for all } \phi \geq \phi_{1} \text {. }
$$

Therefore

$$
\underset{\phi \rightarrow \infty}{\operatorname{limit}} \mathrm{y}_{1}(\phi) \geq \underset{\phi \rightarrow \infty}{\operatorname{limit}} \mathrm{v}(\phi)>\underset{\phi \rightarrow \infty}{\operatorname{limit}} \mathrm{y}_{2}(\phi) .
$$

This is a contradiction to the hypothesis that

$$
\underset{\phi \rightarrow \infty}{\operatorname{limit}} y_{1}(\phi)=\underset{\phi \rightarrow \infty}{\operatorname{limit}} y_{2}(\phi)=0
$$


Therefore no such $\phi_{1}$ exists, and so for all $\phi>0, y_{1}(\phi)<y_{2}(\phi)$.

The first statement of the theorem is now proved, and can be used to prove the second statement.

Let $g_{1}, g_{2}$ be such that $g_{1}(\phi) \leq g_{2}(\phi)$ for all $\phi \geq 0$. Put $g_{3}(\phi)=g_{2}(\phi)+\varepsilon$, where $\varepsilon$ is a small positive number.

Let $\mathrm{y}_{1}, \mathrm{y}_{2}, \mathrm{y}_{3}$ be twice continuously differentiable functions satisfying

$$
\begin{gathered}
y_{i}^{\prime \prime}+\frac{\phi}{2 g_{i}(\phi)} y_{i}^{\prime}=0, \\
y_{i}(0)=1, \quad y_{i}(\infty)=0 \quad \text { for } i=1,2,3 .
\end{gathered}
$$

Then $g_{1}(\phi)<g_{3}(\phi)$, and $g_{2}(\phi)<g_{3}(\phi)$, for all $\phi>0$. This strict inequality ensures that

$$
y_{2}(\phi)<y_{3}(\phi) \text {, and } y_{2}(\phi)<y_{3}(\phi), \quad \text { for all } \phi>0 \text {. }
$$

The existence of $h, k$ such that $h \geq g_{3}(\phi) \geq k$ means that as $\varepsilon \rightarrow 0$ and $g_{3}(\phi) \rightarrow g_{2}(\phi)$ for each $\phi \geq 0, y_{3}(\phi) \rightarrow y_{2}(\phi)$ for each $\phi \geq 0$. Therefore $y_{1}(\phi) \leq y_{2}(\phi)$, for a11 $\phi \geq 0$, and so the second statement of the theorem is proved.

\subsection{CONVERGENCE PROOF FOR NON-DECREASING D}

The comparison lemma, Theorem 12, will enable us to prove the convergence of the method of Crank and Henry for non-decreasing $D(s)$ when D is sufficiently well behaved, and a suitable starting function is used. The convergence of the iteration procedure to a solution of (37) for a continuous, positive, non-decreasing $\mathrm{D}(\mathrm{s})$ gives an improvement of the existence theorem, Theorem 11, for such $D(s)$ functions. In terms of $\theta$, the non-decreasing property for positive continuous $D(s)$ is equivalent to $D(\theta)$ being a non-decreasing function of $\theta$ on $\left[\theta_{0}, \theta_{1}\right]$, when $\theta_{0}<\theta_{1}$. This is the "absorption" case. 
When $D$ also has properties sufficient to ensure the uniqueness of such a solution to the problem, it will be shown that the iteration procedure converges to the unique solution with any suitable starting function. By Seyferth's uniqueness theorem, the continuity and positivity of $\mathrm{D}(\theta)$ on $\left[\theta_{0}, \theta_{1}\right]$ and the existence of a continuous derivative $d D / d \theta$ is sufficient to ensure uniqueness.

Theorem 14 Convergence for Non-decreasing D(s).

Let $D(s)$ be a continuous function of $s$ defined on $[0,1]$, such that $D(s)$ is non-decreasing in $\mathrm{s}$ and $\mathrm{D}_{\min }=\mathrm{D}(0)>0$. Then the sequence of functions defined by

$$
\begin{aligned}
s_{0}(\phi) & =1 \quad \text { for a } 11 \phi \geq 0 \\
s_{n+1}(\phi) & =\frac{\int_{\phi}^{\infty} \exp \left(-\int_{0}^{n} \frac{a d a}{2 D\left(s_{n}(a)\right)}\right) d n}{\int_{0}^{\infty} \exp \left(-\int_{0}^{n} \frac{a d a}{2 D\left(s_{n}(a)\right)}\right) d n}
\end{aligned}
$$

converges to a function $s(\phi)$ satisfying

and

$$
s^{\prime \prime}+\frac{\phi}{2 D(s)} s^{\prime}=0 \quad \text { for } a 11 \phi \geq 0
$$

$$
s(0)=1, \quad s(\infty)=0 .
$$

If in addition $D(s)$ satisfies conditions sufficient for uniqueness of a solution of (42) then the sequence of functions (41) converges with any continuous starting function $s_{0}(\phi)$ satisfying $0 \leq s_{0}(\phi) \leq 1$ for all $\phi \geq 0$, to the unique solution of (42) and (43).

\section{Proof}

Let $s_{0}$ be defined by $s_{0}(\phi)=1$ for all $\phi \geq 0$. Suppose we have continuous functions $u_{1}(\phi), u_{2}(\phi)$, such that

$$
0 \leq \mathrm{u}_{1}(\phi) \leq \mathrm{u}_{2}(\phi) \leq 1 \text {, for all } \phi \geq 0 \text {. }
$$

Then

$$
D(0) \leq D\left(u_{1}(\phi)\right) \leq D\left(u_{2}(\phi)\right) \leq D(1), \text { for a } 11 \phi \geq 0 .
$$


We define the operator $\mathrm{T}$ on the function $\mathrm{u}$ by

$$
(\mathrm{Tu})(\phi)=\frac{\int_{\phi}^{\infty} \exp \left(-\int_{0}^{n} \frac{\mathrm{ada}}{2 \mathrm{D}(\mathrm{u}(\mathrm{a}))}\right) \mathrm{d} n}{\int_{0}^{\infty} \exp \left(-\int_{0}^{n} \frac{\mathrm{ada}}{2 \mathrm{D}(\mathrm{u}(\mathrm{a}))}\right) \mathrm{d} n} .
$$

The function $(\mathrm{Tu})(\phi)$ satisfies the linear equation

and

$$
(\mathrm{Tu})^{\prime \prime}+\frac{\phi}{2 \mathrm{D}(\mathrm{u}(\phi))}(\mathrm{Tu})^{\prime}=0 \text {, }
$$

$$
(\mathrm{Tu})(0)=1, \quad(\mathrm{Tu})(\infty)=0 .
$$

Application of the comparison lemma, Theorem 13, to (45) using (44) shows that for all $\phi \geq 0$,

$$
\operatorname{erfc}\left(\frac{\phi}{2[\mathrm{D}(0)]^{\frac{1}{2}}}\right) \leq\left(\mathrm{Tu}_{1}\right)(\phi) \leq\left(\mathrm{Tu}_{2}\right)(\phi) \leq \operatorname{erfc}\left(\frac{\phi}{2[\mathrm{D}(1)]^{\frac{1}{2}}}\right)=\left(\mathrm{Ts}_{0}\right)(\phi) \cdot(46)
$$

We can write $s_{1}=T s_{0}, s_{2}=T s_{1}=T^{2} s_{0}$, etc. Then we have for all $\phi \geq 0$

$$
\operatorname{erfc}\left(\frac{\phi}{2[D(0)]^{\frac{1}{2}}}\right) \leq s_{2}(\phi) \leq s_{1}(\phi)=\operatorname{erfc}\left(\frac{\phi}{2[D(i)]^{\frac{1}{2}}}\right) \text {. }
$$

Induction on $\mathrm{n}$ shows that for all $\mathrm{n}>0$ and $\mathrm{all} \phi \geq 0$,

$$
\operatorname{erfc}\left(\frac{\phi}{2[D(0)]^{\frac{1}{2}}}\right) \leq s_{n+1}(\phi) \leq s_{n}(\phi) \leq s_{1}(\phi) \text {. }
$$

Therefore the sequence $\left\{s_{n}\right\}$ is a decreasing sequence of continuous functions, bounded below. For each n,

$$
s_{n}^{\prime}(0) \leq s_{n}^{\prime}(\phi) \leq 0 \quad \text { for all } \phi \geq 0 \text {. }
$$

For a $11 \mathrm{n} \geq 0$,

$$
-\infty<-1 /[\pi \mathrm{D}(0)]^{\frac{1}{2}} \leq s_{\mathrm{n}}^{\prime}(0) \leq-1 /[\pi \mathrm{D}(1)]^{\frac{1}{2}} .
$$

Therefore for all $\mathrm{n} \geq 0$ and for all $\phi_{1}, \phi \geq 0$,

$$
\left|s_{n}(\phi)-s_{n}\left(\phi_{1}\right)\right|<\frac{\left|\phi-\phi_{1}\right|}{[D(0)]^{\frac{1}{2}}} \text {. }
$$

The right hand side of inequality (47) is independent of $\mathrm{n}$. Therefore $\left\{s_{n}\right\}$ is an equicontinuous sequence of functions. On any interval [0,b] the sequence $\left\{s_{n}\right\}$ is a bounded sequence of equicontinuous functions. By the theorem of Arzelà-Ascoli (see for example Rudin, 1964, p.144), the sequence $\left\{s_{n}\right\}$ has a subsequence $\left\{s_{n_{k}}\right\}$ which is uniformly convergent on 
the interval $[0, \mathrm{~b}]$ to a continuous function.

Because the sequence $\left\{s_{n}\right\}$ is a decreasing sequence, all such subsequences of $\left\{s_{n}\right\}$, and $\left\{s_{n}\right\}$ itself, converge to the same continuous limit function $s(\phi)$ on $[0, b]$. This is true for any $b<\infty$, so $\left\{s_{n}\right\}$ converges to a continuous limit function s on $[0, \infty)$, the convergence being uniform on finite intervals.

Continuity of $\mathrm{D}(\mathrm{s})$ means that for all finite $\phi$,

$$
\operatorname{limit}_{\mathrm{n} \rightarrow \infty} \frac{\phi}{2 \mathrm{D}\left(\mathrm{s}_{\mathrm{n}}(\phi)\right)}=\frac{\phi}{2 \mathrm{D}(\mathrm{s}(\phi))} .
$$

We know that for all finite $\phi$ and all $\mathrm{n} \geq 0$,

$$
\frac{\phi}{2 \mathrm{D}\left(\mathrm{s}_{\mathrm{n}}(\phi)\right)} \leq \frac{\phi}{2 \mathrm{D}(0)}
$$

By the Lebesgue Dominated Convergence Theorem (see for example Williamson, 1962, p.60), for all finite $\phi$

By continuity

$$
\underset{n \rightarrow \infty}{\operatorname{limit}}\left[\int_{0}^{\phi} \frac{a d a}{2 D\left(s_{n}(a)\right)}\right]=\int_{0}^{\phi} \frac{a d a}{2 D(s(a))} .
$$

$$
\operatorname{limit}_{n \rightarrow \infty}\left[\exp \left(-\int_{0}^{\phi} \frac{\mathrm{ada}}{2 \mathrm{D}\left(\mathrm{s}_{\mathrm{n}}(\mathrm{a})\right)}\right)\right]=\exp \left(-\int_{0}^{\phi} \frac{\mathrm{ada}}{2 \mathrm{D}(\mathrm{s}(\mathrm{a}))}\right) .
$$

For all $\mathrm{n} \geq 0$ and all $\phi \geq 0$,

$$
\exp \left(-\int_{0}^{\phi} \frac{a d a}{2 D\left(s_{n}(a)\right)}\right) \leq \exp \left(-\frac{\phi^{2}}{4 D(1)}\right) \text {. }
$$

By the Lebesgue Dominated Convergence Theorem, for all $\phi \geq 0$

$$
\operatorname{limit}_{n \rightarrow \infty}\left[\int_{\phi}^{\infty} \exp \left(-\int_{0}^{n} \frac{\mathrm{ada}}{2 \mathrm{D}\left(\mathrm{s}_{\mathrm{n}}(\mathrm{a})\right)}\right) \mathrm{d} n\right]=\int_{\phi}^{\infty} \exp \left(-\int_{0}^{n} \frac{\mathrm{ada}}{2 \mathrm{D}(\mathrm{s}(\mathrm{a}))}\right) \mathrm{d} n \text {. }
$$

Therefore for all $\phi \geq 0$,

$$
\begin{aligned}
s(\phi) & =\operatorname{limit}_{n \rightarrow \infty}\left[s_{n}(\phi)\right] \\
& =\operatorname{limit}_{n \rightarrow \infty}\left[\frac{\left.\int_{\phi}^{\infty} \exp \left(-\int_{0}^{n} \frac{a d a}{2 D\left(s_{n}(a)\right)}\right) d n\right]}{\int_{0}^{\infty} \exp \left(-\int_{0}^{n} \frac{a d a}{2 D\left(s_{n}(a)\right)}\right) d n}\right]=\frac{\int_{\phi}^{\infty} \exp \left(-\int_{0}^{n} \frac{a d a}{2 D(s(a))}\right) d n}{\int_{0}^{\infty} \exp \left(-\int_{0}^{n} \frac{a d a}{2 D(s(a))}\right) d n} .
\end{aligned}
$$


We have shown that s satisfies the integral equation (38). Two applications of the Fundamental Theorem of Calculus show that s satisfies equation (42) and conditions (43) as required, and the first part of the theorem is proved.

We may similarly prove that with starting function $v_{0}$ such that $v_{0}(\phi)=0$ for all $\phi \geq 0$, an increasing sequence $\left\{v_{n}\right\}$ of equicontinuous functions is obtained, bounded above. The sequence $\left\{\mathrm{v}_{\mathrm{n}}\right\}$ converges to a continuous function $v$ which is a solution of (42) and (43). Repeated application of (46) shows that if $\mathrm{w}$ is another solution to (42) and (43), then for all $\phi \geq 0$ and all $n \geq 0$

Therefore

$$
v_{n}(\phi) \leq w(\phi) \leq s_{n}(\phi) .
$$

$$
v(\phi) \leq w(\phi) \leq s(\phi),
$$

and so $\mathrm{v}$ and $\mathrm{s}$ are respectively minimal and maximal solutions to (42) and (43).

If $D$ also satisfies conditions sufficient to ensure uniqueness of a solution of (42) and (43), such as the existence of a continuous derivative

$$
\frac{d D}{d \theta}=\frac{d D}{d s} \cdot \frac{d s}{d \theta}=\frac{d D}{d s} \cdot \frac{D(\theta)}{\int_{\theta_{0}}^{\theta_{1}} D(a) d s}
$$

then $v(\phi)=s(\phi)$ for all $\phi \geq 0$.

In the case that uniqueness applies, let $w_{0}$ be defined and continuous on $[0, \infty)$, such that

$$
0 \leq w_{0}(\phi) \leq 1 \quad \text { for all } \phi \geq 0 \text {. }
$$

That is,

$$
\mathrm{v}_{0}(\phi) \leq \mathrm{w}_{0}(\phi) \leq \mathrm{s}_{0}(\phi) \text { for a11 } \phi \geq 0 \text {. }
$$

Therefore all $\phi \geq 0$,

$$
v_{1}(\phi)=\left(\operatorname{Tv}_{0}\right)(\phi) \leq\left(\operatorname{Tw}_{0}\right)(\phi) \leq\left(\mathrm{Ts}_{0}\right)(\phi)=\mathrm{s}_{1}(\phi) .
$$

By induction, for all $\mathrm{n} \geq 0$ and all $\phi \geq 0$, 


$$
v_{n}(\phi) \leq w_{n}(\phi) \leq s_{n}(\phi)
$$

Therefore $w(\phi)=\underset{n \rightarrow \infty}{\operatorname{limit}}\left[w_{n}(\phi)\right]$ exists for all $\phi \geq 0$, and

$$
\begin{gathered}
\underset{n \rightarrow \infty}{\operatorname{limit}}\left[v_{n}(\phi)\right] \leq \underset{n \rightarrow \infty}{\operatorname{limit}}\left[w_{n}(\phi)\right] \leq \underset{n \rightarrow \infty}{\operatorname{imit}}\left[s_{n}(\phi)\right] \\
v(\phi)=w(\phi)=s(\phi) .
\end{gathered}
$$

Therefore if $D(s)$ satisfies conditions sufficient to ensure uniqueness, then the iteration procedure of Crank and Henry converges to the solution of (42) and (43) for any such $w_{0}$.

In practice the quadratures in (41) must be performed by numerical integration. Crank and Henry (1949a, 1949b) used the iteration scheme for several $D(\theta)$ functions, and found convergence to be quite good for diffusion coefficients not depending too strongly on $\theta$. If $D_{\min }$ and $D_{\max }$ are fairly close together, then the lower and upper bounds $\mathrm{v}_{1}(\phi)=\operatorname{erfc}\left(\phi / 2\left(\mathrm{D}_{\min }\right)^{\frac{1}{2}}\right)$ and $\mathrm{s}_{1}(\phi)=\operatorname{erfc}\left(\phi / 2\left(\mathrm{D}_{\max }\right)^{\frac{1}{2}}\right)$ are quite close together, and we may use a solution of the problem with constant "average" D as a starting point. When the bounds are further apart, speed of convergence is greatly increased by careful choice of starting function. It may be seen from (41) that in fact we are trying to guess the function $\mathrm{D}(\mathrm{s}(\phi))$, so in general when $\mathrm{dD} / \mathrm{ds}$ is large, accurate choice of $\mathrm{s}_{0}$ becomes important. Klute $(1952 \mathrm{a}, 1952 \mathrm{~b})$ used the method for the absorption of water into soil, where $\mathrm{D}_{\min }$ and $\mathrm{D}_{\max }$ may vary by several orders of magnitude and found convergence to be very slow. 


\section{CHAPTER 4}

\section{AN ANALYTICAL SOLUTION BY PERTURBATION METHOD}

\subsection{APPLICATION OF THE KIRCHHOFF TRANSFORMATION}

In this chapter we use perturbation methods to derive a general analytical solution to the simple one-dimensional diffusion equation in a semi-infinite region with constant initial concentration and constantconcentration boundary conditions when the diffusion coefficient has a simple power-law or exponential variation with concentration. Because we apply the Kirchhoff transformation before expanding in a series of powers of a small parameter $\alpha$, the zero-order solution with $\alpha=0$ when transformed transformed back to a function $\theta_{0}(\phi)$ is not the ordinary constant-D solution $\theta(\phi)=\theta_{0}+\left(\theta_{1}-\theta_{0}\right) \operatorname{erfc}\left(\phi / 2 \bar{D}^{-\frac{1}{2}}\right)$, but is an improvement on it.

The forms of $D-\theta$ relation we shall examine here are the power-law and exponential relationships, namely

and

$$
D(\theta)=a \theta^{k}
$$

$$
D(\theta)=b \exp (c \theta) \text {, }
$$

where $a, k, b$ and $c$ are positive constants. We assume throughout that $\theta>0$ are in the range of consideration.

Kidder (1957) developed a perturbation method, carried out to include terms of the second order, for calculating the solution to the desorption problem $\left(\theta_{0}>\theta_{1}\right)$, in the particular case $D(\theta)=a \theta$. We shall show how the perturbation method can be generalized to provide solutions to both the absorption and desorption problems, for the more general D - $\theta$ relations given above.

The $D-\theta$ relations can be written in the forms 


$$
D(\theta)=D_{0}\left(\theta / \theta_{0}\right)^{k}
$$

and

$$
D(\theta)=D_{0}^{\left(\theta_{1}-\theta / \theta_{1}-\theta_{0}\right)} D_{1}^{\left(\theta-\theta_{0} / \theta_{1}-\theta_{0}\right)}
$$

respectively, where $D_{0}=D\left(\theta_{0}\right), D_{1}=D\left(\theta_{1}\right)$.

For the absorption case, $\theta_{1}>\theta_{0}>0$, apply the Kirchhoff transformation in the form

$$
u(\theta)=\frac{\int_{\theta}^{\theta_{1}} D(a) d a}{\int_{\theta_{0}^{2}}^{\theta_{2}} D(a) d a} .
$$

For the power-law diffusivity, this gives

Put

$$
u(\theta)=\frac{\theta_{1}^{k+1}-\theta^{k+1}}{\theta_{1}^{k+1}-\theta_{0}^{k+1}} .
$$

$$
\alpha=\frac{\theta_{1}^{k+1}-\theta_{0}^{k+1}}{\theta_{1}^{k+1}} .
$$

The relation between $\mathrm{D}$ and $\mathrm{u}$ becomes

$$
D(u)=D_{1}(1-\alpha u)^{k /(k+1)} .
$$

With exponential diffusivity, this becomes

Put

$$
u(\theta)=\frac{D_{i}-D(\theta)}{D_{i}-D_{0}} .
$$

$$
\alpha=\frac{D_{1}-D_{0}}{D_{1}} \text {. }
$$

In this case the relation between $\mathrm{D}$ and $\mathrm{u}$ becomes

$$
D(u)=D(1-\alpha u) .
$$

We notice that the D-u relation for exponential diffusivity may be obtained from that for power-law diffusivity by symbolically "letting $k$ approach infinity" in the power-law relationship. In this sense, then, the exponential diffusivity function is the "limiting case" of the power-law function.

If we write the one-dimensional diffusion equation (10) in terms of $u$, we obtain 


$$
D(u) \frac{d^{2} u}{d \phi^{2}}+\frac{\phi}{2} \frac{d u}{d \phi}=0 \text {, }
$$

with boundary conditions $\mathrm{u}=0$ when $\phi=0, \mathrm{u} \rightarrow 1$ as $\phi \rightarrow \infty$. Equivalently,

$$
\frac{d^{2} u}{d \phi^{2}}+\frac{\phi}{2 D_{1}}(1-\alpha u)^{-p} \frac{d u}{d \phi}=0
$$

where $p=k /(k+1)$ for power-law diffusivity, and $p=1$ for exponential diffusivity.

Putting

$$
\begin{gathered}
\xi=\frac{\phi}{2 D_{1}^{\frac{1}{2}}} \\
\frac{d^{2} u}{d \xi^{2}}+2 \xi(1-\alpha u)^{-p} \frac{d u}{d \xi}=0
\end{gathered}
$$

with the conditions $u=0$ when $\xi=0$, and $u \rightarrow 1$ as $\xi \rightarrow \infty$.

For desorption, $\theta_{0}>\theta_{1}>0$, use the Kirchhoff transformation in the form

$$
v(\theta)=\frac{\int_{\theta}^{\theta_{0}} D(a) d a}{\int_{\theta_{1}}^{\theta_{0}} D(a) d a} .
$$

For the power-law diffusivity, this gives

Putting

$$
v(\theta)=\frac{\theta_{0}^{k+1}-\theta^{k+1}}{\theta_{0}^{k+1}-\theta_{1}^{k+1}} .
$$

we get

$$
\alpha=\frac{\theta_{0}^{k+1}-\theta_{1}^{k+1}}{\theta_{0}^{k+1}},
$$

$$
D(v)=D_{0}(1-\alpha v)^{k /(k+1)} .
$$

For the exponential diffusivity, this gives

$$
v(\theta)=\frac{D_{0}-D(\theta)}{D_{0}-D_{1}} .
$$

Putting

$$
\alpha=\frac{D_{0}-D_{1}}{D_{0}}
$$

we get

$$
D(v)=D_{0}(1-\alpha v)
$$

For desorption we put

$$
\xi=\frac{\phi}{2 D_{0}^{\frac{1}{2}}}
$$


and proceeding as in the absorption case, we get

$$
\frac{d^{2} v}{d \xi^{2}}+2 \xi(1-\alpha v)^{-p} \frac{d v}{d \xi}=0 \text {, }
$$

where $p=k /(k+1)$ or 1 as before.

The boundary conditions corresponding to desorption are $\mathrm{v}=0$ when $\xi=0$, and $v \rightarrow 1$ as $\xi \rightarrow \infty$. Note that in equations $(48)-(50),(52)-(54)$, it is always the larger of $D_{0}$ and $D_{1}$ which occurs explicitly. This is necessary to ensure that $0<\alpha<1$.

\subsection{THE PERTURBATION EXPANSION}

On physical grounds we require that $0 \leq \mathrm{u}(\phi) \leq 1$, and when $0<\alpha<1$ equation (51) satisfies all the hypotheses of the Existence Theorem of Chapter 3. Therefore we know that a solution with certain upper and lower bounds exists. The Seyferth uniqueness theorem holds, and ensures uniqueness.

As $0<\alpha<1$, and $0 \leq \mathrm{u} \leq 1$, the term $(1-\alpha \mathrm{u})^{-\mathrm{p}}$ may be expanded as a Taylor series in $\alpha$,

$$
(1-\alpha u)^{-p}=1+p u \alpha+\frac{p(p+1)}{2} u^{2} \alpha^{2}+\ldots .
$$

Using the small parameter method of Poincaré (1892), we may express $\mathrm{u}$ as a Taylor series in $\alpha$,

$$
u=u_{0}+u_{1} \alpha+u_{2} \alpha^{2}+\cdots .
$$

Substituting the series representations (55) and (56) into equation (51), we get

$$
\begin{aligned}
\frac{d^{2} u_{0}}{d \xi^{2}}+\alpha \frac{d^{2} u_{1}}{d \xi^{2}}+\alpha^{2} \frac{d^{2} u_{2}}{d \xi^{2}}+\ldots+2 \xi\left[\frac{d u_{0}}{d \xi}+\alpha \frac{d u_{1}}{d \xi}+\alpha^{2} \frac{d u_{2}}{d \xi}\right] . \\
{\left[1+p \alpha\left(u_{0}+\alpha u_{1}+\alpha^{2} u_{2}+\ldots\right)+\frac{p(p+1)}{2} \alpha^{2}\left(u_{0}^{2}+\ldots\right)\right]=0 . }
\end{aligned}
$$

Equating coefficients of the various powers of $\alpha$ to zero, we obtain equations for the $u_{j}, j=0,1,2 \ldots$, 


$$
\frac{d^{2} u_{0}}{d \xi^{2}}+2 \xi \frac{d u_{0}}{d \xi}=0
$$

with boundary conditions $u_{0}(0)=0, u_{0}(\infty)=1$

$$
\frac{d^{2} u_{1}}{d \xi^{2}}+2 \xi \frac{d u_{1}}{d \xi}=-p \xi \frac{d}{d \xi}\left(u_{0}^{2}\right)
$$

with boundary conditions $u_{1}(0)=0, u_{1}(\infty)=0$

$$
\frac{d^{2} u_{2}}{d \xi^{2}}+2 \xi \frac{d u_{2}}{d \xi}=-2 p \xi \frac{d}{d \xi}\left(u_{0} u_{i}\right)-\frac{p(p+1)}{3} \xi \frac{d}{d \xi}\left(u_{0}^{3}\right)
$$

with boundary conditions $u_{2}(0)=0, u_{2}(\infty)=0$. For absorption, we have

$$
u_{0}=\operatorname{erf}(\xi) \equiv \frac{2}{\pi^{\frac{1}{2}}} \int_{0}^{\xi} \exp \left(-a^{2}\right) d a .
$$

To solve equation (58) for $u_{1}$, we use the integrating factor $\Phi \equiv \exp \left(-\xi^{2}\right)$. The solution is

$$
u_{1}=A+B u_{0}-p \int \Phi \int \Phi^{-1}\left\{\xi \frac{d}{d \xi}\left(u_{0}^{2}\right)\right\},
$$

where $\mathrm{A}$ and $\mathrm{B}$ are constants chosen to satisfy the boundary conditions. After evaluation and simplification,

$$
u_{1}=-p\left(\frac{1}{2 \pi}\right)\left\{1-\pi^{\frac{1}{2}} \xi \exp \left(-\xi^{2}\right) u_{0}-\exp \left(-2 \xi^{2}\right)\right\} .
$$

The equation for $u_{2}$ now becomes

$$
\begin{gathered}
\frac{d^{2} u_{2}}{d \xi^{2}}+2 \xi \frac{d u_{2}}{d \xi}=p^{2}\left\{\frac{2}{\pi^{3 / 2}} \exp \left(-\xi^{2}\right)-\frac{1}{\pi^{\frac{1}{2}}}\left(\xi-2 \xi^{3}\right) \exp \left(-\xi^{2}\right) u_{0}^{2}\right. \\
\left.-\frac{2}{\pi^{3 / 2}} \xi \exp \left(-3 \xi^{2}\right)\right\}-p(p+1)\left\{\frac{2}{\pi^{\frac{1}{2}}} \exp \left(-\xi^{2}\right) u_{0}^{2}\right\} .
\end{gathered}
$$

The solution satisfying the boundary conditions is found to be

$$
\begin{aligned}
u_{2}=p^{2}\{ & -\frac{1}{2 \pi^{3 / 2}} \xi \exp \left(-\xi^{2}\right)-\frac{1}{8 \pi^{\frac{1}{2}}}\left(\xi+2 \xi^{3}\right) \exp \left(-\xi^{2}\right) u_{0}^{2} \\
& \left.-\frac{1}{2 \pi}\left(1+\xi^{2}\right) \exp \left(-2 \xi^{2}\right) u_{0}+\frac{3 \sqrt{3}}{8 \pi}\left(\operatorname{erf}(\xi \sqrt{3})-u_{0}\right)\right\} \\
& +p(p+1)\left\{\frac{1}{2 \pi^{\frac{1}{2}} \xi \exp \left(-\xi^{2}\right) u_{0}^{2}}+\frac{1}{\pi} \exp \left(-2 \xi^{2}\right) u_{0}\right. \\
& \left.-\frac{\sqrt{3}}{2 \pi}\left(\operatorname{erf}(\xi \sqrt{3})-u_{0}\right)\right\} .
\end{aligned}
$$


Although $u_{3}$ and higher terms may be calculated in the same manner, the calculations rapidly become very complex.

For desorption, we have

$$
\begin{gathered}
\frac{d^{2} v_{0}}{d \xi^{2}}+2 \xi \frac{d v_{0}}{d \xi}=0, \\
v_{0}(0)=1, \quad v_{0}(\infty)=0 .
\end{gathered}
$$

Therefore

$$
\begin{gathered}
\mathrm{v}_{0}=\operatorname{erfc}(\xi) \equiv 1-\frac{2}{\pi^{\frac{1}{2}}} \int_{0}^{\xi} \exp \left(-\mathrm{a}^{2}\right) \mathrm{da} \\
\frac{\mathrm{d}^{2} \mathrm{v}_{1}}{\mathrm{~d} \xi^{2}}+2 \xi \frac{\mathrm{d} \mathrm{v}_{1}}{\mathrm{~d} \xi}=-\mathrm{p} \xi \frac{\mathrm{d}}{\mathrm{d} \xi}\left(\mathrm{v}_{0}^{2}\right), \\
\mathrm{v}_{1}(0)=0, \quad \mathrm{v}_{1}(\infty)=0 .
\end{gathered}
$$

The solution satisfying the boundary conditions is

$$
\mathrm{v}_{1}=\mathrm{p}\left\{-\frac{1}{\pi} \mathrm{v}_{0}\left(1+\pi^{\frac{1}{2}} \xi \exp \left(-\xi^{2}\right)\right)+\frac{1}{\pi} \exp \left(-2 \xi^{2}\right)\right\} .
$$

The equation for $v_{2}$ becomes

$$
\begin{aligned}
\frac{d^{2} v_{2}}{d \xi^{2}}+ & 2 \xi \frac{d v_{2}}{d \xi}=p^{2}\left\{\frac{2}{\pi} \xi \frac{d}{d \xi}\left(v_{0}^{2}\right)+\frac{2}{\pi^{\frac{1}{2}}}\left(\xi-2 \xi^{3}\right) \exp \left(-\xi^{2}\right) v_{0}^{2}\right. \\
& \left.+\frac{4}{\pi^{3 / 2}} \xi \exp \left(-3 \xi^{2}\right)\right\}+p(p+1)\left\{\frac{2}{\pi^{\frac{1}{2}}} \xi \exp \left(-\xi^{2}\right) v_{0}^{2}\right\},
\end{aligned}
$$

with

$$
\mathrm{v}_{2}(0)=0, \quad \mathrm{v}_{2}(\infty)=0 .
$$

The solution satisfying the boundary condition is

$$
\begin{aligned}
\mathrm{v}_{2} & =\mathrm{p}^{2}\left\{\left(\frac{2}{\pi^{2}}\right)\left[\mathrm{v}_{0}\left(1+\pi^{\frac{1}{2}} \xi \exp \left(-\xi^{2}\right)\right)-\exp \left(-2 \xi^{2}\right)\right]+\frac{4}{\pi} \mathrm{v}_{0}\right. \\
+ & \frac{1}{2 \pi^{3 / 2}} \xi \exp \left(-3 \xi^{2}\right)-\frac{1}{\pi}\left(4+\xi^{2}\right) \exp \left(-2 \xi^{2}\right) \mathrm{v}_{0}+\frac{1}{4 \pi^{\frac{1}{2}}}\left(\xi+2 \xi^{3}\right) \exp \left(-\xi^{2}\right) \mathrm{v}_{0}^{2} \\
- & \left.\frac{7 \sqrt{3}}{4 \pi}(\operatorname{erf}(\xi \sqrt{3})-\operatorname{erf}(\xi))\right\}+\mathrm{p}(\mathrm{p}+1)\left\{-\frac{2}{\pi} \mathrm{v}_{0}+\frac{2}{\pi} \exp \left(-2 \xi^{2}\right) \mathrm{v}_{0}\right. \\
& \left.-\frac{1}{2 \pi^{\frac{1}{2}}} \xi \exp \left(-\xi^{2}\right) \mathrm{v}_{0}^{2}+\frac{5 \sqrt{3}}{6 \pi}(\operatorname{erf}(\xi \sqrt{3})-\operatorname{erf}(\xi))\right\} .
\end{aligned}
$$

In the special case $p=\frac{1}{2}$, the above results for desorption reduce to those of Kidder.

We now write our results in terms of $\theta$. For absorption, 
and

$$
\left(\theta_{1}>\theta_{0}\right), \quad \xi=\frac{\phi}{2 D_{1}^{\frac{1}{2}}},
$$

$$
\theta=\theta_{1}\left(1-\alpha u_{0}-\alpha^{2} u_{1}-\alpha^{3} u_{2}-\ldots\right)^{1 /(k+1)}
$$

with

$$
\alpha=\frac{\theta_{1}^{k+1}-\theta_{0}^{k+1}}{\theta_{1}^{k+1}},
$$

when the diffusion coefficient is of power-law form. When the diffusion coefficient is of exponential form,

where

$$
\theta=\theta_{1}-\frac{\left(\theta_{1}-\theta_{0}\right)}{\log \left(D_{0} / D_{1}\right)} \log \left(1-\alpha u_{0}-\alpha^{2} u_{1}-\alpha^{3} u_{2}-\ldots\right),
$$

$$
\alpha=\frac{D_{1}-D_{0}}{D_{1}} .
$$

For desorption

and

$$
\left(\theta_{0}>\theta_{1}\right), \quad \xi=\frac{\phi}{2 D_{0}^{\frac{1}{2}}},
$$

$$
\theta=\theta_{0}\left(1-\alpha v_{0}-\alpha^{2} v_{1}-\alpha^{3} v_{2}-\ldots\right)^{1 /(k+1)},
$$

where

$$
\alpha=\frac{\theta_{0}^{\mathrm{k}+1}-\theta_{1}^{\mathrm{k}+1}}{\theta_{0}^{\mathrm{k}+1}}
$$

for power-law diffusion coefficient.

For exponential diffusion coefficient,

where

$$
\theta=\theta_{0}-\frac{\left(\theta_{0}-\theta_{1}\right)}{\log \left(D_{1} / D_{0}\right)} \log \left(1-\alpha v_{0}-\alpha^{2} v_{1}-\alpha^{3} v_{2}-\ldots\right),
$$

$$
\alpha=\frac{D_{0}-D_{1}}{D_{0}} .
$$

In fact, $u_{0}, u_{1}, u_{2}$, and $v_{0}, v_{1}, v_{2}$ may be expressed in the form

$$
\begin{array}{lll}
\mathrm{u}_{0}=\mathrm{u}_{0}(\xi), & \mathrm{u}_{1}=\mathrm{pf}_{1}(\xi), & \mathrm{u}_{2}=\mathrm{p}^{2} \mathrm{f}_{2}(\xi)+\mathrm{p}(\mathrm{p}+1) f_{3}(\xi) \\
\mathrm{v}_{0}=\mathrm{v}_{0}(\xi), & \mathrm{v}_{1}=\mathrm{pg}_{1}(\xi), & \mathrm{v}_{2}=\mathrm{p}^{2} \mathrm{~g}_{2}(\xi)+\mathrm{p}(\mathrm{p}+1) g_{3}(\xi)
\end{array}
$$

and $f_{1}, f_{2}, f_{3}, g_{1}, g_{2}, g_{3}$ can be tabulated as functions of $\xi$ for a range of values of $\xi$. The appropriate values of $\alpha$ and $p$ may then be used to calculate numerical solutions to the whole range of problems described herein. Kidder (1957) has calculated numerical values of $v_{0}, v_{1}$ and $v_{2}$ 
for the case $p=\frac{1}{2}$. His Table 1 is reproduced below.

Table 1 (Kidder, 1957)

Numerical Values of Coefficients in

Equations (62) and (63), for $p=\frac{1}{2}$

\begin{tabular}{|c|ccc|}
\hline $\begin{array}{c}\text { Argument } \\
\xi\end{array}$ & $\mathrm{v}_{0}(\xi)$ & $\mathrm{v}_{1}(\xi)$ & $\mathrm{v}_{2}(\xi)$ \\
\hline 0.0 & 1.00000 & 0.00000 & 0.00000 \\
0.1 & 0.88754 & -0.01004 & -0.00343 \\
0.2 & 0.77730 & -0.01893 & -0.00611 \\
0.3 & 0.67137 & -0.02584 & -0.00766 \\
0.4 & 0.57161 & -0.03037 & -0.00809 \\
0.5 & 0.47950 & -0.03245 & -0.00763 \\
0.6 & 0.39614 & -0.03236 & -0.00661 \\
0.7 & 0.32220 & -0.03052 & -0.00534 \\
0.8 & 0.25790 & -0.02748 & -0.00410 \\
0.9 & 0.20309 & -0.02376 & -0.00299 \\
1.0 & 0.15730 & -0.01982 & -0.00210 \\
1.2 & 0.08969 & -0.01253 & -0.00094 \\
1.5 & 0.03389 & -0.00514 & -0.00025 \\
2.0 & 0.00468 & -0.00074 & -0.00003 \\
\hline
\end{tabular}

Convergence can be seen to be quite good even for $\alpha$ near 1 , which corresponds to $D_{0} \gg D_{1}$. The quantities in Table 1 are respectively the coefficients of $\alpha, \alpha^{2}$, and $\alpha^{3}$, so for $\alpha<\frac{1}{2}$ say, which corresponds to $\mathrm{D}_{0}=2 \mathrm{D}_{1}$, the contribution of the term $\mathrm{v}_{2}(\xi)$ to $\theta$ is negligible.

The series solutions (60)-(64) add to the armoury of D- $\theta$ functions which can be fitted to experimental $D-\theta$ functions, in order to obtain approximate $\theta-\phi$ profiles. Their area of usefulness overlaps partly with that of the iteration scheme of Crank and Henry, but they have the advantage that in problems for which the power-law or exponential diffusion coefficient is appropriate, the tedious numerical calculation may be done once and for all, and then the $\theta-\phi$ profiles may be easily 
calculated for a wide range of values of $\theta_{0}, \theta_{1}$ and $k$ merely by varying $\alpha$ and $\mathrm{p}$. 


\section{CHAPTER 5}

\section{SOME NEW EXACT SOLUTIONS FOR A NONLINEAR DIFFUSION EQUATION WITH CONSTANT-FLUX BOUNDARY CONDITION}

\subsection{THE METHOD OF STORM}

Storm (1951) considered the problem of conduction of heat in a metal, when both the thermal conductivity $k$ and the heat capacity $C$ are functions of temperature. He showed that when $k$ and $C$ satisfy a certain relation, the nonlinear equation for conduction of heat in a semiinfinite medium with the flux specified at the surface can be transformed to a linear equation.

Storm stated that the corresponding problem for the diffusion equation can be solved by his methods when the diffusion coefficient is an exponential function of concentration. This is not so, as the coefficients $C(T)=1, k(T)=a \exp (b \theta)$ in the diffusion equation considered as a heat equation do not satisfy Storm's criterion.

However the method can be used to provide an exact solution to the problem of one-dimensional diffusion into a semi-infinite region with the flux specified on the boundary, when the diffusion coefficient has the form

$$
D(\theta)=a(1-b \theta)^{-2},
$$

where $\mathrm{a}$ and $\mathrm{b}$ are constants, with $\mathrm{a}>0$ and $\theta \neq \mathrm{b}^{-1}$.

Fujita (1952) found that the above $D-\theta$ relation satisfactorily described experimental data such as that of Crank and Park (1949), to which Park (1950) fitted an exponential $D(\theta)$ relationship. 
In expression (64) the diffusivity increases very rapidly for $\theta$ near $\mathrm{b}^{-1}$, and becomes infinite at $\theta=b^{-1}$. Therefore this $D-\theta$ relation is appropriate to experimental $D-\theta$ relations in which $D(\theta)$ increases very rapidly near some finite value of $\theta$.

The equation to be solved is

$$
\frac{\partial}{\partial x}\left(a(1-b \theta)^{-2} \frac{\partial \theta}{\partial x}\right)=\frac{\partial \theta}{\partial t}
$$

with initial and boundary conditions

$$
\begin{aligned}
& \theta=\theta_{0} \text { when } t=0, \quad\left(\theta_{0}<b^{-1}\right) \text {, }
\end{aligned}
$$

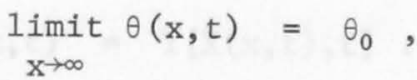

$$
\begin{aligned}
& \left(a(1-b \theta)^{-2} \frac{\partial \theta}{\partial x}\right)_{x=0}=-f,
\end{aligned}
$$

where $a, b, \theta_{0}$ and $f$ are constants, and $a, b$ are positive.

Apply the transformation of Kirchhoff (1894)

This gives

$$
s(\theta)=\int_{\theta_{0}}^{\theta} a(1-b u)^{-2} d u .
$$

$$
s(\theta)=\frac{a}{b}\left[(1-b \theta)^{-1}-\left(1-b \theta_{0}\right)^{-1}\right] .
$$

The equation to be solved becomes

$$
a\left\{\frac{b s}{a}+\left(1-b \theta_{0}\right)^{-1}\right\}^{2} \frac{\partial^{2} s}{\partial x^{2}}=\frac{\partial s}{\partial t}
$$

with boundary conditions

$$
\begin{gathered}
s=0 \text { when } t=0, \\
\underset{x \rightarrow 0}{\operatorname{limit}} s(x, t)=0, \\
\left.\frac{\partial s}{\partial x}\right|_{x=0}=-f .
\end{gathered}
$$

Apply the linear transformation

$$
\mathrm{u}=\frac{\mathrm{bs}\left(1-\mathrm{b} \theta_{0}\right)}{\mathrm{a}}+1 .
$$

The equation becomes 


$$
a\left(1-b \theta_{0}\right)^{-2} u^{2} \frac{\partial^{2} u}{\partial x^{2}}=\frac{\partial u}{\partial t}
$$

with initial and boundary conditions

$$
\begin{gathered}
u=1 \text { when } t=0, \\
u \rightarrow 1 \quad \text { as } \quad x \rightarrow \infty, \\
\left.\frac{\partial u}{\partial x}\right|_{x=0}=\frac{-\left(1-b \theta_{0}\right) b f}{a} .
\end{gathered}
$$

Make the change of variables

and write

$$
x(x, t)=\int_{0}^{x} \frac{\left(1-b \theta_{0}\right)}{a^{\frac{1}{2}} u\left(x^{\prime}, t\right)} d x^{\prime},
$$

$$
\mathrm{u}(\mathrm{x}, \mathrm{t})=\mathrm{Y}[\mathrm{X}(\mathrm{x}, \mathrm{t}), \mathrm{t}] .
$$

Now

$$
\frac{\partial u}{\partial x}=\left(\frac{\partial Y}{\partial X}\right)\left(\frac{\partial X}{\partial x}\right)=\frac{\left(1-b \theta_{0}\right)}{a^{\frac{1}{2}} u}\left(\frac{\partial Y}{\partial X}\right)
$$

and

$$
\frac{\partial^{2} u}{\partial x^{2}}=\frac{\left(1-b \theta_{0}\right)^{2}}{a u^{2}}\left(\frac{\partial^{2} Y}{\partial X^{2}}\right)-\frac{\left(1-b \theta_{0}\right)}{a^{\frac{1}{2}} u^{2}}\left(\frac{\partial u}{\partial x}\right)\left(\frac{\partial Y}{\partial X}\right) \text {. }
$$

Therefore

$$
a\left(1-b \theta_{0}\right)^{-2} u^{2} \frac{\partial^{2} u}{\partial x^{2}}=\frac{\partial^{2} Y}{\partial X^{2}}-\frac{a^{\frac{1}{2}}}{\left(1-b \theta_{0}\right)}\left(\frac{\partial u}{\partial x}\right)\left(\frac{\partial Y}{\partial X}\right) \text {. }
$$

Now

$$
\begin{aligned}
\frac{\partial u}{\partial t} & =\frac{\partial Y}{\partial t}+\left(\frac{\partial Y}{\partial X}\right)\left[\int_{0}^{x}\left\{\frac{-\left(1-b \theta_{0}\right)}{a^{\frac{1}{2}} u^{2}} \frac{\partial u}{\partial t}\right\} d x\right] \\
& =\frac{\partial Y}{\partial t}-\left(\frac{\partial Y}{\partial X}\right)\left[\frac{a^{\frac{1}{2}}}{\left(1-b \theta_{0}\right)} \int_{0}^{x} \frac{\partial^{2} u}{\partial x^{2}} d x\right] \\
& =\frac{\partial Y}{\partial t}-\frac{a^{\frac{1}{2}}}{\left(1-b \theta_{0}\right)}\left(\frac{\partial Y}{\partial X}\right)\left[\frac{\partial u}{\partial x}-\left.\frac{\partial u}{\partial x}\right|_{x=0}\right] .
\end{aligned}
$$

Using conditions (72),

$$
\frac{\partial u}{\partial t}=\frac{\partial Y}{\partial t}-\frac{a^{\frac{1}{2}}}{\left(1-b \theta_{0}\right)}\left(\frac{\partial u}{\partial x}\right)\left(\frac{\partial Y}{\partial X}\right)-\frac{b f}{a^{\frac{1}{2}}}\left(\frac{\partial Y}{\partial X}\right) .
$$

Combining (75) and (76), and using (71)

$$
\frac{\partial Y}{\partial t}=\frac{\partial^{2} Y}{\partial X^{2}}+\frac{b f}{a^{\frac{1}{2}}} \frac{\partial Y}{\partial X} \text {. }
$$

The boundary conditions (72) become 


$$
\begin{gathered}
Y=1 \text { when } t=0, \\
Y \rightarrow 1 \text { as } X \rightarrow \infty, \\
\quad\left(\frac{1}{Y} \frac{\partial Y}{\partial X}\right)_{X=0}=-\frac{b f}{a^{\frac{1}{2}}} .
\end{gathered}
$$

Note that the equation and the "radiation" boundary condition are linear in $\mathrm{Y}$.

Introduce the dimensionless variables

$$
\eta=-\frac{b f X}{a^{\frac{1}{2}}}, \quad \tau=-\frac{b f t^{\frac{1}{2}}}{a^{\frac{1}{2}}}, \quad x=-\frac{b f\left(1-b \theta_{0}\right) x}{a} \text {. }
$$

The solution of (77) subject to (78) is (Storm, 1951)

$$
\begin{aligned}
Y= & 1+\frac{1}{2} \exp (n)\left[\tau^{2}+1 \cdot+n\right] \operatorname{erfc}(n / 2 \tau+\tau / 2) \\
& -\frac{1}{2} \operatorname{erfc}(n / 2 \tau-\tau / 2)-\left(\tau / \pi^{\frac{1}{2}}\right) \exp \left[-(n / 2 \tau-\tau / 2)^{2}\right] .
\end{aligned}
$$

Inverting equation (73) gives

or

$$
\frac{d x}{d X}=\frac{a^{\frac{1}{2}} Y(X, t)}{\left(1-b \theta_{0}\right)}
$$

Integrating (79),

$$
\mathrm{x}=\frac{\mathrm{a}^{\frac{1}{2}}}{\left(1-\mathrm{b} \theta_{0}\right)} \int_{0}^{\mathrm{X}} \mathrm{Y} \mathrm{dX} \text {. }
$$

$x=\frac{1}{2} \exp (n)\left[n+\tau^{2}\right] \operatorname{erfc}(n / 2 \tau+\tau / 2)+\left[n-\tau^{2}\right]\left[1-\frac{1}{2} \operatorname{erfc}(n / 2 \tau-\tau / 2)\right]$

Expressions (79) and (80) give $Y$ and $X$ in terms of the parameter $n$ and the variable $\tau$, so we have an implicit relation between $u, x$ and $t$. From equation (70),

$$
s=\frac{a(u-1)}{b\left(1-b \theta_{0}\right)} .
$$

This gives from (79),

$$
\begin{aligned}
\mathbf{s}= & \frac{a}{2 b\left(1-b \theta_{0}\right)}\left\{\exp (n)\left[\tau^{2}+1+n\right] \operatorname{erfc}(n / 2 \tau+\tau / 2)\right. \\
& \left.-\operatorname{erfc}(n / 2 \tau-\tau / 2)-\left(2 \tau / \pi^{\frac{1}{2}}\right) \exp \left[-(n / 2 \tau-\tau / 2)^{2}\right]\right\} .
\end{aligned}
$$

Inverting (67),

$$
\theta(X, t)=b^{-1}\left\{1-\frac{\left(1-b \theta_{0}\right)}{\left(\frac{b\left(1-b \theta_{0}\right) s(X, t)}{a}+1\right)}\right\} \text {. }
$$


This is the solution to equation (65) satisfying conditions (66). Note that $\theta(x, t)<b^{-1}$ for all finite $t$. In physical terms we have that near $b^{-1}$ the diffusion coefficient becomes infinite sufficiently rapidly to enable any finite flux density to be transmitted with $\theta<b^{-1}$.

This situation corresponds roughly, though not exactly, to a "saturation concentration" in a real medium.

When the diffusivity is nearly constant, that is when $b \ll 1$, and $x$ and $t$ are small, we have $x \approx a^{\frac{1}{2}} x$ in $(80)$. Also $s(\theta) \approx a\left(\theta-\theta_{0}\right)$, and expression (81) reduces to the correct solution for the case $D(\theta)=a$, with the same boundary and initial conditions.

\subsection{APPLICATION OF THE METHOD TO MORE GENERAL PROBLEMS}

In the previous theory we notice that when $f=0$, that is when

$$
\left(a(1-b \theta)^{-2} \frac{\partial \theta}{\partial x}\right)_{x=0}=0 \text {, }
$$

equation (77) reduces to the ordinary heat equation

$$
\frac{\partial Y}{\partial t}=\frac{\partial^{2} Y}{\partial X^{2}} \text {. }
$$

This means that the method of Storm may be used to solve problems with more general initial and boundary conditions, provided that the condition for zero flux at $\mathrm{x}=0$ is maintained, either by an "insulating barrier" at $\mathrm{x}=0$ in a semi-infinite region, or by a symmetry of initial conditions about $\mathrm{x}=0$ in an infinite region.

The problem described by equation (65) and the initial conditions $\theta(x, 0)=g(x), \theta_{0} \leq g(x)<b^{-1}$ for some $\theta_{0}$, and boundary conditions

$$
\left(a(1-b \theta)^{-2} \frac{\partial \theta}{\partial x}\right)=0 \text { at } x=0
$$

may be transformed to a problem

$$
\frac{\partial Y}{\partial t}=\frac{\partial^{2} Y}{\partial X^{2}}
$$


with conditions

$$
\begin{aligned}
& Y(X, 0)=W(X) \\
& \left.\frac{\partial Y}{\partial X}\right|_{X=0}=0 .
\end{aligned}
$$

This problem may be solved by considering it to be a problem in an infinite region with initial condition

$$
\begin{array}{ll}
Y(X, 0)=W(X) & \text { for } X \geq 0, \\
Y(X, 0)=W(-X) & \text { for } X<0 .
\end{array}
$$

For $W$ sufficiently well-behaved, the solution is (Carslaw and Jaeger, 1959)

Therefore

$$
\begin{aligned}
Y(X, t)= & \frac{1}{2 \pi^{\frac{1}{2}} t^{\frac{1}{2}}} \int_{0}^{\infty} W(\xi) \exp \left[-(X-\xi)^{2} / 4 t\right] d \xi \\
& +\frac{1}{2 \pi^{\frac{1}{2}} t^{\frac{1}{2}}} \int_{-\infty}^{0} W(-\xi) \exp \left[-(X-\xi)^{2} / 4 t\right] d \xi .
\end{aligned}
$$

$$
Y(X, t)=\frac{1}{2 \pi^{\frac{1}{2}} t^{\frac{1}{2}}} \int_{0}^{\infty} W(\xi)\left\{\exp \left[-(X-\xi)^{2} / 4 t\right]+\exp \left[-(X+\xi)^{2} / 4 t\right]\right\} d \xi .
$$

As before, we can now transform back to $\theta(x, t)$.

As an example, consider the problem in the infinite region with initial conditions

$$
\theta(x, 0)= \begin{cases}\theta_{0} & x<-c \\ \theta_{1} & -c \leq x \leq c \\ \theta_{0} & x>c\end{cases}
$$

where $c$ is a positive constant and $\theta_{0}<\theta_{1}<b^{-1}$. This problem is equivalent to a "redistribution problem" in a semi-infinite region with no flow at the surface.

The initial conditions must be expressed in terms of $\mathrm{Y}$ and $\mathrm{X}$, via $\mathrm{s}$ and $u$. When $t=0$,

$$
u(x, 0)=\left\{\begin{array}{cl}
\frac{1-b \theta_{0}}{1-b \theta_{1}} & \text { when }|x| \leq c \\
1 & \text { when }|x|>c .
\end{array}\right.
$$


The relation (73) gives

Therefore

$$
X(x, 0)=\frac{\left(1-b \theta_{0}\right)}{a^{\frac{1}{2}}} \int_{0}^{x} \frac{d x^{\prime}}{u\left(x^{\prime}, 0\right)} .
$$

$$
X(x, 0)=\left\{\begin{array}{ll}
\frac{\left(1-b \theta_{1}\right) x}{a^{\frac{1}{2}}} & \text { when }|x| \leq c \\
\frac{\left(1-b \theta_{0}\right) x-b c\left(\theta_{1}-\theta_{0}\right)}{a^{\frac{1}{2}}} & \text { when }|x|>c .
\end{array} .\right.
$$

In particular

$$
X(c, 0)=\frac{\left(1-b \theta_{1}\right) c}{a^{\frac{1}{2}}} .
$$

Therefore the initial conditions become

Write

$$
\mathrm{Y}[\mathrm{X}(\mathrm{x}, 0), 0]=\left\{\begin{array}{ccc}
\frac{1-\mathrm{b} \theta_{0}}{1-\mathrm{b} \theta_{1}} & \text { when } & |\mathrm{X}| \leq \frac{\left(1-\mathrm{b} \theta_{1}\right) c}{\mathrm{a}^{\frac{1}{2}}} \\
1 & \text { when } & |\mathrm{X}|>\frac{\left(1-\mathrm{b} \theta_{1}\right) c}{\mathrm{a}^{\frac{1}{2}}}
\end{array} .\right.
$$

$$
B=\frac{\left(1-b \theta_{1}\right) c}{a^{\frac{1}{2}}} \text {. }
$$

The solution to (83) subject to conditions (85) is (Carslaw and Jaeger, 1959)

$$
Y(X, t)=1+\frac{b\left(\theta_{1}-\theta_{0}\right)}{\left(1-b \theta_{1}\right)}\left\{\frac{1}{2} \operatorname{erf}\left(\frac{B-X}{2 t^{\frac{1}{2}}}\right)+\frac{1}{2} \operatorname{erf}\left(\frac{B+X}{2 t^{\frac{1}{2}}}\right)\right\} .
$$

The relation

gives

$$
x=\frac{a^{\frac{1}{2}}}{\left(1-b \theta_{0}\right)} \int_{0}^{X} Y d X
$$

$$
\begin{aligned}
& x(x, t)=\frac{a^{\frac{1}{2}} X}{\left(1-b \theta_{0}\right)}+\frac{a^{\frac{1}{2}} b\left(\theta_{1}-\theta_{0}\right)}{\left(1-b \theta_{0}\right)\left(1-b \theta_{1}\right)}\left\{\frac{1}{2}(B+X) \operatorname{erf}\left(\frac{B+X}{2 t^{\frac{1}{2}}}\right)\right. \\
& \left.-\frac{1}{2}(B-X) \operatorname{erf}\left(\frac{B-X}{2 t^{\frac{1}{2}}}\right)+\left(\frac{t}{\pi}\right)^{\frac{1}{2}}\left[\exp \left(\frac{-(B+X)^{2}}{4 t}\right)-\exp \left(\frac{-(B-X)^{2}}{4 t}\right)\right]\right\} .
\end{aligned}
$$

Proceeding as before, 
and

$$
s(X, t)=\frac{a\left(\theta_{1}-\theta_{0}\right)}{\left(1-b \theta_{0}\right)\left(1-b \theta_{1}\right)}\left\{\frac{1}{2} \operatorname{erf}\left(\frac{B-X}{2 t^{\frac{1}{2}}}\right)+\frac{1}{2} \operatorname{erf}\left(\frac{B+X}{2 t^{\frac{1}{2}}}\right)\right\}
$$

$$
\theta(X, t)=b^{-1}\left\{1-\frac{\left(1-b \theta_{0}\right)}{\left(\frac{b\left(1-b \theta_{0}\right) s(X, t)}{a}+1\right)}\right\} .
$$

Equations (87) and (88) enable the calculation of $\theta$ as a function of $x$ and $t$ to give the final solution to the problem.

\section{$5.2 \frac{\text { AN EXACT SOLUTION CORRESPONDING }}{\text { TO AN INSTANTANEOUS PLANE SOURCE }}$}

A "source solution" to the problem

is

$$
\frac{\partial Y}{\partial t}=\frac{\partial^{2} Y}{\partial X^{2}}
$$

$$
\operatorname{limit}_{t \rightarrow \infty}[Y(X, t)]=1,\left.\quad \frac{\partial Y}{\partial X}\right|_{X=0}=0 \text {, }
$$

$$
Y(X, t)=1+\frac{A b}{2(\pi a t)^{\frac{1}{2}}} \exp \left(-X^{2} / 4 t\right)
$$

where $\mathrm{A}$ is a constant. Therefore

$$
x(X, t)=\frac{a^{\frac{1}{2}} X}{\left(1-b \theta_{0}\right)}+\frac{A b}{2\left(1-b \theta_{0}\right)} \operatorname{erf}\left(\frac{X}{2 t^{\frac{1}{2}}}\right) .
$$

Relation (82) gives

$$
\theta(x, t)=b^{-1}\left[1-\frac{\left(1-b \theta_{0}\right)}{\left(1+\frac{A b}{2(\pi a t)^{\frac{1}{2}}} \exp \left(-\frac{x^{2}}{4 t}\right)\right)}\right] .
$$

Equations (89) and (90) enable calculation of $\theta$ as a function of $x$ and $t$.

In three dimensions the problem corresponds to the instantaneous release at time zero of A units of diffusing substance per unit area over the surface of the plane $x=0$, with constant initial concentration $\theta_{0}$ throughout the rest of the infinite region. The solution also applies to the problem in the semi-infinite region with a no-flux condition at $x=0$, with A/2 units of diffusing substance released at the boundary at time zero. 


\subsection{THE SOLUTION TO THE MOST GENERAL PROBLEM WITH CONSTANT-FLUX BOUNDARY CONDITION, AND EXTENSIONS TO VARIABLE FLUX}

The problem with general initial concentration, and constant-flux boundary condition at $\mathrm{x}=0$, reduces to the linear equation

$$
\frac{\partial Y}{\partial t}=\frac{\partial^{2} Y}{\partial X^{2}}+\beta \frac{\partial Y}{\partial X}
$$

with boundary conditions

$$
\left.\left(\frac{\partial Y}{\partial X}+\beta Y\right)\right|_{X=0}=0
$$

and initial condition

$$
\mathrm{Y}(\mathrm{X}, 0)=\mathrm{Y}_{0}(\mathrm{X})
$$

where $\beta$ is a constant, the value $\beta=0$ corresponding to a no-flux boundary condition.

Put

$$
Y(X, t)=\exp (-\beta X / 2) V(X, t) \exp \left(-\beta^{2} t / 4\right)
$$

Then

subject to

$$
\frac{\partial^{2} V}{\partial X^{2}}=\frac{\partial V}{\partial t}
$$

$$
\left.\left(\frac{\partial V}{\partial X}+\frac{\beta}{2} V\right)\right|_{X=0}=0
$$

and

$$
\mathrm{V}(\mathrm{X}, 0)=\exp (\beta X / 2) \mathrm{Y}_{0}(\mathrm{X})
$$

The solution of (95) for a unit instantaneous plane source at $X^{\prime}$ at time zero, satisfying boundary condition (96) is (Carslaw and Jaeger, 1959)

$$
\begin{aligned}
G_{\beta}\left(X, X^{\prime}, t\right)= & \frac{1}{2(\pi t)^{\frac{1}{2}}}\left\{\exp \left[\frac{-\left(X-X^{\prime}\right)^{2}}{4 t}\right]+\exp \left[\frac{-\left(X+X^{\prime}\right)^{2}}{4 t}\right]\right\} \\
& +\frac{\beta}{2} \exp \left[t \frac{\beta^{2}}{4}-\frac{\beta}{2}\left(X+X^{\prime}\right)\right] \operatorname{erfc}\left(\frac{X+X^{\prime}}{2 t^{\frac{1}{2}}}-\frac{\beta}{2} t^{\frac{1}{2}}\right] .
\end{aligned}
$$

When $Y(X, 0)$ is suitably well behaved, the solution to (95) with conditions (96) and (97) is

$$
V(X, t)=\int_{0}^{\infty} G_{\beta}\left(X, X^{\prime}, t\right) \exp \left(\beta X^{\prime} / 2\right) Y_{0}\left(X^{\prime}\right) d X^{\prime} .
$$

Therefore the solution of (91) subject to (92) and (93) is 


$$
\exp \left(\beta^{2} t / 4\right) Y(X, t)=\int_{0}^{\infty} G_{\beta}\left(X, X^{\prime}, t\right) \exp \left[-\frac{\beta}{2}\left(X-X^{\prime}\right)\right] Y_{0}\left(X^{\prime}\right) d X^{\prime}
$$

where $G_{\beta}$ is given by (98).

This solution may be used with the relation

$$
x(X, t)=\frac{a^{\frac{1}{2}}}{\left(1-b \theta_{0}\right)} \int_{0}^{X} Y\left(X^{\prime}, t\right) d X^{\prime}
$$

to give expressions for $\theta$ and $\mathrm{x}$ in terms of the parameter $\mathrm{X}$ and the variable $t$.

As noted by Storm, the transformation of the original nonlinear equation with nonlinear boundary condition to a linear equation with linear boundary condition applies equally well when the flux on the boundary is not constant, but is a function of time. In this case the coefficient $\beta$ in (91) and (92) is a function of the variable $t$. The resultant equation is thus linear, but with a non-constant coefficient, and so in general is not easy to solve.

In the special case that the boundary flux is proportional to $t^{-\frac{1}{2}}$, and the initial concentration is constant, we have a similarity solution of (91) subject to (92) and (93). Putting $\Phi=X t^{-\frac{1}{2}}$, we may assume that $Y$ is a function only of $\Phi$. Equation (91) reduces to an ordinary differential equation, whose solution can be used to give expressions for $\theta$ and $\mathrm{x}$ in terms of the parameter $\Phi$ and the variable $t$. By similarity the problem with diffusion coefficient $D(\theta)=a(1-b \theta)^{-2}$, constant initial concentration $\theta_{0}$, and boundary flux proportional to $t^{-\frac{1}{2}}$ is equivalent to the problem considered by Fujita (1952b) with the same diffusion coefficient and initial concentration, and constant boundary concentration $\theta_{1}$. Therefore we have shown how the method of Storm (1951) may be used to derive the solution of Fujita (1952b) to the latter problem. 
Some indications of the behaviour of solutions of (91) with boundary flux as a periodic function of the time may be obtained by considering the problem in which $\beta$ in (91) and (92) is a "square-wave" function of time with period $2 \mathrm{~T}$, namely

$$
\beta(t)=\left\{\begin{array}{rrrr}
F & \text { when } & 2 n T \leq t<(2 n+1) T, & n=0,1 \ldots, \\
-F & \text { when } & (2 n+1) T \leq t<(2 n+2) T, & n=0,1 \ldots .
\end{array}\right.
$$

Equation (100) gives an expression for $\mathrm{Y}(\mathrm{X}, \mathrm{T})$ in terms of the initial value $Y(X, 0)$, and for $Y(X,(n+1) T)$ in terms of $Y(X, n T)$. Equivalently, to find the "quasi-steady state" reached at large time, using (100) twice we may write down an integral equation involving $\mathrm{Y}(\mathrm{X}, \mathrm{nT})=\mathrm{Y}(\mathrm{X},(\mathrm{n}+2) \mathrm{T})$, and solve this integral equation using the method of successive approximations.

The basis of Storm's method is the transformation of the equation (71) to a linear equation. The condition given by Storm for the heat equation to be transformed to a linear equation can be shown to be equivalent to the condition that the heat equation

$$
C(T) \frac{\partial T}{\partial t}=\frac{\partial}{\partial x}\left(k(T) \frac{\partial T}{\partial x}\right)
$$

be transformed by the Kirchhoff transformation

$$
S(T)=\int_{T_{0}}^{T} k\left(T^{\prime}\right) d T^{\prime}
$$

to the form of equations (68) or (71). This criterion is easier to apply in practice than that of Storm.

Therefore the solutions given in this chapter for various problems for the particular diffusion equation considered, all apply to the heat equation, when the coefficients satisfy the above condition. 


\subsection{THE WORK OF PARLANGE}

Recently, Parlange (1971a, 1971b, 1971c) described a new iterative method for solving equations (1) and (3) with one space variable, and constant concentration boundary conditions. The method does not assume that $D$ is nearly constant, and so is suitable for widely-varying $D(\theta)$ functions. We investigate the application of this method to the simplest problem, that is described by the equation,

$$
\frac{\partial \theta}{\partial t}=\frac{\partial}{\partial x}\left(D(\theta) \frac{\partial \theta}{\partial x}\right)
$$

with boundary conditions,

$$
\begin{aligned}
& \theta=\theta_{0} \text { when } t=0, x>0 \\
& \theta=\theta_{1} \text { when } t \geq 0, x=0 .
\end{aligned}
$$

Parlange uses equation (102) in the inverted form due to Philip, namely

$$
\frac{\partial x}{\partial t}+\frac{\partial}{\partial \theta}\left(D(\theta) / \frac{\partial x}{\partial \theta}\right)=0
$$

where $t$ and $\theta$ are now the independent variables, and $x$ is the dependent variable.

The surface flux is an as yet unknown function of time. Integrating equation (103) we get

Putting

$$
\int_{\theta}^{\theta_{1}}\left(\frac{\partial x}{\partial t}\right) d \theta+D\left(\theta_{1}\right) /\left.\frac{\partial x}{\partial \theta}\right|_{\theta=\theta_{1}}-D(\theta) / \frac{\partial x}{\partial \theta}=0 .
$$

$$
1 / g(t)=D\left(\theta_{1}\right) /\left.\frac{\partial x}{\partial \theta}\right|_{\theta=\theta_{1}}
$$

and using the condition 
we have from (104),

$$
D\left(\theta_{0}\right) /\left.\frac{\partial x}{\partial \theta}\right|_{\theta=\theta_{0}}=0,
$$

$$
\int_{\theta_{0}}^{\theta_{1}}\left(\frac{\partial x}{\partial t}\right) d \theta+1 / g(t)=0 \text {. }
$$

Parlange makes the approximation that there is constant flux density throughout the region of influence, which gives

$$
D(\theta) / \frac{\partial x}{\partial \theta}=1 / g(t) .
$$

This means that with the condition that $\mathrm{x}=0$ when $\theta=\theta_{1}$, we have

$$
x=-g(t) \int_{\theta}^{\theta_{1}} D(a) d a
$$

Substituting (107) in (105) gives the differential equation for $g(t)$,

with condition

$$
\frac{1}{g}=\frac{d g}{d t} \int_{\theta_{0}}^{\theta_{1}} \theta D(\theta) d \theta
$$

$$
g(0)=0 \text {. }
$$

Hence,

$$
\mathrm{g}^{2}=2 t /\left(\int_{\theta_{0}}^{\theta_{1}} \theta \mathrm{D}(\theta) \mathrm{d} \theta\right)
$$

and so the first approximation of Parlange is

$$
x_{1}(\theta, t)=\frac{\sqrt{2 t} \int_{\theta}^{\theta_{1}} D(a) d a}{\left(\int_{\theta_{0}}^{\theta_{1}} a D(a) d a\right)^{\frac{1}{2}}} .
$$

The first approximation is, in fact, identical to the approximate solution of Macey (1959) to this problem.

Parlange then derives a second approximation to the solution by substituting the expression for $x(\theta, t)$ given by (108) into equation (103) to obtain the relation

$$
\frac{\int_{\theta}^{\theta_{1}} D(a) d a}{\left(2 t \int_{\theta_{0}}^{\theta_{1}} a D(a) d a\right)^{\frac{1}{2}}}+\frac{\partial}{\partial \theta}\left(D(\theta) / \frac{\partial x}{\partial \theta}\right)=0 .
$$

The conditions 


$$
D\left(\theta_{0}\right) /\left.\frac{\partial x}{\partial \theta}\right|_{\theta=\theta_{0}}=0,
$$

and $x\left(\theta_{1}, t\right)=0$, are used to derive the second approximation,

$$
x_{2}(\theta, t)=\sqrt{2 t}\left(\int_{\theta_{0}}^{\theta_{1}} \mathrm{aD}(\mathrm{a}) \mathrm{da}\right) \int_{\theta}^{\theta_{1}} \frac{\mathrm{D}(\gamma) \mathrm{d} \gamma}{\int_{\theta_{0}}^{\gamma} \int_{\beta}^{\theta_{1}} D(a) d a d \beta} .
$$

Parlange states that because the first approximation satisfies equation (105) which is a type of overall conservation condition, then we expect the second approximation to be very close to the exact solution. In fact, the second approximation does not satisfy the "integral continuity" constraint defined by (105), so in this sense it is not as good an approximation as the first. Successive approximations are derived by the same method that is used to obtain the second from the first.

In his Table 2, Parlange (1971, p.136) compares values of the exact solution for constant $D$, with values of the second approximation (110) for the same case, and concludes that the agreement of the second approximation with the exact solution is fairly good. However, if both sets of figures are compared with the first approximation (108) for constant D, it can be seen that the second approximation has a better "shape" than the first, but that the second approximation is demonstrably worse than the first in terms of percentage error.

\subsection{THE PROBLEM AS AN INTEGRAL EQUATION}

It is much easier to see the mathematical meaning of Parlange's method of solving equation (102) if we use the Boltzmann similarity substitution, and then write the problem as an integral equation.

Putting $\phi=x t^{-\frac{1}{2}}$, we have

$$
\frac{d}{d \phi}\left(D(\theta) \frac{d \theta}{d \phi}\right)+\frac{\phi}{2} \frac{d \theta}{d \phi}=0
$$


where $\phi$ is the independent variable, and $\theta$ the dependent variable. We know from Chapter 2 that when $D(\theta)$ is sufficiently well-behaved, $\theta$ is a continuous strictly decreasing function of $\phi$ on $[0, \infty)$. Therefore we can make $\theta$ the independent and $\phi$ the dependent variable in (111). The function $\phi(\theta)$ is defined for $\theta \in\left(\theta_{0}, \theta_{1}\right]$, with $\phi \rightarrow \infty$ as $\theta \rightarrow \theta_{0}$.

Putting $G(\theta)=D(\theta) \frac{\mathrm{d} \theta}{\mathrm{d} \phi}$, we have

$$
\frac{\mathrm{d} \phi}{\mathrm{d} \theta} \cdot \frac{\mathrm{d}}{\mathrm{d} \phi}(G(\theta))+\frac{\phi}{2}=0
$$

or

$$
\frac{\mathrm{d} G}{\mathrm{~d} \theta}+\frac{\phi}{2}=0
$$

Differentiating,

or

$$
\frac{d^{2} G}{d \theta^{2}}+\frac{1}{2} \frac{d \phi}{d \theta}=0
$$

$$
\frac{d^{2} G}{d \theta^{2}}+\frac{1}{2} \frac{D(\theta)}{G(\theta)}=0 .
$$

This is a nonlinear second-order ordinary differential equation in $G(\theta)$. From equation (112), we see that the requirement that $\phi=0$ when $\theta=\theta_{1}$ becomes the condition

$$
\frac{\mathrm{d} G}{\mathrm{~d} \theta}=0 \text { when } \theta=\theta_{1} \text {. }
$$

The conditions $\phi \rightarrow \infty$ as $\theta \rightarrow \theta_{0}, D(\theta) \frac{d \theta}{d \phi} \rightarrow 0$ as $\phi \rightarrow \infty$. mean that

$$
G\left(\theta_{0}\right)=0 \text {. }
$$

The function $\phi(\theta)$ is then given by

$$
\phi(\theta)=\int_{\theta}^{\theta_{1}} \frac{D(\theta)}{G(\theta)} d \theta .
$$

Equation (113) and conditions (114) and (115) define a nonlinear two-point boundary value problem, which we may examine using the methods of Bailey, Shampine and Waltman (1968). We know from the Existence Theorem for equation (111), that condition (112) implies that $\mathrm{dG} / \mathrm{d} \theta \rightarrow \infty$ as $\theta \rightarrow \theta_{0}$. 
Using the appropriate Green's function for the boundary conditions, we may transform equation (113) with conditions (114) and (115) into an integral equation. The Green's function is (Bailey, Shampine and Waltman, 1968, p.24)

$$
H(\theta, a)=\left\{\begin{array}{ll}
a-\theta_{0} & \theta_{0} \leq a \leq \theta \leq \theta_{1} \\
\theta-\theta_{0} & \theta_{0} \leq \theta \leq a \leq \theta_{1} .
\end{array} .\right.
$$

The integral equation is

Explicitly

$$
G(\theta)=\int_{\theta_{0}}^{\theta_{1}} \frac{H(\theta, a) D(a) d a}{2 G(a)} .
$$

In particular

$$
G(\theta)=\int_{\theta_{0}}^{\theta} \frac{\left(a-\theta_{0}\right) D(a) d a}{2 G(a)}+\int_{\theta}^{\theta_{1}} \frac{\left(\theta-\theta_{0}\right) D(a) d a}{2 G(a)} .
$$

$$
G\left(\theta_{1}\right)=\int_{\theta_{0}}^{\theta_{1}} \frac{\left(a-\theta_{0}\right) D(a) d a}{2 G(a)} .
$$

Picard (1893) showed that we may solve (117) by the method of successive approximations, and that the iteration scheme converges for any suitable starting guess, when the function $D(\theta) / 2 G$ satisfies a Lipschitz condition in G, and the Lipschitz constant is small enough. Since $G(\theta) \rightarrow 0$ as $\theta \rightarrow \theta_{0}$, we do not have a constant $K$ such that for all $G_{1}, G_{2} \geq 0$,

$$
\left|\frac{D(\theta)}{2 G_{1}}-\frac{D(\theta)}{2 G_{2}}\right| \leq K\left|G_{1}-G_{2}\right|
$$

and so in general we do not expect convergence.

If we write the iteration scheme of Parlange in terms of $G(\theta)$ rather that $\phi(\theta)$, it is equivalent to Picard's method of successive approximations applied to the above integral equation, with $G_{1}(\theta)$ chosen such that $G_{1}(\theta)=A$, where $A$ is a constant such that $G_{1}\left(\theta_{1}\right)=G_{2}\left(\theta_{1}\right)=A$. That the Parlange scheme does not converge in the conventional sense may be shown by a simple argument.

If the Picard iteration scheme converges for a certain starting function $G_{1}(\theta)$, then it must also converge for any other starting 
function sufficiently close to $G_{1}(\theta)$. Suppose that $\alpha$ is a constant close to, but not equal to, unity.

Put

$$
E_{1}(\theta)=\alpha G_{1}(\theta) .
$$

Then

$$
E_{2}(\theta)=\alpha^{-1} G_{2}(\theta),
$$

and

$$
E_{3}(\theta)=\alpha G_{3}(\theta) .
$$

If $G_{n}(\theta) \rightarrow G(\theta)$ as $n \rightarrow \infty$, then

and

$$
E_{n}(\theta) \rightarrow \alpha G(\theta) \quad \text { for } n \text { odd }
$$

$$
E_{n}(\theta) \rightarrow \alpha^{-1} G(\theta) \text { for } n \text { even. }
$$

Putting

$$
\phi_{n}(\theta)=\int_{\theta}^{\theta_{1}} \frac{D(a) d a}{E_{n}(a)}
$$

we see that the successive functions $\phi_{n}(\theta)$ oscillate about the exact solution $\phi(\theta)$, and do not converge when $\alpha \neq 1$.

The calculations of Knight and Philip (1973) for two different $D(\theta)$ functions indicate that the successive approximations of Parlange for these problems do in fact oscillate about the true solution in this manner, the "shapes" of successive approximations changing until a steady oscillation occurs.

\subsection{IMPROVEMENT OF THE PARLANGE METHOD}

The characteristic oscillation of Parlange's scheme may be eliminated by requiring that successive estimates $G_{n}$ and $G_{n+1}$ of $G$ satisfy the constraint of "integral continuity", $G_{n}\left(\theta_{1}\right)=G_{n+1}\left(\theta_{1}\right)$. We use the flux-concentration relation of Philip (1973).

Put

$$
F(\theta)=\frac{G(\theta)}{G\left(\theta_{1}\right)} \text {. }
$$


Then,

$$
F\left(\theta_{0}\right)=0, \quad F\left(\theta_{1}\right)=1 \text { and } F^{\prime}\left(\theta_{1}\right)=0 \text {. }
$$

Put

$$
Q^{*}=G\left(\theta_{1}\right)=\int_{\theta_{0}}^{\theta_{1}} \frac{\left(a-\theta_{0}\right) D(a) d a}{2 G(a)} .
$$

From equation (118), we get

$$
\begin{aligned}
F(\theta) & =\frac{\int_{\theta_{0}}^{\theta} \frac{\left(a-\theta_{0}\right) D(a) d a}{2 G(a)}+\int_{\theta}^{\theta_{1}} \frac{\left(\theta-\theta_{0}\right) D(a) d a}{2 G(a)}}{\int_{\theta_{0}}^{\theta_{1}} \frac{\left(a-\theta_{0}\right) D(a) d a}{2 G(a)}} \\
& =\frac{Q^{*}-\int_{\theta}^{\theta_{1}} \frac{\left(a-\theta_{0}\right) D(a) d a}{2 G(a)}+\int_{\theta}^{\theta_{1}} \frac{\left(\theta-\theta_{0}\right) D(a) d a}{2 G(a)}}{Q^{*}} \\
& =\frac{Q^{*}-\int_{\theta}^{\theta_{1}} \frac{(a-\theta) D(a) d a}{2 G(a)}}{Q^{*}} \\
& =1-\frac{\int_{\theta}^{\theta_{1}} \frac{(a-\theta) D(a) d a}{2 G(a)}}{\int_{\theta_{0}}^{\theta_{1}} \frac{\left(a-\theta_{0}\right) D(a) d a}{2 G(a)}} .
\end{aligned}
$$

We now put

$$
\begin{aligned}
& F_{n+1}(\theta)=1-\frac{\int_{\theta}^{\theta_{1}} \frac{(a-\theta) D(a) d a}{F_{n}(a)}}{\int_{\theta_{0}}^{\theta_{1}} \frac{\left(a-\theta_{0}\right) D(a) d a}{F_{n}(a)}} \\
& G_{n+1}(\theta)=\int_{\theta_{0}}^{\theta_{1}} \frac{H(\theta, a) D(a) d a}{2 G_{n}(a)}
\end{aligned}
$$

and

$$
Q_{n}^{*}=G_{n}\left(\theta_{1}\right)=Q_{n}^{*} F_{n}\left(\theta_{1}\right)
$$

Then the "integral continuity" requirement that $G_{n}\left(\theta_{1}\right)=G_{n+1}\left(\theta_{1}\right)$ is equivalent to $Q_{n}^{*}=Q_{n+1}^{*}$. Since

$$
\begin{aligned}
Q_{n+1}^{*}=G_{n+1}\left(\theta_{1}\right) & =\int_{\theta_{0}}^{\theta_{1}} \frac{\left(a-\theta_{0}\right) D(a) d a}{2 G_{n}(a)} \\
& =\int_{\theta_{0}}^{\theta_{1}} \frac{\left(a-\theta_{0}\right) D(a) d a}{2 Q_{n}^{*} F_{n}(a)}
\end{aligned}
$$

Therefore,

$$
2 Q_{n+1}^{*} Q_{n}^{*}=\int_{\theta_{0}}^{\theta_{1}} \frac{\left(a-\theta_{0}\right) D(a) d a}{F_{n}(a)}
$$


and so integral continuity requires that $G_{n}$ be such that

Therefore

$$
G_{n}\left(\theta_{1}\right)=G_{n+1}\left(\theta_{1}\right)=\left(\frac{1}{2} \int_{\theta_{0}}^{\theta_{1}} \frac{\left(a-\theta_{0}\right) D(a) d a}{F_{n}(a)}\right)^{\frac{1}{2}} .
$$

$$
\phi_{n}(\theta)=\frac{\int_{\theta}^{\theta_{1}} \frac{D(a) d a}{F_{n}(a)}}{\left(\frac{1}{2} \int_{\theta_{0}}^{\theta_{1}} \frac{\left(a-\theta_{0}\right) D d a}{F_{n}(a)}\right)^{\frac{1}{2}}} .
$$

If we put $F_{1}(\theta)=1$ in equation $(121), \phi_{1}(\theta)$ is identical to Parlange's first approximation, but higher approximations differ from those of Parlange. The improved iteration scheme given by (120) eliminates the inherent oscillation of Parlange's iteration scheme.

A general proof that the sequences of functions given by (120) and (121) converge to provide a solution to (111) has not yet been obtained. A proof due to Philip that the sequence of functions given by (120) converges when $D(\theta)=\theta^{m}-\theta^{2 m} /(1+m), m>0$, is given in Philip and Knight (1973). Numerical results in particular cases indicate that convergence is quite rapid (Philip and Knight, 1973).

Apart from the obvious improvement produced by eliminating the inherent oscillations of Parlange's scheme, the Green's Function-Integral Equation formulation has various advantages.

The systematic iterative nature of Parlange's scheme is more clearly demonstrated. It is evident that Parlange's choice of $F_{1}=1$ is not necessary. Indeed, the work of Philip (1973) indicates that for D a strongly varying function of $\theta$, the choice $F_{1}(\theta)=1$ is "best" only for the case of desorption $\left(D\left(\theta_{0}\right)>D\left(\theta_{1}\right)\right)$. For absorption $\left(D\left(\theta_{0}\right)<D\left(\theta_{1}\right)\right)$, a much better $F_{1}$ for this one-dimensional case is $F_{1}(\theta)=\left(\theta-\theta_{0}\right) /\left(\theta_{1}-\theta_{0}\right)$.

In practice, the quadratures involved in equations (120) and (121) must be performed by some scheme for numerical integration. The Green's function formulation requires approximately half the number of 
quadratures needed by Parlange's formulation (110). We do not have to calculate the value of $\phi_{n}$, either explicitly or implicitly, until successive values of $F_{n}$ are seen to agree to within the required degree of accuracy.

Parlange applied his technique to more complex problems in (1971a, 1971b) and subsequent works. In the complex situations where we do not have similarity, the behaviour of Parlange's iteration schemes is much more difficult to investigate.

In a more general problem, the flux-concentration relation varies with time, and it is necessary to formulate the problem as a partial integro-differential equation, rather than as a simple integral equation.

In the limit of small time, the various problems considered by Parlange (1971b, 1971c) reduce to the simple case considered in (1971a).

Therefore, the convergence of the uncorrected Parlange schemes for the more complex problems is certainly no better than the convergence in the simpler case, and is probably worse.

When successive iterates have been constrained to satisfy integral continuity, the more complex relationship between each iterate and the next means that it is essential to choose a first approximation which makes the second approximation very close to the exact solution. Some further work along these lines is reported in Philip and Knight (1973).

In order to solve the "infiltration" equation (3) in the onedimensional form

$$
\frac{\partial \theta}{\partial t}=\frac{\partial}{\partial z}\left(D(\theta) \frac{\partial \theta}{\partial z}\right)-\frac{d K}{d \theta} \cdot \frac{\partial \theta}{\partial z}
$$

where $\mathrm{z}$ is the vertical ordinate, we use the inverted form due to Philip, name $1 \mathrm{y}$

$$
\frac{\partial z}{\partial t}+\frac{\partial}{\partial \theta}\left(D(\theta) / \frac{\partial z}{\partial \theta}\right)-\frac{d K}{d \theta}=0
$$


Integrating,

$$
\int_{\theta_{0}}^{\theta}\left(\frac{\partial z}{\partial t}\right) d \theta+D(\theta) / \frac{\partial z}{\partial \theta}-K(\theta)+K\left(\theta_{0}\right)=0 .
$$

In particular, we have the overall conservation condition

Put

$$
\int_{\theta_{0}}^{\theta_{1}}\left(\frac{\partial z}{\partial t}\right) d \theta+D\left(\theta_{1}\right) /\left.\frac{\partial z}{\partial \theta}\right|_{\theta=\theta_{1}}-K\left(\theta_{1}\right)+K\left(\theta_{0}\right)=0 .
$$

and

$$
v(\theta, t)=K(\theta)-D(\theta) / \frac{\partial z}{\partial \theta}=\int_{\theta_{0}}^{\theta}\left(\frac{\partial z}{\partial t}\right) d \theta-K\left(\theta_{0}\right)
$$

$$
q(t)=v\left(\theta_{1}, t\right)-K\left(\theta_{0}\right)=\int_{\theta_{0}}^{\theta_{1}}\left(\frac{\partial z}{\partial t}\right) d \theta-K\left(\theta_{0}\right) .
$$

The flux-concentration relation of Philip (1973) for this problem is

$$
F(\theta, t)=\frac{v(\theta, t)-K\left(\theta_{0}\right)}{v\left(\theta_{1}, t\right)-K\left(\theta_{0}\right)}=\frac{\int_{\theta_{0}}^{\theta}\left(\frac{\partial z}{\partial t}\right) d \theta}{\int_{\theta_{0}}^{\theta_{1}}\left(\frac{\partial z}{\partial t}\right) d \theta} .
$$

Here the flux-concentration relation varies with time. Its behaviour is investigated in Philip (1973). As $t \rightarrow 0, F(\theta, t) \rightarrow F$ abs $(\theta)$, where $F_{a b s}(\theta)$ satisfies equation (119) and is the flux-concentration relation associated with the simpler "absorption" problem. As $t \rightarrow \infty$, $F(\theta, t) \rightarrow\left(\theta-\theta_{0}\right) /\left(\theta_{1}-\theta_{0}\right)$. For all positive $t, F(\theta, t)$ lies between these limiting values.

Put $k(\theta)=K(\theta)-K\left(\theta_{0}\right)$.

We have

$$
z(\theta, t)=\int_{\theta}^{\theta_{1}} \frac{D(a) d a}{v(a, t)-K(a)}=\int_{\theta}^{\theta_{1}} \frac{D(a) d a}{q(t) F(a, t)-k(a)} .
$$

Substituting (127) in (126) gives an integro-differential equation for $F(\theta, t)$, namely

$$
F(\theta, t)=\frac{\int_{\theta_{0}}^{\theta} \int_{\theta}^{\theta_{1}} D(a) \frac{\partial}{\partial t}\left(\frac{1}{q(t) F(a, t)-k(a)}\right) d a d \theta}{\int_{\theta_{0}}^{\theta}\left(\theta-\theta_{0}\right) D(\theta) \frac{\partial}{\partial t}\left(\frac{1}{q(t) F(\theta, t)-k(\theta)}\right) d \theta} .
$$

We have from (125) the overall conservation requirement 


$$
q(t)=\int_{\theta_{0}}^{\theta_{1}}\left(\theta-\theta_{0}\right) D(\theta) \frac{\partial}{\partial t}\left(\frac{1}{q(t) F(\theta, t)-k(\theta)}\right) d \theta .
$$

As in the simpler "absorption" case already considered, we wish to construct an iteration scheme for $F(\theta, t)$ which does not require the calculation of $z(\theta, t)$ or $q(t)$ for each iterate.

Therefore we make the flux-concentration relation a function of $q$, and write (128) in terms of $q$ rather than $t$. The resulting expression for $F(\theta, q)$ is

Then

$$
F(\theta, q)=1-\frac{\int_{\theta}^{\theta_{1}} \frac{(a-\theta) D(a) \partial(q F(a, q)) / \partial q}{(q F(a, q)-k(a))^{2}} d a}{\int_{\theta_{0}}^{\theta_{1}} \frac{\left(\theta-\theta_{0}\right) D(\theta) \partial(q F(\theta, q)) / \partial q}{(q F(\theta, q)-k(\theta))^{2}} d \theta} .
$$

and

$$
z(\theta, q)=\int_{\theta}^{\theta_{1}} \frac{D(a) d a}{q F(a, q)-k(a)}
$$

$$
\frac{d t}{d q}=-\int_{\theta_{0}}^{\theta_{1}} \frac{\left(\theta-\theta_{0}\right) D(\theta) \partial(q F(\theta, q)) / \partial q}{q(q F(\theta, q)-k(\theta))^{2}} d \theta \text {. }
$$

Integrating with the condition $t=0$ when $q=\infty$,

$$
t(q)=\int_{\theta_{0}}^{\theta_{1}}\left(\theta-\theta_{0}\right) D(\theta) \int_{q^{\prime}}^{\infty} \frac{\left(\partial\left(q^{\prime} F\left(\theta, q^{\prime}\right)\right) / \partial q^{\prime}\right) d q^{\prime}}{q^{\prime}\left(q^{\prime} F\left(\theta, q^{\prime}\right)-k(\theta)\right)^{2}} d \theta .
$$

We construct an iterative scheme from (130) in the usual manner to give $F_{n+1}(\theta, q)$ in terms of $F_{n}(\theta, q)$. We then use (131) and (133) to calculate $z_{n+1}(\theta, q)$ and $t_{n+1}(q)$ when required. Parlange's first approximation for this problem can be obtained by putting $F_{1}(\theta, q)=1$ in (131) and (133). This gives

$$
\begin{aligned}
& z_{1}(\theta, q)=\int_{\theta}^{\theta_{1}} \frac{D(a) d a}{q-k(a)} \\
& t_{1}(q)=\int_{\theta_{0}}^{\theta_{1}} \frac{\left(\theta-\theta_{0}\right) D(\theta)}{(k(\theta))^{2}}\left(\frac{k(\theta)}{q-k(\theta)}-\ln \left(\frac{q}{q-k(\theta)}\right)\right) d \theta .
\end{aligned}
$$

Parlange's higher approximations are obtained by a method similar to that by which he obtains higher approximations in the absorption case already considered, and so suffer from the serious oscillations already 
mentioned, and certainly do not converge. In addition, he never changes his assumed $t(q)$ relation from that given by equation (135), which is of course only approximate. The choice $F_{1}(\theta, q)=1$ can certainly be improved upon, since we have seen that the upper and lower limits of $F(\theta, q)$ are respectively $\mathrm{F}_{\mathrm{abs}}$ and $\left(\theta-\theta_{0}\right) /\left(\theta_{1}-\theta_{0}\right)$.

The iteration scheme given here by (130), (131) and (133) does not have the above defects of Parlange's proposed scheme for solution of this problem. Because the expression (133) is in general not easy to evaluate, and because the whole question of convergence in the general case is difficult to investigate, we seek a very good choice of $F_{1}$ which avoids the need to iterate if possible. We can check the accuracy of $F_{1}$ by calculating $\mathrm{F}_{2}$ and comparing with $\mathrm{F}_{1}$.

We know that when $t$ ranges between 0 and $\infty$, $q$ ranges between $\infty$ and $k\left(\theta_{1}\right)=K\left(\theta_{1}\right)-K\left(\theta_{0}\right)$, and $F(\theta, q)$ ranges between $F_{a b s}(\theta)$ and $\left(\theta-\theta_{0}\right) /\left(\theta_{1}-\theta_{0}\right)$.

Therefore we can construct an "interpolated" $F_{1}(\theta, q)$ which simplifies (133) by putting

$$
\mathrm{F}_{1}(\theta, \mathrm{q})=\mathrm{F}_{\mathrm{abs}}(\theta)+\left(\left(\theta-\theta_{0}\right) /\left(\theta_{1}-\theta_{0}\right)-\mathrm{F}_{\mathrm{abs}}(\theta)\right) \mathrm{k}\left(\theta_{1}\right) / \mathrm{q} .
$$

This gives the correct form of $F$ at $q=\infty$ and at $q=k\left(\theta_{1}\right)$. Then

$$
\mathrm{qF}_{1}(\theta, \mathrm{q})-\mathrm{k}(\theta)=\mathrm{qF}_{\mathrm{abs}}(\theta)-\mathrm{k}^{\prime}(\theta)
$$

where

$$
k^{\prime}(\theta)=k(\theta)+k\left(\theta_{1}\right)\left(F_{a b s}(\theta)-\left(\theta-\theta_{0}\right) /\left(\theta_{1}-\theta_{0}\right)\right) .
$$

Substituting (137) in equations (131), (133), and (130) gives

$$
\begin{aligned}
z_{1}(\theta, q)= & \int_{\theta}^{\theta_{1}} \frac{D(a) d a}{q_{a b s}(a)-k^{\prime}(a)} \\
t_{1}(q)= & \int_{\theta_{0}}^{\theta_{1}} \frac{\left(\theta-\theta_{0}\right) D(\theta) F_{a b s}(\theta)}{\left(k^{\prime}(\theta)\right)^{2}}\left(\frac{k^{\prime}(\theta)}{q^{2}{ }_{a b s}(\theta)-k^{\prime}(\theta)}\right. \\
& \left.-\ln \left(\frac{\mathrm{qF}_{a b s}(\theta)}{\mathrm{qF}_{\mathrm{abs}}(\theta)-\mathrm{k}^{\prime}(\theta)}\right)\right] \mathrm{d} \theta .
\end{aligned}
$$




$$
F_{2}(\theta, q)=1-\frac{\int_{\theta}^{\theta_{1}} \frac{(a-\theta) D(a) F_{a b s}(a)}{\left(q F_{a b s}(a)-k^{\prime}(a)\right)^{2}} d a}{\int_{\theta_{0}}^{\theta_{1}} \frac{\left(\theta-\theta_{0}\right) D(\theta) F_{a b s}(\theta)}{\left(q F_{a b s}(\theta)-k^{\prime}(\theta)\right)^{2}} d \theta} .
$$

As a numerical example, expressions (138), (139) and (140) have been evaluated in Philip and Knight (1973) with the values of $D(\theta)$ and $K(\theta)$ for Yolo light clay used by Philip (1957) in his series solution to this problem. Numerical quadratures were performed on the CSIRO CDC 3600 computer, using the trapezoidal rule with 100 steps in each of $1 \geq\left(\theta-\theta_{0}\right) /\left(\theta_{1}-\theta_{0}\right) \geq 0.9$ and $0.1 \geq\left(\theta-\theta_{0}\right) /\left(\theta_{1}-\theta_{0}\right) \geq 0$, and 80 steps in $0.9 \geq\left(\theta-\theta_{0}\right) /\left(\theta_{1}-\theta_{0}\right) \geq 0.1$.

Table 2

Comparison of $F_{1}(\theta, q)$ and $F_{2}(\theta, q)$ for Yolo Light Clay

\begin{tabular}{|c|c|c|c|c|c|c|}
\hline \multirow[b]{2}{*}{$10^{6} \mathrm{q}$} & & \multicolumn{5}{|c|}{$\left(\theta-\theta_{0}\right) /\left(\theta_{1}-\theta_{0}\right)$} \\
\hline & & 0.9 & 0.7 & 0.5 & 0.3 & 0.1 \\
\hline$\infty$ & $\begin{array}{l}F_{1} \\
F_{2}\end{array}$ & $\begin{array}{l}0.964 \\
0.964\end{array}$ & $\begin{array}{l}0.818 \\
0.818\end{array}$ & $\begin{array}{l}0.638 \\
0.638\end{array}$ & $\begin{array}{l}0.418 \\
0.418\end{array}$ & $\begin{array}{l}0.153 \\
0.153\end{array}$ \\
\hline 257.4 & $\begin{array}{l}F_{1} \\
F_{2}\end{array}$ & $\begin{array}{l}0.960 \\
0.962\end{array}$ & $\begin{array}{l}0.813 \\
0.816\end{array}$ & $\begin{array}{l}0.631 \\
0.634\end{array}$ & $\begin{array}{l}0.412 \\
0.415\end{array}$ & $\begin{array}{l}0.151 \\
0.152\end{array}$ \\
\hline 74.23 & $\begin{array}{l}\mathrm{F}_{1} \\
\mathrm{~F}_{2}\end{array}$ & $\begin{array}{l}0.953 \\
0.960\end{array}$ & $\begin{array}{l}0.799 \\
0.809\end{array}$ & $\begin{array}{l}0.616 \\
0.626\end{array}$ & $\begin{array}{l}0.399 \\
0.407\end{array}$ & $\begin{array}{l}0.145 \\
0.149\end{array}$ \\
\hline 25.74 & $\begin{array}{l}\mathrm{F}_{1} \\
\mathrm{~F}_{2}\end{array}$ & $\begin{array}{l}0.933 \\
0.951\end{array}$ & $\begin{array}{l}0.762 \\
0.785\end{array}$ & $\begin{array}{l}0.572 \\
0.596\end{array}$ & $\begin{array}{l}0.362 \\
0.381\end{array}$ & $\begin{array}{l}0.128 \\
0.136\end{array}$ \\
\hline 18.018 & $\begin{array}{l}F_{1} \\
F_{2}\end{array}$ & $\begin{array}{l}0.920 \\
0.941\end{array}$ & $\begin{array}{l}0.738 \\
0.763\end{array}$ & $\begin{array}{l}0.544 \\
0.568\end{array}$ & $\begin{array}{l}0.337 \\
0.356\end{array}$ & $\begin{array}{l}0.117 \\
0.125\end{array}$ \\
\hline 13.445 & $\begin{array}{l}F_{1} \\
F_{2}\end{array}$ & $\begin{array}{l}0.906 \\
0.919\end{array}$ & $\begin{array}{l}0.710 \\
0.724\end{array}$ & $\begin{array}{l}0.512 \\
0.523\end{array}$ & $\begin{array}{l}0.310 \\
0.318\end{array}$ & $\begin{array}{l}0.105 \\
0.108\end{array}$ \\
\hline 12.288 & $\begin{array}{l}F_{1} \\
F_{2}\end{array}$ & $\begin{array}{l}0.900 \\
0.900\end{array}$ & $\begin{array}{l}0.700 \\
0.700\end{array}$ & $\begin{array}{l}0.500 \\
0.500\end{array}$ & $\begin{array}{l}0.300 \\
0.300\end{array}$ & $\begin{array}{l}0.100 \\
0.100\end{array}$ \\
\hline
\end{tabular}

Table 2 above compares $F_{1}(\theta, q)$ given by the formula (136) and the first iterate $F_{2}(\theta, q)$ given by equation (140), for various values of $q$. Agreement between $F_{1}$ and $F_{2}$ is of course exact for small and for large $q$, and can be seen to be very close for intermediate values of $q$. Therefore 
for practical purposes there is no need to iterate to improve the values of $z_{1}(\theta, q)$ and $t_{1}(q)$ given by expressions (138) and (139). In Philip and Knight (1973) the values of $z(\theta, q)$ and $t(q)$ obtained as above are compared with values obtained with other starting functions, and with values obtained by the method of Parlange. The values obtained from the interpolated $F_{1}(\theta, q)$ are very close to those obtained by the series solution of Philip (1957). 


\section{REFERENCES}

Abarbanel, S.S. (1960). Time Dependent Temperature Distribution in Radiating solids, J. Math. Phys. 39, 246-257.

Awbery, J.H. (1936). Temperature-Rise in a Material of which the Thermal Properties Vary with Temperature, Proc. Phys. Soc, 48, 118-124.

Bailey, P.B., Shampine, L.F., Waltman, P.E. (1968). Nonlinear Two Point Boundary Value Problems, Academic Press, New York and London.

Birkhoff, G., and Rota, G.-C. (1969). Ordinary Differential Equations, 2nd Ed. Blaisdel1, Waltham Massachusetts.

Boltzmann, L. (1894). Zur Integration der Diffusionsgleichung bei variabeln Diffusionscoefficienten, Ann. Physik 53, 959-964.

Carslaw, H.S., and Jaeger, J.C. (1959). Conduction of Heat in Solids, 2nd Ed. Oxford Univ. Press (Clarendon), London and New York.

Clarke, D.M. (1957). Free Diffusion: Diffusion Coefficient Varying Exponentially with Concentration, J. Chem. Phys. 27, 29-32.

Crank, J., and Henry, M.E. (1949a). Diffusion in Media with Variable Properties: I, Trans. Faraday Soc. 45, 636-642. •

Crank, J., and Henry, M.E. (1949b). Diffusion in Media with Variable Properties: II, Trans. Faraday Soc. 45, 1119-1128.

Crank, J., and Park, G.S. (1949). An Evaluation of the Diffusion Coefficient for Chloroform in Polystrene from Simple Absorption Experiments, Trans. Faraday Soc. 45, 240-249.

Friedmann, N.E. (1956). Quasilinear Heat Flow, Doctoral Dissertation, University of California, Los Angeles, Calif., November, 1956.

Friedmann, N.E. (1957). Quasilinear Heat Flow, Trans, ASME 79, 1-10.

Fujita, H. (1952a). The Exact Pattern of a Concentration-Dependent Diffusion in a Semi-infinite Medium, Part I, Text. Res. J. 22, 757-760.

Fujita, H. (1952b). The Exact Pattern of a Concentration-Dependent Diffusion in a Semi-infinite Medium, Part II, Text. Res, J. 22, 823-827.

Fujita, H. (1954). The Exact Pattern of a Concentration-Dependent Diffusion in a Semi-infinite Medium, Part III, Text. Res. J. 24, 234-240.

Goodman, T.R. (1958). The Heat-Balance Integral and Its Applications to Problems Involving a Change of Phase, Trans. ASME $\underline{80}, 335-342$. 
Goodman, T.R. (1961). The Heat-Balance Integral - Further Considerations and Refinements, J. Heat Transfer 83, 83-85.

Hopkins, M.R. (1938). Heat-Conduction in a Medium Having Thermal Properties Depending on the Temperature, Proc. Phys. Soc. 50, 703-706.

Kidder, R.E. (1957). Unsteady Flow of Gas Through a Semi-infinite Porous Medium, J. Appl. Mech. 24, 329-332.

Kirchhoff, G., and Hansemann, G. (1880). Ueber die Leitungsfähigkeit des Eisens für die Wärme, Annln. Phys, 9, 1-47.

Kirchhoff, G. (1894). Vorlesungen über die Theorie der Wärme, Barth, Leipzig.

Klute, A. (1952a). A Numerical Method for Solving the Flow Equation for Water in Unsaturated Materials, Soil Sci. 73, 105-116.

Klute, A. (1952b). Some Theoretical Aspects of the Flow of Water in Unsaturated Soils, Soiz Sci. Soc. Am. Proc. 16, 144-148.

Knight, J.H., and Philip, J.R. (1973). Solving the Flow Equation: 2, Soir Sci. (To appear).

Landahl, H.D. (1953). An approximation Method for the Solution of Diffusion and Related Problems, Bulz. Math. Biophys. 15, 49-61.

Macey, R.I. (1959). A Quasi-Steady-State Approximation for Diffusion Problems: I, Buzl. Math. Biophys. 21, 19-32.

Mann, W.R., and Wolf, R. (1951). Heat Transfer between Solids and Gases, Q. Appl. Math. $\underline{9}, 163-184$.

Park, G.S. (1950). The Diffusion of Some Halo-Methanes in Polystyrene, Trans. Faraday Soc. 46, 684-697.

Parlange, J.-Y. (1971a). Theory of Water Movement in Soils: I. Onedimensional Absorption, Soil Sci. 111, 134-137.

Parlange, J.-Y. (1971b). Theory of Water Movement in Soils: II. Onedimensional Infiltration, Soil Sci. 111, 170-174.

Parlange, J.-Y. (1971c). Theory of Water Movement in Soils: III. Two and Three Dimensional Absorption, Soil Sci. 112, 313-317.

Philip, J.R. (1954), Some Recent Advances in Hydrologic Physics, J. Inst. Engnrs. Australia 26, 255-259.

Philip, J.R. (1955a). The Concept of Diffusion Applied to Soil Water, Proc. Natz. Acad. Sci. India Sect. A 24, 93-104.

Philip, J.R. (1955b). Numerical Solution of Equations of the Diffusion Type with Diffusivity Concentration-dependent, Trans. Faraday Soc. 51, 885-892.

Philip, J.R. (1957). The theory of Infiltration 1. The infiltration equation and its solution, Soil Sci. 83, 345-357. 
Philip, J.R. (1960a). A Very General Class of Exact Solutions in Concentration-dependent Diffusion, Nature 185, 233.

Philip, J.R. (1960b). General Method of Exact Solution of the Concentration-dependent Diffusion Equation, Aust. J. Phys, 13, 1-12.

Philip, J.R. (1969). Theory of Infiltration, Adv. Hydrosci. ㅁ, 215-296.

Philip, J.R. (1973). On Solving the Unsaturated Flow Equation: 1. The flux-concentration relation, Soil Sci. (To appear).

Picard, E. (1893). Sur 1'application des méthodes d'approximations successives à l'étude de certaines équations différentielles ordinaires, J. Math. $\underline{9}, 217-271$.

Poincaré, H. (1892). Les Méthodes Nouvelles de la Mécanique Céleste I. Gauthier-Villars, Paris.

Rudin, W. (1964). Principles of Mathematical Analysis, 2nd Ed. McGrawHill, New York.

Seyferth, C. (1962). Die Eindeutigkeit von Lösungen der eindimensionalen Diffusionsgleichung mit konzentrationsabhängigem Diffusionscoeffizienten, Math. Nachr. 24, 13-32.

Storm, M.L. (1951). Heat Conduction in Simple Metals, J. Appl. Phys. 22, 940-951.

Yampol'skii, N.G., and Aizen, A.M. (1968). The Perturbation Method as Applied to Nonlinear Heat-conduction Problems, Teplofizika Vysokikh Temperatur $\underline{6}$, 885-890 (Russian). English translation in High Temp. $\underline{6}, 845-850$.

Yang, K.T. (1958). Conduction in a Semi-infinite Solid with Variable Thermal Conductivity, Trans. ASME 80, 146-147.

Williamson, J.H. (1962). Lebesgue Integration, Holt, Rinehart and Winston, New York.

Philip, J.R., and Knight, J.H. (1973). On Solving the Unsaturated Flow Equation: 2. New quasi-analytical technique, Soil Sei. (To appear). 


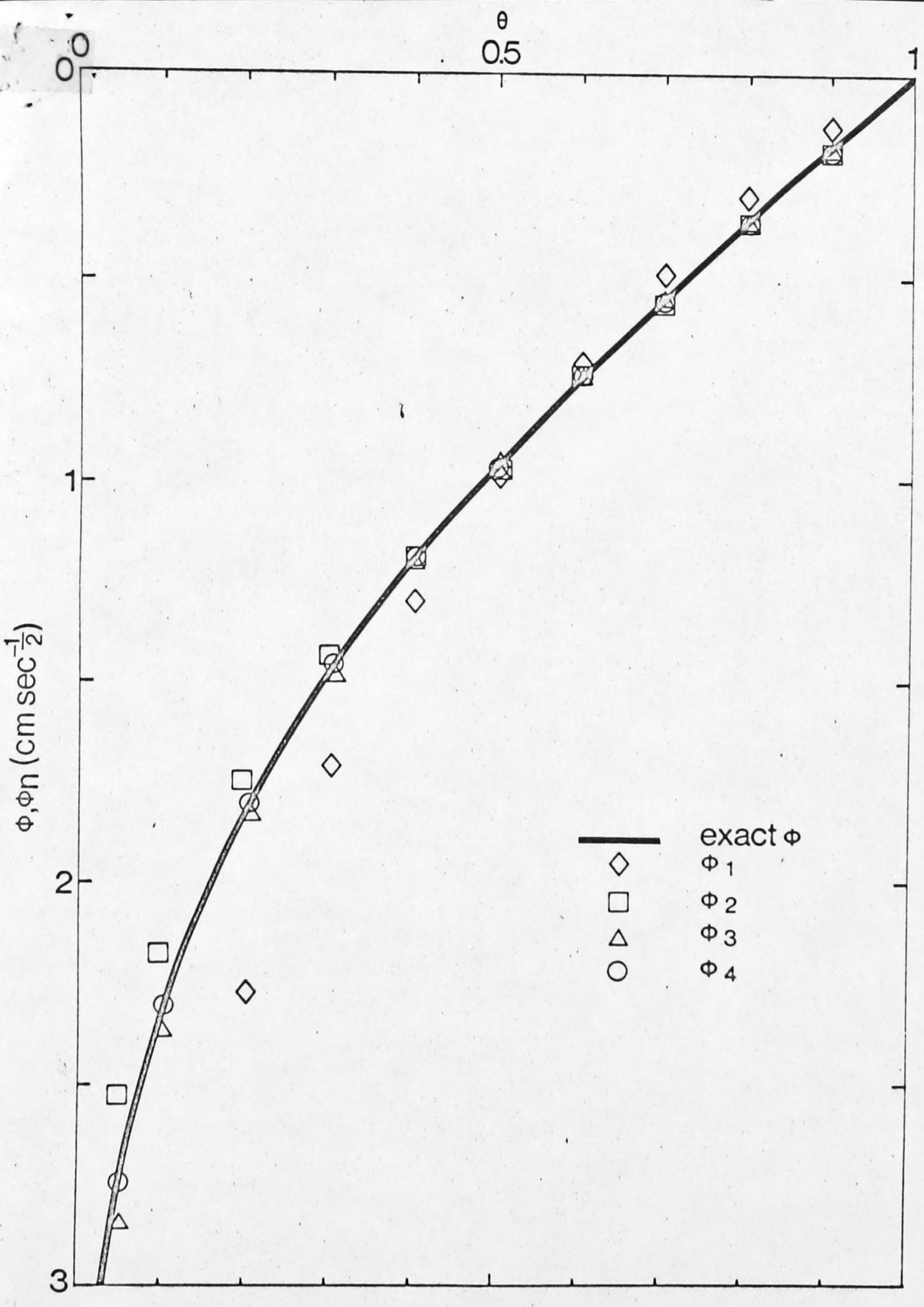

Fig.1 


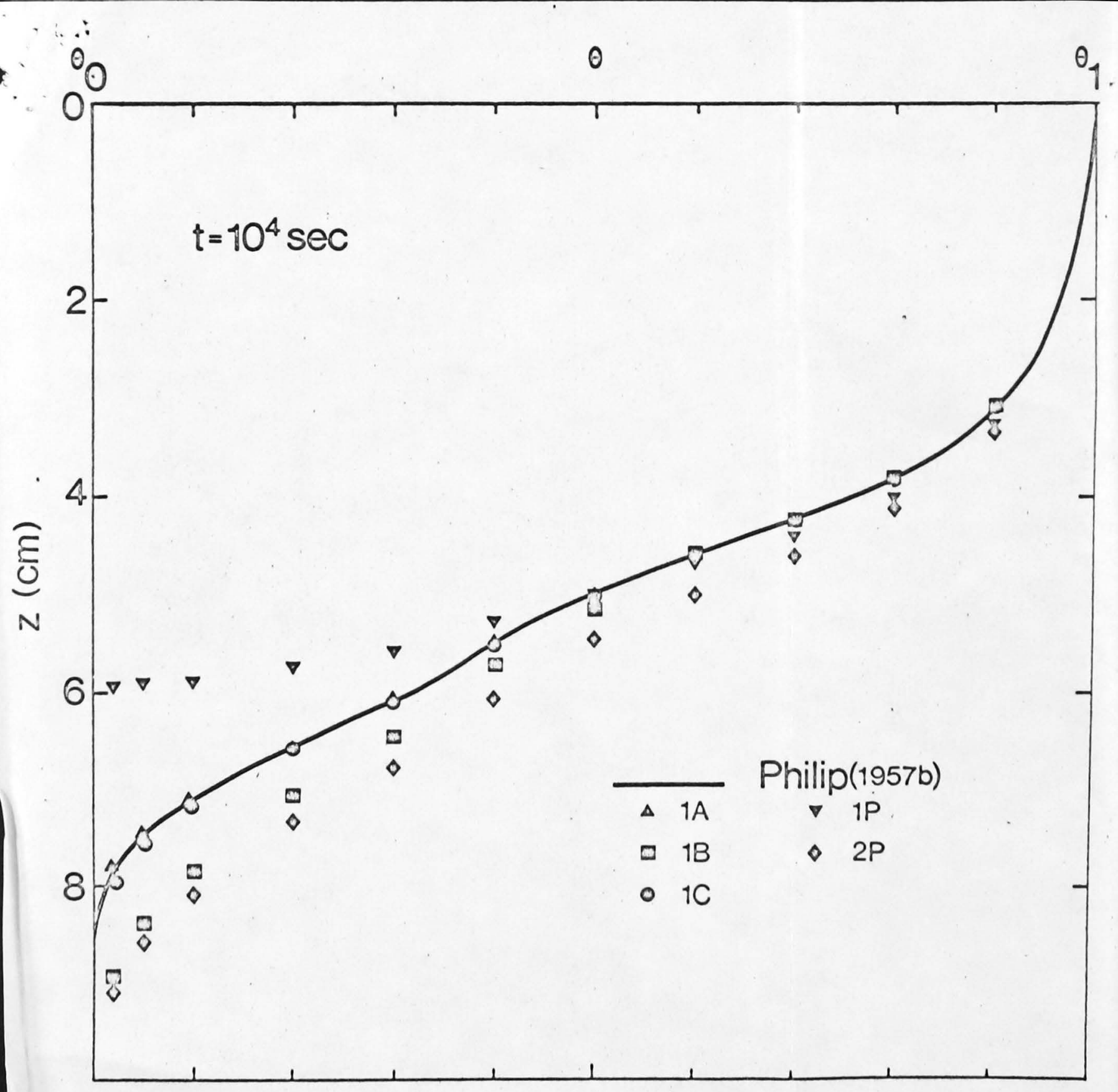

Fig.3 


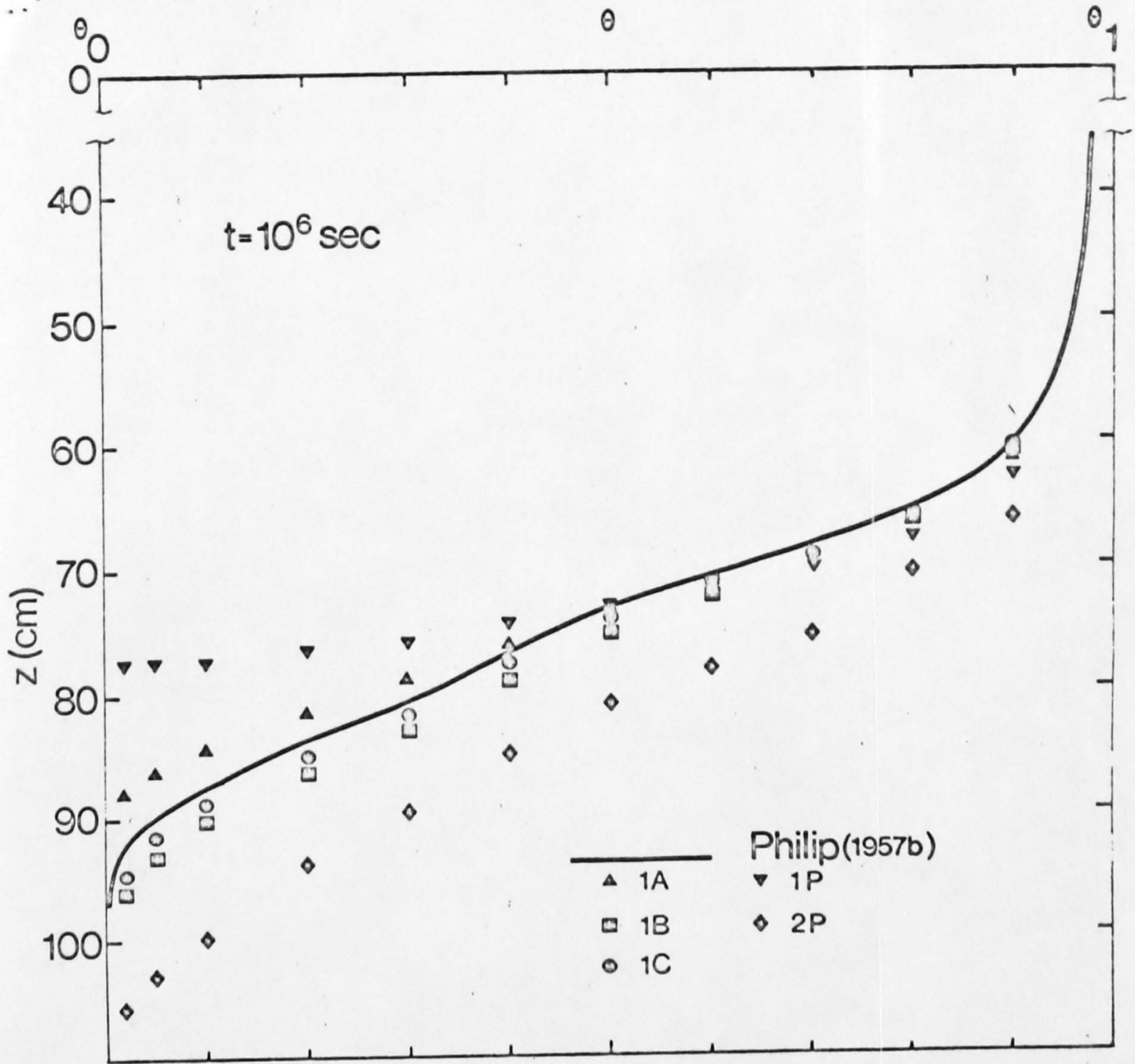

Fig.5 


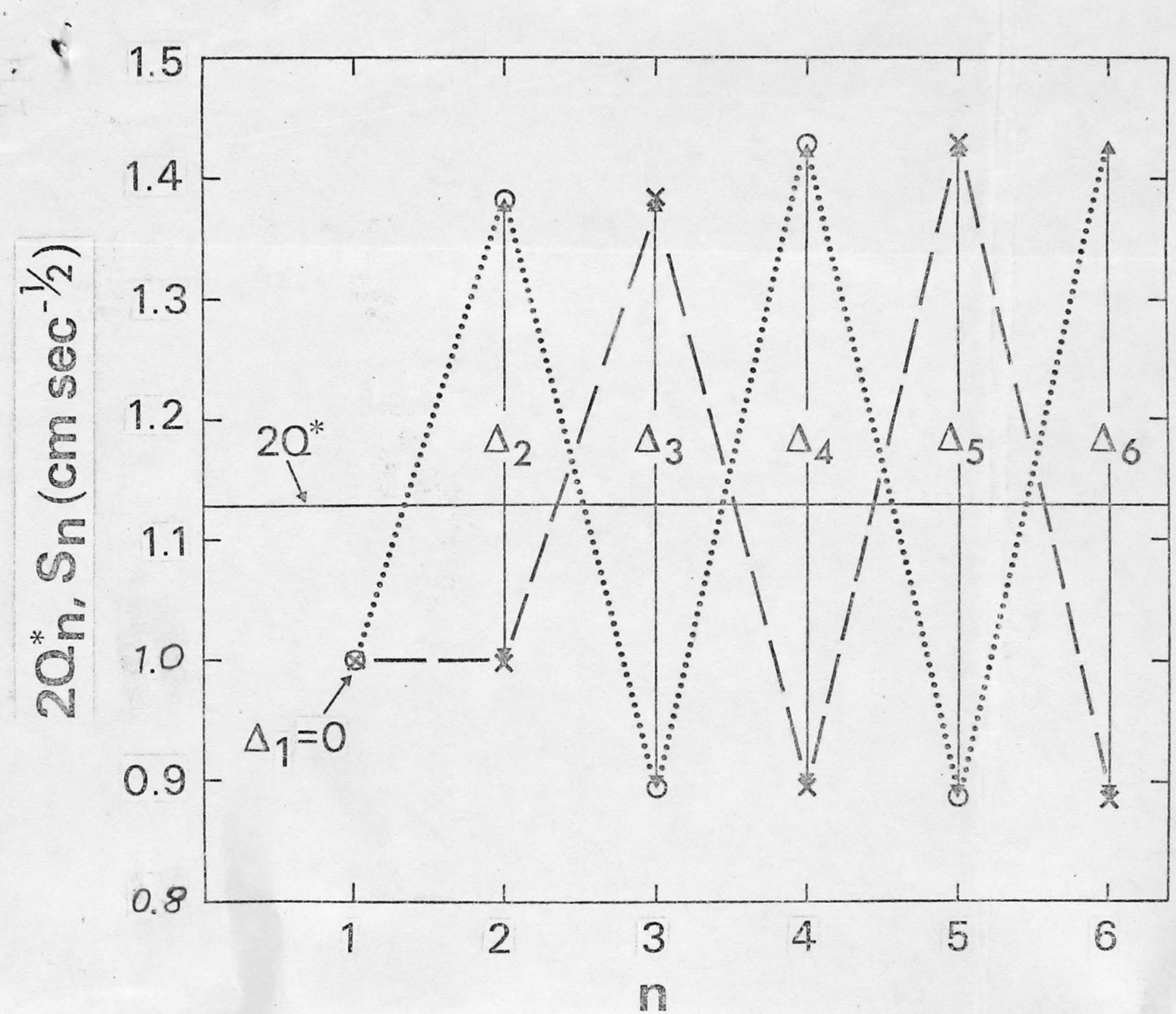

Fig. I "On Solving the Unsaturated FlowEquation : 2. Critique of Parlange's Method" by J.H. Knight and J.R. Philip. 


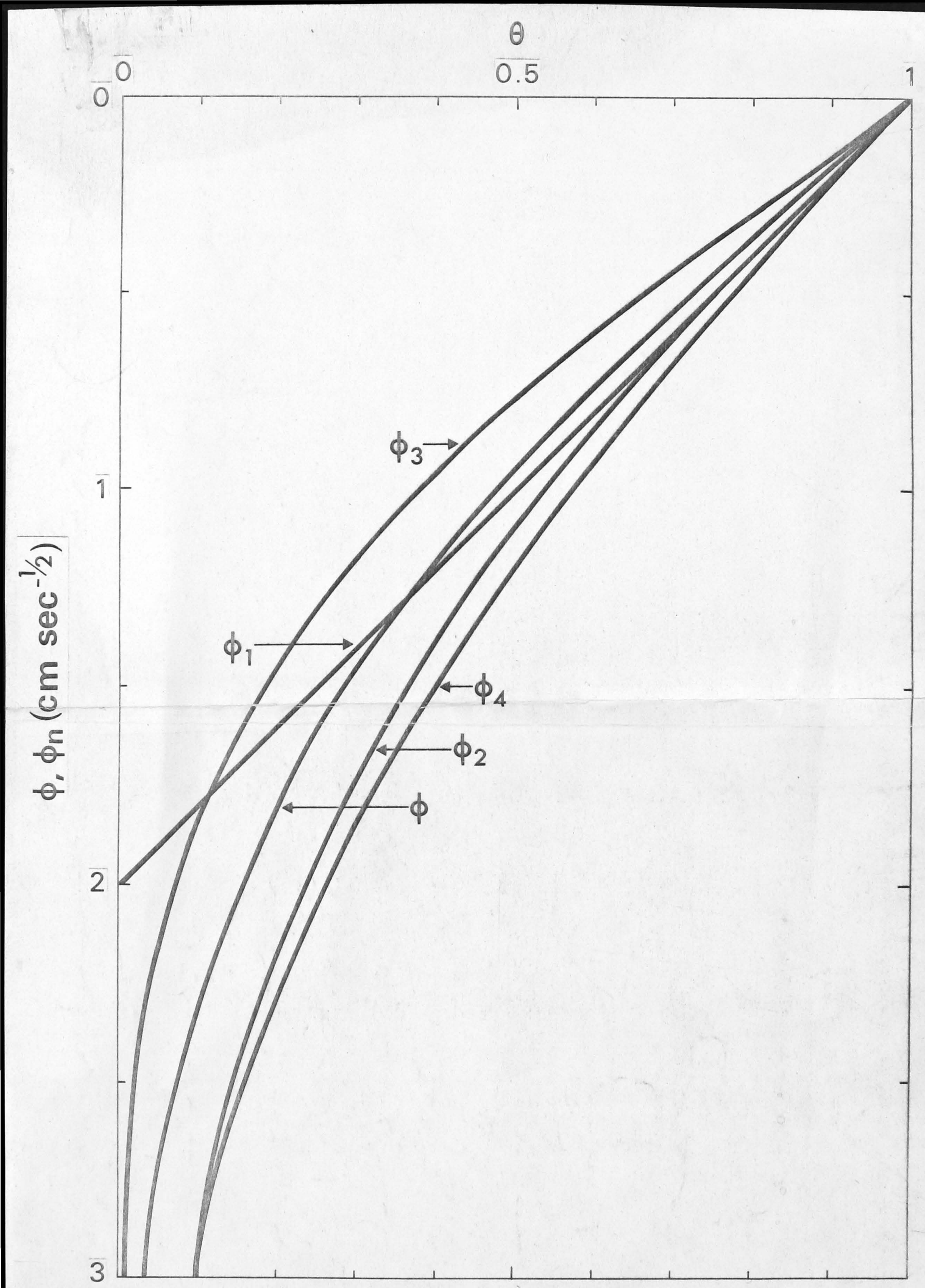
Fig. 2 "On Solving the Unsaturated Flow Equation : 2 Critique of Parlange's Method"
by J.H. Knight and J.R. Philip 


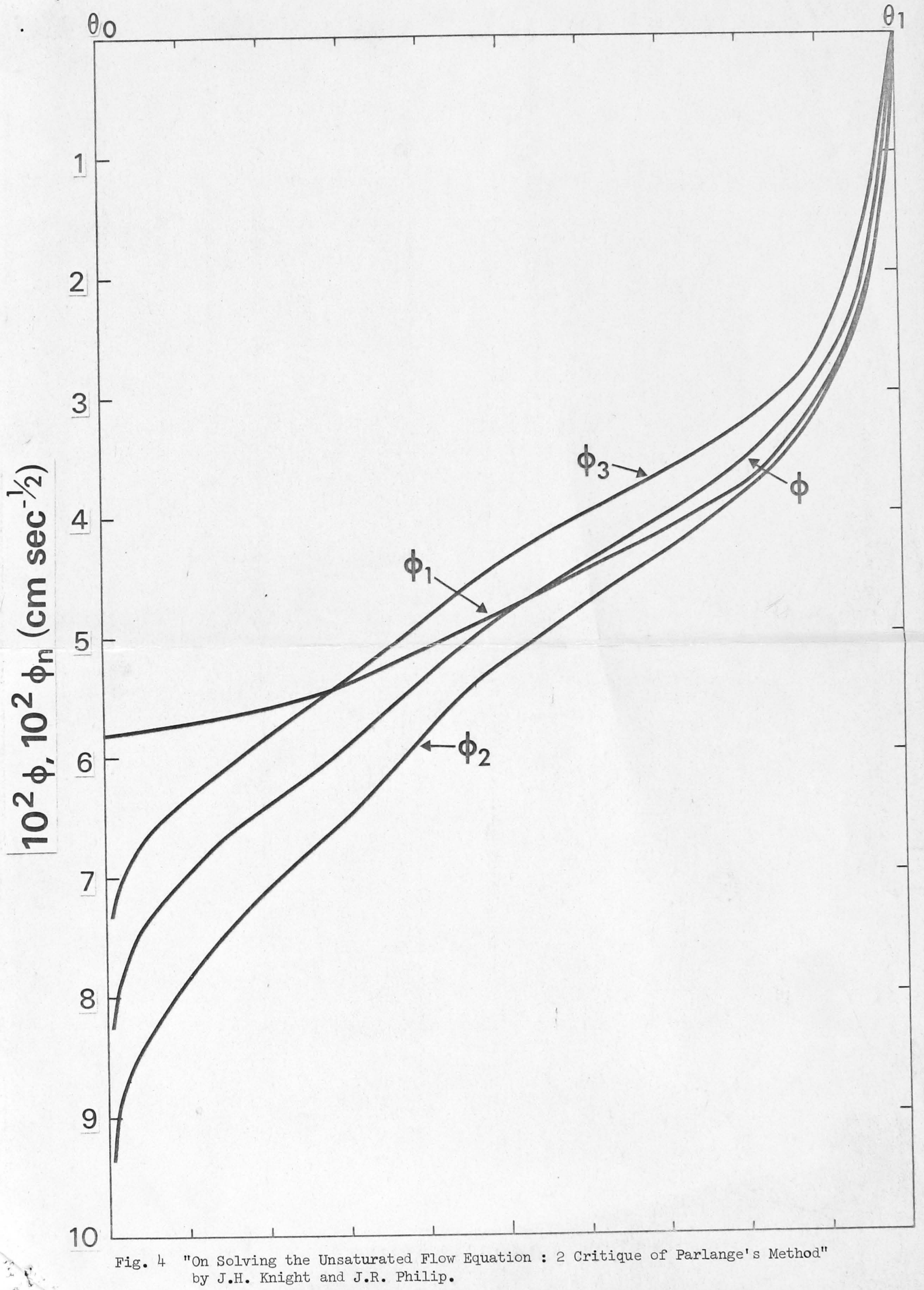




\section{Appendix 1. Detailed Calculations from Chapter 5.}

I. Constant-flux boundary condition.

Tumerical values were calculated for the

solution of the problem with zero initial concentration and constant flux imposed at the boundary, for diffusivity

$D(\theta)=(1-\theta)^{-2}$.

The initial and boundary conditions are:-

$\theta=0$ when $t=0$

$\left[D(\theta) \frac{\partial \theta}{\partial x}\right]_{x=0}=-1$

Equations (79), (80), (8I) and (82) were used

to give an implicit relationship between the concentration $\theta$ and the distance $x$ at various values of the time $t$.

Figure 1 shows the time course of the concentration-distance relation.

It can be seen that for small time, the concentration-distance relation is very similar to that obtained when the diffusivity is constant.

For larger time, the diffusion coefficient at the surface $x=0$ increases markedly as the surface concentration increases, and the diffusate is removed from the surface very rapidly. The surface concentration approaches unity, but the severity of the singularity in $D(\theta)$ ensures that the surface concentration does not attain the value unity in any finite time interval. The shape of the concentration distance relation approaches that of a pulse moving into the medium with unit velocity. This large-time behaviour is qualitatively quite different from the behaviour of the solution of a linear diffurion equation. 
II. Instantaneous source solution

Numerical values were calculated for the solution of the problem with instantaneous source of unit strength at the boundary of the semi-infinite region $x \geqslant 0$. Equations (89) and (90) were used to calculate the distance $x$ as a function of the concentration $\Theta$ at various values of the time $t$. The classical abstraction of an instantaneous point source with infinite initial concentration cannot be realized for this mode of nonlinear diffusion. The instantaneous source is required to be distributed with unit initial concentration over a region of unit size. In this region it is the initial diffusivity which is infinite, rather than the concentration.

Figure 2 shows the time course of the concentration profiles. The shape of the profile varies systematically from a step-function at zero time to a Gaussian distribution for large time. We thus have the opportunity to match initial distribution of various intermediate shapes. 
Figure 1

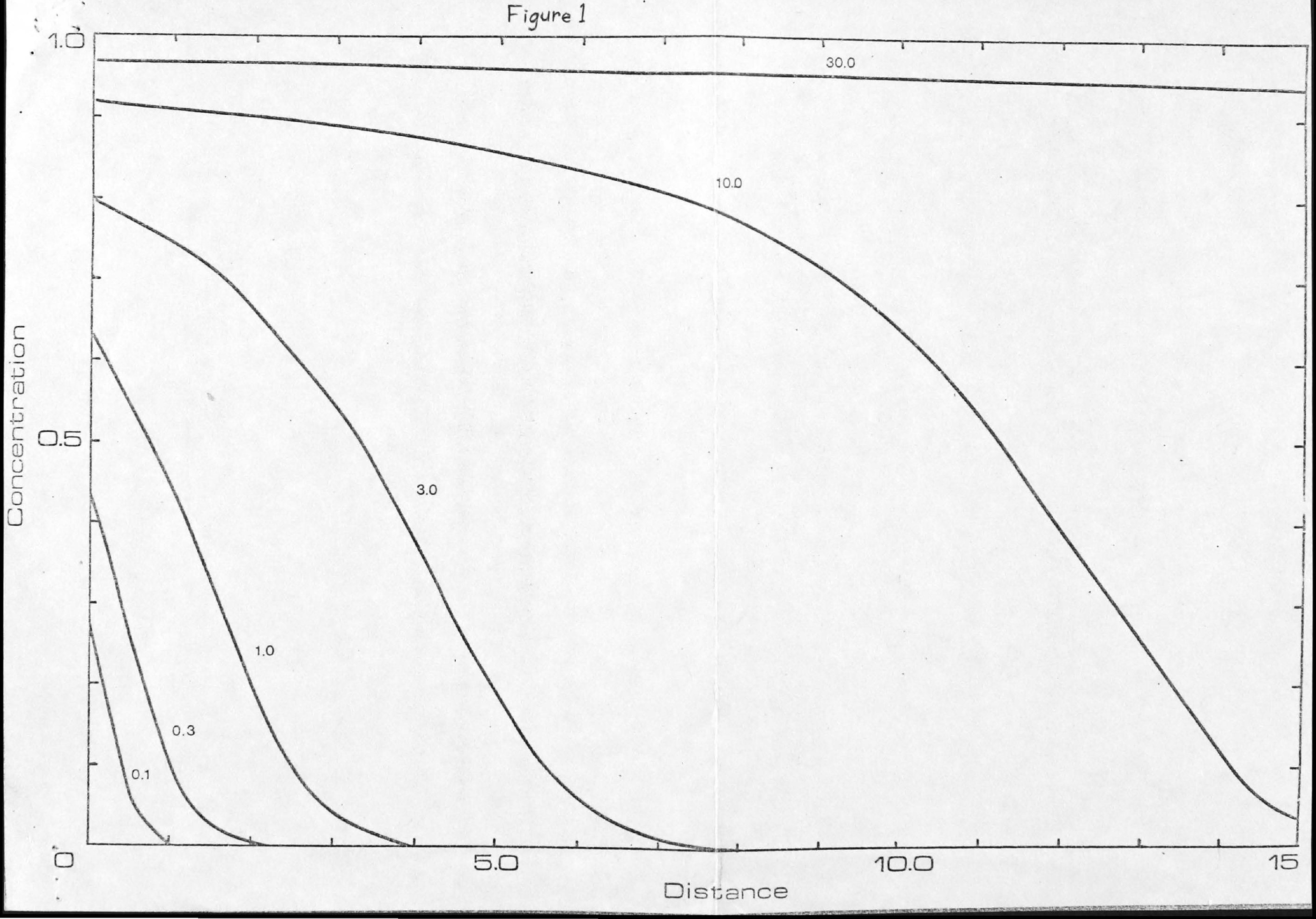




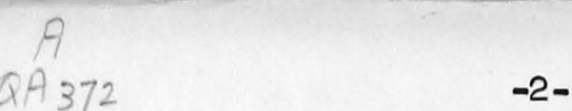

$k_{56}$

Complete Title:

ON SOLVING THE UNSATURATED FLOW EQUATION : 2.

CRITIQUE OF PARLANGE'S METHOD 
Authors' Names and Affiliations:

\section{J.H. KNIGHT}

$$
\begin{gathered}
\frac{\text { Australian National University }}{\text { and }} \\
\text { J.R. PHIIP } \\
\text { CSIRO, Australia }
\end{gathered}
$$

Received for publication .May.... 1972.

in final format November $1.2 . .1972$. 


\section{Abstract:}

The physical content of Parlange's method of solving the flow equation is explored. His first approximation, which was developed by Macey (1959), satisfies continuity in the integral sense, but the second and higher approximations do not. Approximations beyond the first lack any constraining link between the separate steps of 'satisfying continuity' and 'satisfying Darcy's law', which make up each iteration. There is, consequently, nothing in the procedure to ensure convergence.

A detailed investigation establishes the nonconvergence of the method when applied to one-dimensional sorption. It is found that the first approximation is best, and that the higher approximations make oscillations of increasing magnitude about the exact solution. Two illustrative examples are given. There is no reason to expect the procedure to be any more useful in other cases.

The utility of Parlange's method is thus simply the utility of the first approximation: the dependence of this on the shape of the diffusivity function and on the flux-concentration relation is discussed.

Contrary to Parlange's claim, the method cannot be applied to two- and three-dimensional systems other than radially symmetrical ones. 
Part 1 (Philip 1973) introduced the concept of the flux-

concentration relation in flow phenomena in unsaturated soils. This Part 2 is a critique of a method of solving the unsaturated flow equation due to Parlange (1971a). In Part 3. (Philip and Knight we develop a new technique for solving this equation and establish new quasi-analytical approximations to the solution of a range of unsaturated flow problems.

We treat various aspects of Parlange's proposed method. Firstly, Parlange (197la,b,c, 1972) does not give a clear or consistent account of the physical meaning either of his initial approximation or of the subsequent steps in his method. Accordingly, we begin by restating Parlange's method with attention to its physical content. This has the advantage, we believe, of making the significance of Parlange's work clearer to many readers; and it leads naturally to our further analysis of Parlange's work.

Secondly, and this is the most important element in our critique, we establish the failure of convergence of Parlange's method. We carry the analysis of this failure through in full detail for one-dimensional sorption with constant concentration conditions; and we discuss the question of convergence for other phenomena.

The accuracy of any approximate solution cannot exceed the accuracy with which it embodies the integral properties of the exact solution. As we show, Parlange's first approximation to the integral properties of the solution is better than the subsequent ones. The utility of the method therefore stands or 
falls on the accuracy of this first approximation. The third part of this critique is therefore a discussion of the accuracy of the first approximation.

Finally, we explain why Parlange's, and comparable, methods of solving the flow equation are necessarily restricted to systems described by one space coordinate. The only relevant two- and three-dimensional problems are radially symmetrical ones, though Parlange (1971c) states that his method applies generally to two- and three-dimensional systems 。 
We shall work with various specialized forms of the equation describing water movement in an unsaturated homogeneous nonswelling soil. These are equations (2) - (4) of Part 1, but it is convenient to restate them:

$$
\begin{gathered}
\frac{\partial \theta}{\partial t}=\frac{\partial}{\partial x}\left[D \frac{\partial \theta}{\partial x}\right] ; \\
\frac{\partial \theta}{\partial t}=\frac{\partial}{\partial z}\left[D \frac{\partial \theta}{\partial z}\right]-\frac{d K}{d \theta} \cdot \frac{\partial \theta}{\partial z} ; \\
\frac{\partial \theta}{\partial t}=r^{1-m} \frac{\partial}{\partial r}\left[D r^{m-1} \frac{\partial \theta}{\partial r}\right] .
\end{gathered}
$$

$\theta$ is the volumetric moisture content and $t$ is time. $D$, the moisture diffusivity, and $\pi$, the hydraulic conductivity, are (usually strongly-varying) functions of $\theta$ 。 Equation ( $\underline{\text { I }}$ ) applies to one-dimensional sorption, equation (2) to one-dimensional infiltration, and equation (3) to radially symmetrical mdimensional ( $m=2,3)$ sorption. Horizontal, vertical (positive downward) and radial space coordinates are denoted by $x, z$, and $x$, respectively. We are concerned with the solution of these equations subject to initial conditions (4) and one or other of boundary conditions (ㅁ) $-(\underline{8})$ :

$$
\begin{aligned}
& t=0, \theta=\theta_{0} \text { in } \mathrm{x}>0 \text { or } \mathrm{z}>0 \text { or } \mathrm{r}>\underline{r}_{0} ; \\
& t \geqq 0, \theta=\theta_{1} \text { at } \mathrm{W} ; \\
& t \geqq 0, \mathrm{D} \partial \theta / \partial \mathrm{x} \text { or } \mathrm{D} \partial \theta / \partial \mathrm{z}-\mathrm{K} \text { or } \mathrm{D} \partial \theta / \partial \mathrm{r}=-0 \text { at } \mathrm{W} ; \underline{(6)} \\
& t \geqq 0, \quad \theta=\theta_{1}(t) \text { at } \mathrm{W} ; \\
& t \geqq 0, \mathrm{D} \partial \theta / \partial \mathrm{x} \text { or } \mathrm{D} \partial \theta / \partial \mathrm{z}-\mathrm{K} \text { or } \mathrm{D} \partial \theta / \partial \mathrm{z}
\end{aligned}
$$

$$
=-Q(t) \text { at } W_{0}
$$


These are discussed more fully in Part 1 . W denotes, as required, $x=0, z=0$, or $r=r_{0}$.

\section{Equations (1) - (3) are established by combining}

Darcy's law and the continuity requirement (Klute, 1952; Philip 1957a, 1969 shall find it useful to revert to the component equations.

The specialized forms of the continuity equation, rearranged

with the space coordinate as a dependent variable (cf. Philip 1957a, 1969)

are

$$
\partial x / \partial t=\partial v / \partial \theta, \partial z / \partial t=\partial v / \partial \theta, \partial r^{m} / \partial t=m r^{m-1} \partial v / \partial \theta
$$

Integrating these with respect to $\theta$ gives

$$
v=\int_{\theta}^{\theta}(\partial x / \partial t) d \theta, v-K_{0}=\int_{\theta}^{\theta}(\partial z / \partial t) d \theta, m r^{m-1} v=\int_{\theta}^{\theta}\left(\partial r \frac{m}{\theta^{m}} / \partial t\right) d \theta
$$

We write $K_{0}$ for $K\left(\theta_{0}\right)$. With $\theta=\theta_{1}$ these become

$Q=\int_{\theta_{0}}^{\theta_{1}}(\partial x / \partial t) d \theta, Q-K_{0}=\int_{\theta_{0}}^{\theta_{1}}-(\partial z / \partial t) d \theta, m r_{0}{ }^{m-1} Q=\int_{\theta_{0}}^{\theta_{1}}\left(\partial r^{m} / \partial t\right) d \theta_{0}$

We also introduce the relevant specialized forms of Darcy's law:

$$
\mathrm{v}=-\mathrm{D} \partial \theta / \partial \mathrm{x}, \mathrm{v}=\mathrm{K}-\mathrm{D} \partial \theta / \partial \mathrm{z}, \mathrm{v}=-\mathrm{D} \partial \theta / \partial \mathrm{r}
$$

The flux-concentration relation $F(\Theta)$ defined by

$$
\Theta=\frac{\theta-\theta_{0}}{\theta_{1}-\theta_{0}}, \quad F=\frac{v}{Q} \text { or } \frac{v-K_{0}}{Q-K_{0}} \text { or }\left(\frac{r}{r_{0}}\right)^{m-1} \cdot \frac{v}{Q}
$$

was introduced in Part 1 . It evidently follows from equations (10) and (11) that 

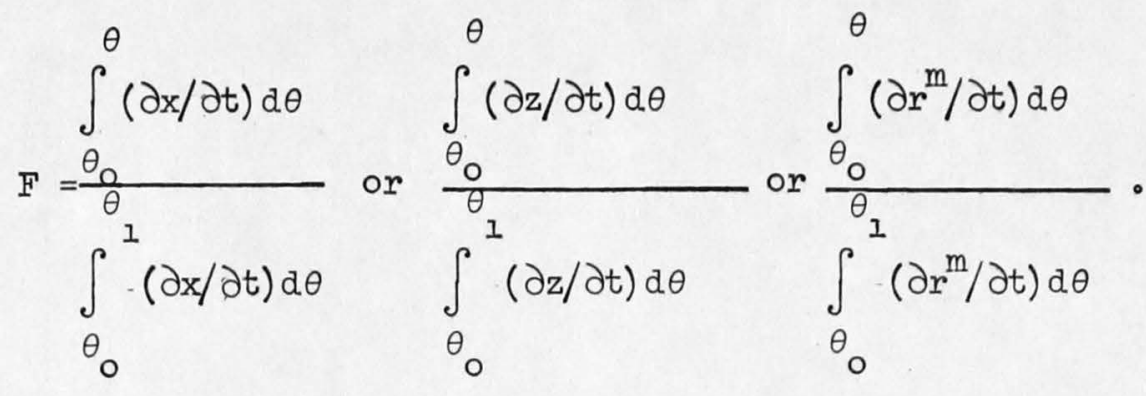

PARLANGE'S PROCEDURE AND ITS PHYSICAL CONTENT

Rather than attempt a summary of Parlange's own presentation of his method, we prefer to offer a simple and direct explanation with close reference to its physical content. We first discuss the solution of equations (1) - (2) subject to concentration conditions (4), (5) or (4), (7) and we shall then treat solutions satisfying flux conditions.

\section{Solutions subject to concentration conditions}

The first approximation. Parlange's first step is exactly equivalent to assuming that

$$
\Theta=0, F=0 ; 0<\Theta \leqq 1, F=1 .
$$

This is precisely relation (19) of Part 1. Physically ${ }^{4}$ it means that, everywhere in the region influenced by the change in conditions at $\mathrm{W}$, the total flow across any surface $\mathrm{y}=$ constant is taken as constant and equal to that across $\mathrm{W}$. We use y here, and in what follows, to denote the appropriate space coordinate, that is $\mathrm{x}$ or $\mathrm{z}$ or $r$. Putting $\mathrm{Q}$, then, for $\mathrm{v}\left[\mathrm{or}\left(\mathrm{r} / \mathrm{r}_{0}\right)^{\mathrm{m}-1} \mathrm{v}\right]$ in the relevant Darcy equation (12) and integrating yields a first estimate of $\mathrm{y}(\theta)$ for given $\mathrm{Q}, \mathrm{y}_{1}$ (say). Putting $\mathrm{y}=\mathrm{y}_{1}$ in the relevant equation (그) leads to a first-order differential equation which may be solved to give a first estimate of $Q$ as 
a function of $t, Q_{I}$ (say). Physically, this use of equation

(11) amounts to requiring thatcontinuity be satisfied in the integral sense. Combining $Q_{1}(t)$ and $y_{2}\left(\theta, Q_{1}\right)$ yields the first approximation $\mathrm{y}_{1}(\theta, t)$. In special cases $\mathrm{y}_{1}(\theta, t)$ may be expressed explicitly; in general, however, it must be left in implicit form. It should be noted that this first approximation wa developed and used by Macey (1959).

The second approximation. Parlange's second approximation consists of putting $\mathrm{y}_{1}$ for $\mathrm{y}$ in the appropriate continuity equation (10) to obtain a second estimate of $\mathrm{v}, \mathrm{v}_{2}$; and of then putting $\mathrm{v}=\mathrm{v}_{2}$ in the relevant Darcy equation (12) and integrating to secure a second estimate of $\mathrm{y}(\theta, t), \mathrm{y}_{2}$. In view of equation $(\underline{I I}), v_{2}\left(\theta_{I}\right)=Q_{2}=Q_{1}$, so that the second approximation leaves $Q$ unchanged.

We observe the peculiarity that, in this second approximation, Parlange has abandoned the continuity requirement, even in the integral sense. The value of $Q$, and therefore of $\partial \theta / \partial y$ at $w$, is inconsistent with the value of $\int_{\theta_{I}}^{\theta_{1}}(\partial y / \partial t) d \theta$ [or of $\left.\int_{\theta_{0}}^{\theta_{1}}\left(\partial r^{m} / \partial t\right) d \theta\right]$. There is no a priori reason to expect this second approximation to be an improvement on the first, which at least satisfied an integral continuity constraint. As we shall show, the intuition that feeding the first estimate back through equations $(\underline{10})$ and (12) ought to improve matters cannot, in fact, be sustained.

The third and higher approximations. Parlange states that the third and higher approximations may be developed through 
an iterative scheme following the pattern of the second approximation. The third approximation, then, involves putting $\mathrm{y}_{2}$ for $\mathrm{y}$ in the appropriate equation (10) to secure the third estimate of $\mathrm{v}, \mathrm{v}_{3}$. Unlike the second approximation, this one leads to a fresh estimate of $Q$, namely $Q_{3}(t)=v_{3}\left(\theta_{1}, t\right)$. Putting $v=v_{3}$ in the appropriate equation (12) and integration then gives the third estimate of $\mathrm{y}, \mathrm{y}_{3}$. Combining $\mathrm{Q}_{3}(t)$ and $\mathrm{y}_{3}(\theta, Q)$ yields the third approximation $\mathrm{y}_{3}(\theta, t)$. Parlange proposed iterating this scheme to obtain higher approximations, in the expectation that the procedure is convergent and provides a sequence of improving estimates of the solution. None of these estimates satisfy continuity, even in the integral sense. The approximations beyond the first lack any constraining link between the two separate steps of 'satisfying continuity' and 'satisfying Darcy's law', which make up a single iteration. Nothing in the procedure ensures that both will be ever satisfied simultaneously, or even that initial discrepancies will be reduced. These considerations raise doubts about the convergence of Parlange's method. We turn to this question in the following section.

\section{Solutions subject to flux conditions}

Parlange (1972) has applied his method to solutions subject to constant flux conditions (ㄴ),$(\underline{6})$, and variable flux conditions (4) , ( 9 ) may also be considered. In these var cases, $Q(t)$ is given and we seek $\theta_{1}(t)$ as part of the required solution. 
The first approximation again involves assumption of relation (15), integration of equation (12) and the use of (II), which, in this case, yields an estimate of $\theta_{1}(t)$. As before, the first approximation satisfies continuity in the integral sense.

The second approximation again consists of putting $\mathrm{y}_{1}$ for $\mathrm{y}$ in equation (10) and integrating equation (12) to obtain a second estimate of $y, y_{2}(\theta, t)$. In this case, because of equation (ㄱ) , the second approximation leaves the estimate of $\theta_{1}(t)$ unchanged. As before, this second approximation fails to satisfy continuity, even in the integral sense.

For this class of problem, Parlange has not specifically proposed higher approximations. His iterative scheme for problems subject to concentration conditions cannot carry over without some modification.

\section{FAILURE OF CONVERGENCE OF THE METHOD}

One-dimensional sorption subject to constant concertration conditions

It has been emphasized (Philip 1969 and Part 1) that the solution of equation ( 1 ) subject to conditions (ㅁ), (ㅁ) is central to the study of solutions of the unsaturated flow equation. The appropriate point of departure for our examination of the convergence of Parlange's method is this solution.

Parlange (197la) treated one-dimensional sorption in terms of (in our symbolism) $x, t$ and $-Q^{-1}$. Our use of his method here 
differs superficially in that we simplify the presentation by working with the reduced quantities

$$
\phi=x t^{-\frac{1}{2}}, V=v t^{\frac{1}{2}}, Q^{*}=Q t^{\frac{1}{2}} \text {. }
$$

These apply because of the well-known (Klute 1951; Philip 1955, 1969) character of the solution. The convergence of the method is, of course, left unaffected by our choice of variables. We note that $2 Q^{*}=S$, where $S$, the sorptivity (Philip 1957b, 1969) defined as $\int_{\theta_{0}^{-}}^{\theta_{1}} \phi d \theta$. We shall see that, in the first approximation, which preserves integral continuity, $2 Q_{I}^{*}=S_{I}$ [equation (18)]; but that, for Parlange's second-and higher approximations, where integral continuity has been abandoned, $2 Q_{n}^{*}$ is altermately less than ( $n$ even) and greater than ( $n$ odd) $\mathrm{s}_{\mathrm{n}}$ 。

Parlange's first approximation. Use of relation (15) in the first of equations (12) then yields

$$
Q_{1}^{*}=-D d \theta / d \phi_{I} \text {, i.e. } \phi_{I}=\left(Q_{I}^{*}\right)^{-I} \int_{\theta}^{\theta^{I}}-D d \theta
$$

and the first of equations (그) reduces to

$$
Q_{1}^{*}=\frac{1}{2} \int_{\theta_{0}}^{\theta_{1}}-\phi_{1} d \theta=\frac{1}{2} S_{1} \text {. }
$$

Combining equations (파) and (18) gives

$$
Q_{1}^{*}=\left[\frac{1}{2} \int_{\theta_{0}}^{1}-\left(\theta-\theta_{0}\right) D d \theta\right]^{\frac{1}{2}}
$$


and

$$
\phi_{I}=\left[\frac{1}{2} \int_{\theta_{0}}^{\theta_{1}}\left(\theta-\theta_{0}\right) D d \theta\right]^{-\frac{1}{2}} \cdot \int_{\theta}^{\theta_{1}} D d \theta .
$$

The positive values of the square roots hold for absorption $\left(\theta_{1}>\theta_{0}\right)$ and the negative ones for desorption $\left(\theta_{1}<\theta_{0}\right)$. These approximations should be compared-with the exact results

$$
\begin{gathered}
Q^{*}=\left[\frac{1}{2} \int_{\theta_{0}}^{\theta^{I}\left(\theta-\theta_{0}\right) D} \frac{1}{F} d \theta\right]^{\frac{1}{2}}, \\
\phi=\left[\frac{1}{2} \int_{\theta_{0}}^{\theta^{I}} \frac{\left(\theta-\theta_{0}\right) D}{F} d \theta\right]^{-\frac{1}{2}} \cdot \int_{\theta}^{\theta^{I}} \frac{D}{F} d \theta,
\end{gathered}
$$

which are found when $Q_{1}^{*}$ is replaced in equation (17) by the exact value of $\mathrm{V}$, namely $\mathrm{FQ}^{*}$, and in equation $\left(\underline{18)}\right.$ by $Q^{*}$.

We have shown in Part 1 that (with the trivial and degenerate exception of desorption in the 'delta-function' soil)

$$
F<1 \text { for } 0<\Theta<1 \text {. }
$$

It follows therefore that

$$
\left|\int_{\theta_{0}}^{\theta^{1}}\left(\theta-\theta_{0}\right) D d \theta\right|<\left|\int_{\theta_{0}}^{1} \frac{\left(\theta-\theta_{0}\right) D}{F} d \theta\right|,
$$

so that, except in the limiting case of desorption in the 'delta-function' soil (which need not detain us), division of equation (19) by equation (리) yields 
27.

$$
\frac{Q_{1}^{*}}{Q^{*}}=\left[\frac{\int_{0}^{\theta_{1}}\left(\theta-\theta_{0}\right) D d \theta}{\theta_{1}^{\theta_{1}} \frac{\left(\theta-\theta_{0}\right) D}{F} d \theta}\right]_{\theta_{0}}^{\frac{1}{2}}=\alpha_{1}<1 .
$$

Differentiating equations (20) and (2) with respect to $\theta$ and dividing the first result by the second, we obtain

$$
\mathrm{d} \phi / \mathrm{d} \phi=\alpha_{I}^{-1} \mathrm{~F}
$$

Now $F$ is a monotonic function of $\theta$ which decreases from the value $I$ at $\theta_{I}$ to the value 0 at $\theta_{0}$ (Part $I$ ). Therefore $\mathrm{d} \phi_{1} / \mathrm{d} \phi$ is a monotonic function of $\theta$ decreasing from the value $\alpha_{1}^{-1}$, which is greater than 1 at $\theta=\theta_{1}$ to the value 0 at $\theta_{0}$. In addition, since [equation (11)]

$$
Q^{*}=\frac{1}{2} \int_{\theta_{0}}^{\theta} \phi d \theta
$$

equations (18) and (23) imply that

$$
\left|\int_{\theta_{0}^{1}}^{\theta_{1}} d \theta\right|<\left|\int_{\theta_{0}^{I}}^{\theta_{I}} \phi d \theta\right| .
$$

These properties of $\phi_{I}$ and $\phi$ imply that there exists a definite $\theta$ range $\theta_{I} \gtrless \theta \gtrless \theta^{\prime} \gtrless \theta_{0}$ such that

$$
\theta_{1} \gtrless \theta \gtrless \theta^{\prime}, \phi_{1}>\phi, \theta^{\prime} \geqslant \theta \geqslant \theta_{0}, \phi_{1}<\phi_{0}^{-}
$$


To summarize, the first approximation gives $\left|Q_{I}^{*}\right|$ smaller than the exact value $|Q|$, gives $\phi_{I}$ greater than the exact $\phi$ in the definite open interval $\theta_{1}$ to $\theta^{\prime}$, and gives $\phi_{1}$ smaller than $\phi$ in the open interval $\theta^{\prime}$ to $\theta_{0}$. Compare Tables 1 and 2 and Figures $1-4$.

Some general results for Parlange's higher approximations。 Each higher approximation consists of the two steps,

$$
\left.V_{n}=\frac{1}{2} \int_{\theta_{0}}^{\theta} \phi_{n-1} d \theta \quad \text { [based on equation (10) }\right]
$$

and

$$
\phi_{n}=\int_{\theta}^{\theta}-\frac{D}{V_{n}} d \theta[\text { integration of equation (12) }] \text {. }
$$

Performed in sequence, these make up the iterative scheme

$$
\phi_{n}=2 \int_{\theta}^{\theta_{1}} \frac{D}{\left[\int_{\theta_{0}}^{\theta} \phi_{n-1} d \theta\right]} d \theta \text {. }
$$

Implicit in the procedure is the iterative scheme for $\mathrm{V}$

$$
v_{n}=\frac{1}{2} \int_{\theta_{0}}^{\theta}\left[\int_{\theta}^{\theta} \frac{D}{V_{n-1}} d \theta\right] d \theta
$$

obtained by writing $(n-1)$ for $n$ in equation (26) and putting the resulting expression for $\phi_{n-2}$ in equation (25). With $F_{n} Q_{n}^{*}$ for $V_{n}$, this becomes

$$
2 F_{n} Q_{n-1}^{*} Q_{n}^{*}=\int_{\theta}^{\theta}\left[\int_{\theta}^{\theta_{1}} \frac{D d \theta}{F_{n-1}}\right] d \theta .
$$


Putting $\theta=\theta_{1}$ and remembering that $F_{n}\left(\theta_{1}\right)=1$, we obtain

$$
2 Q_{n-1}^{*} Q_{n}^{*}=\int_{\theta_{0}}^{\theta_{1}} \frac{\left(\theta-\theta_{0}\right) D}{F_{n-1}} d \theta_{0}
$$

Turning to the exact solution, we note that equation (I) subject to conditions (4), () reduces to the integrodifferential equation $(6,9)$

$$
-2 D \frac{d \theta}{d \phi}=\int_{\theta}^{\theta} \phi d \theta \text {. }
$$

We may rewrite this as

$$
\mathrm{V}=\frac{1}{2} \int_{\theta}^{\theta}\left[\int_{\theta}^{\theta} \cdot \frac{\mathrm{D}}{\mathrm{V}} \mathrm{d} \theta\right] \mathrm{d} \theta
$$

since $V=-D d \theta / d \phi$ and $\phi=\int_{\theta}^{I} \frac{D}{V} d \theta$. With $V=F Q^{*}$ this

becomes

$$
2 F\left(Q^{* 2}\right)=\int_{\theta}^{\theta}\left[\int_{\theta}^{\theta_{1}} \frac{D}{F} d \theta\right] d \theta,
$$

and putting $\theta=\theta_{I}$ gives the square of equation (2I). Dividing equation (29) by the square of equation

(21) yields

$$
\frac{Q_{n-I^{*} Q_{n}^{*}}^{*}}{\left(Q^{*}\right)^{2}}=\frac{\int_{0}^{\theta_{I}} \frac{\frac{\left.\theta_{0}-\theta_{0}\right) D}{F-1}}{\int_{\theta_{0}}^{\theta_{I}\left(\theta-\theta_{0}\right) D}} d \theta}{F} d \theta
$$


Differentiating equations $\underline{(28)}$ and (30) with respect to $\theta$ and dividing the first result by the second gives the relation

$$
\frac{d F_{n}}{d F}=\frac{\int_{\theta}^{\theta} \frac{F}{F_{n-1}} d G(\theta) / \int_{\theta}^{\theta} \operatorname{dg}(\theta)}{Q_{n-1}^{*} Q_{n}^{*} /\left(Q^{*}\right)^{2}},
$$

where $G=\int \frac{D}{F} d \theta$.

Finally, differentiating equation (6) with respect to $\theta$ and dividing by the equality $d \phi / d \theta=D / V$, we obtain the relation between $\phi_{n}$ and $\phi$,

$$
\mathrm{d}_{\mathrm{n}} / \mathrm{d} \phi=\mathrm{V} / \mathrm{V}_{\mathrm{n}}=\left(\mathrm{Q}^{*} / \mathrm{Q}_{\mathrm{n}}^{*}\right)\left(\mathrm{F} / \mathrm{F}_{\mathrm{n}}\right) \text { 。 }
$$

\section{Parlange's second approximation. Putting $\mathrm{n}=2$ in equation}

(31), remembering that $F_{1}=1$ on $0<\Theta \leqq 1$, and comparing the result with equation (23), we have

$$
\frac{Q_{2}^{*}}{Q^{*}}=\frac{Q_{1}^{*}}{Q^{*}}=\alpha_{1}<1 \text {. }
$$

This agrees, as it should, with the general result noted in the previous section that $Q_{2}=Q_{1}$.

$$
\text { Putting } n=2 \text { in equation ( } \underline{32} \text { ), and using ( } \underline{34} \text {, we }
$$

obtain

$$
\frac{d F_{2}}{d F}=\alpha_{I}-2 \int_{\theta}^{\theta_{I}} \frac{F_{I}}{F_{I}} d G(\theta) / \int_{\theta}^{\theta^{I}}-d G(\theta) \text {. }
$$

Now $F / F_{I}$ is a monotonic function of $\theta$ which is positive and 
less than $I$ in the open interval $\theta_{0}$ to $\theta_{1}$ and has the value 1 at $\theta_{1}$. Therefore the ratio of integrals in equation (35) is also positive and less than $I$ in the same interval and approaches the value $I$ in the limit $\theta \rightarrow \theta_{I}$. Accordingly $\mathrm{dF}_{2} / \mathrm{dF}$ has the value $\alpha_{1}^{-2}$, which is greater than 1 at $\theta=\theta_{1}$ and decreases monotonically as $\theta$ varies from $\theta_{1}$ towards $\theta_{0}$, but remains positive. Now, since $F_{2}\left(\theta_{1}\right)=F\left(\theta_{1}\right)=I$ and $F_{2}\left(\theta_{0}\right)=F_{1}\left(\theta_{0}\right)=0$, this behavior of $d F_{2} / d F$ requires that

$$
0<\Theta<1, \quad F_{2}<F
$$

and also that $F / F_{2}$ be monotonic in $0<\Theta \leqq 1$.

$$
\text { Combining equations (33) and (34) and using }
$$
inequality $(\underline{36})$, we see that

$$
\theta_{1} \lessgtr \theta \lessgtr \theta_{0}, \quad \alpha_{1} d_{2} / d \phi=F / F{ }_{2}>1 \text {, and hence } \phi_{2}>\alpha_{1}^{-1} \phi_{\cdot} \text { (37) }
$$

Also, putting both $n=I$ and $n=2$ in equation (33), we obtain

$$
\theta_{1} \lesseqgtr \theta \lesseqgtr \theta_{0}, \mathrm{~d} \phi_{2} / \mathrm{d} \phi_{1}=\mathrm{F}_{2}^{-1}>1 \text {, and hence } \phi_{2}>\phi_{1} \cdot
$$

To summarize then, the second approximation gives $\left|Q_{2}^{*}\right|$ equal to $\left|Q_{I}^{*}\right|$ and smaller than $\left|Q^{*}\right|$, and gives $\phi_{2}$ greater than either $\phi_{I}$ or $\phi$ (except, of course, at $\theta=\theta_{I}$ where $\phi_{2}=\phi_{1}=\phi=0$ ). Compåre Tables 1 and 2 and Figures $1-4$.

Parlange's third approximation. The properties of the third approximation follow similarly。 Equation (31) gives 


$$
\frac{Q_{2}^{*} Q_{3}^{*}}{\left(Q^{*}\right)^{2}}=\frac{\int_{0}^{\theta^{1}} \frac{\left(\theta-\theta_{0}\right) D}{F_{2}}}{\int_{\theta_{0}}^{1} \frac{\left(\theta-\theta_{0}\right) D}{F}} d \theta=\alpha_{3}>1,
$$

the inequality holding because $\mathrm{F}_{2}<\mathrm{F}$ [equation (36)]. Combining equations (푸) and (34) yields

$$
\frac{Q^{*}}{Q^{*}}=\alpha_{3} \frac{Q^{*}}{Q_{2}^{*}}=\alpha_{1}^{-1} \alpha_{3}>1 \text {. }
$$

We see that the magnitude of the fractional error in $Q_{3}^{*}$ is greater than that in $Q_{2}^{*}$.

We now employ equations (2) and (마), and an argument similar to that used above, to show that

$$
0<\Theta<I, \quad F_{3}>F
$$

and also that $\mathrm{F} / \mathrm{F}_{3}$ is monotonic in $0<\Theta \leqq 1$. Here $d F_{3} / d F$ is less than $I$ at $\theta=\theta_{1}$ and increases monotonically as $\theta$ varies from $\theta_{I}$ towards $\theta_{0}$.

It then follows from equation ( $\underline{33}$ ) and $(\underline{40})$ and inequality (4I) that

$$
\phi_{3} / \phi<\alpha_{1} \alpha_{3}^{-1}<1
$$

except at $\theta=\theta_{1}$ where $\phi_{3}=\dot{\phi}=0$ 。

To summarize, then, the third approximation gives $\left|Q_{3}{ }^{*}\right|$ larger than $\left|Q^{*}\right|$ and a worse approximation to it than $\left|Q_{2}{ }^{*}\right|$; and it gives $\phi_{3}$ less than $\phi_{0} \mathrm{Cf}$. Tables $I$ and 2 and Figures 1-4. 
Parlange's higher approximations. We repeat this mode of analysis for each higher approximation. The salient results develop sequentially:

$$
\begin{aligned}
& \phi / \phi_{4}<Q_{4}^{*} / Q^{*}=\alpha_{13} \alpha_{4}^{-1} \alpha_{4}<I \text {, with } \alpha_{4}<I ; F_{4}<F ; \\
& \phi / \phi_{5}>Q_{5}^{*} / Q^{*}=\alpha_{1}^{-1} \alpha_{34} \alpha_{5}^{-1} \alpha_{5}>1 \text {, with } \alpha_{5}>1 ; F_{5}>F ; \\
& \phi / \phi_{6}<Q_{6}^{*} / Q^{*}=\alpha_{13} \alpha_{45}^{-1} \alpha_{6} \alpha^{-1} \alpha_{6}<I \text {, with } \alpha_{6}<I ; F_{6}<F ;
\end{aligned}
$$

and so on. The inequalities for $\phi_{n}$ and $F_{n}$ given here and below hold for the open $\theta$ interval $\theta_{1}$ to $\theta_{0}$. It will be noted that

$$
\mathrm{n}=3,4,5,6, \ldots, \alpha_{\mathrm{n}}=\int_{\theta_{0}}^{\theta^{1}\left(\theta-\theta_{0}\right) D} \frac{\mathrm{D} d \theta}{\mathrm{F}_{\mathrm{n}-1}} / \int_{\theta_{0}}^{\theta_{1}} \frac{-\left(\theta-\theta_{0}\right) D d \theta}{\mathrm{F}}
$$

We express the various results in general form, discriminating between the cases of $n$ odd and $n$ even:

$$
\begin{gathered}
m=2,3,4,5, \ldots, \alpha_{2 m-1}>1, \alpha_{2 m}<1 ; \\
\frac{\phi}{\phi}>\frac{Q_{2 m-1}^{*}}{Q^{*}}=\alpha_{1}^{-1} \frac{\alpha_{3} \alpha_{5} \ldots \alpha_{2 m-1}}{\alpha_{4} \alpha_{6} \cdots \alpha_{2 m-2}}>1 ; F_{2 m-1}>F ; \\
\frac{\phi}{\phi_{2 m}}<\frac{Q_{2 m}^{*}}{Q^{*}}=\alpha_{1} \frac{\alpha_{46} \alpha_{6} \ldots \alpha_{2 m}}{\alpha_{35} \alpha_{5} \cdots \alpha_{2 m-1}}<1 ; F_{2 m}<F_{0}
\end{gathered}
$$

We see that, for $n \geqq 3$, successive approximations give $\left|Q_{n}^{*}\right|$ alternately larger ( $\mathrm{n}$ odd) and smaller ( $\mathrm{n}$ even) than $\left|Q^{*}\right|$ and that the magnitude of the fractional error increases. $\phi_{n}$ behaves 
similarly but in opposite phase: the $\phi_{n}{ }^{\prime}$ s are alternately smaller ( $n$ even) and larger ( $n$ odd) than $\phi$ with the magnitude of the fractional error increasing in the following integral

sense. In view of equation (25)

$$
2 Q_{n+1}^{*}=\int_{\theta_{0}}^{\theta_{1}} \phi_{n} d \theta=S_{n},
$$

so that the fractional error of $S_{n}$ is exactly that of $Q_{n+1}^{*}$ (and hence somewhat larger in magnitude than that of $Q_{n}^{*}$ ). It is convenient also to introduce $\Delta_{n}$ through the equation

$$
\Delta_{n}=S_{n}-2 Q_{n}^{*} \text {, i.e. } \Delta_{n}=2 Q_{n+1}^{*}-2 Q_{n}^{*} \text {. }
$$

$\Delta_{n}$ is the discrepancy in satisfaction of integral continuity for the nth approximation. Evidently $\Delta_{I}=0$ and successive approximations give $\Delta_{n}$ alternately positive ( $n$ even) and negative ( $\mathrm{n}$ odd) and increasing in magnitude. See Tables 1 and 2 and Figures 1-4.

Thus Parlange's method fails to converge, with successive approximations making oscillations of increasing magnitude about the exact solution. It is likely that $F_{n}$ converges fairly rapidly to $F$, and $\alpha_{n}$ to the value 1 , so that, in due course, the oscillations settle down to an essentially steady cycle. Our illustrative examples confirm this conjecture (Tables 1 and 2 ).

Example 1: the 'linear' soil. We follow Parlange (1971a) in taking as an illustrative example the 'linear' soil with $D=D_{*}$ = constant. Without loss of generality we take $D_{*}=1 \mathrm{~cm}^{2} \mathrm{sec}^{-1}$, 
$\theta_{0}=0, \theta_{1}=1 . \phi_{1}$ and $\phi_{2}$ may be found analytically, but the higher approximations require numerical quadratures, which we performed on the CSIRO CDC 3600, using the trapezoidal approximation and 100 steps in $\theta_{0}$ Table 1 compares various properties of the first six approximations of Parlange's method with the well-known exact solution; the oscillatory and nonconvergent behavior of $Q_{n}^{*}$ and $S_{n}$ is shown in Figure $I$; and Figure 2 compares the nonconvergent sequence of $\phi_{n}$ profiles with the exact $\phi$. Note that the root-mean-square fractional error, based on the nine $\theta$-points 0.9 to 0.1 is $11 \%$ for $\phi_{1}$, but increases to almost $17 \%$ for $\phi_{2}$. Curiously, Parlange (1971a) ignores the better fit of $\phi_{1}$ and compares $\phi_{2}$ and $\phi$ in his Table 2. Systematic small errors in many of his values of both $\phi_{2}$ and $\phi$ lead Parlange to underestimate the error of $\phi_{2}$.

[Insert Table $I$ and Figures $I$ and 2 here].

Example 2: Yolo light clay. Our second example, Yolo light clay with $\theta_{0}=0.2376, \theta_{1}=0.4950$, was used also by Parlange (2). The relevant $D(\theta)$ function is tabulated in Philip (1957a) and graphed in Philip (1969). We carried out the required numerical quadratures on the CSIRO CDC 3600, using the trapezoidal approximation and 100 steps in $\theta$. Table 2 compares various properties of the first six of Parlange's approximations with the exact solution, for which we adopt the values secured by Phllip (1957a) using the numerical technique of Philip (1955). Philip's solution involves correction of truncation errors and an analytical solution near the singularity at $\theta_{0}$, and it is apparently correct 
to four significant figures. Figure 3 depicts the oscillatory and nonconvergent behavior of $Q_{n}^{*}$ and $S_{n}$, and Figure 4 compares the nonconvergent sequence of $\phi_{n}$ profiles with $\phi$. In agreement with inequalities developed above, our numerical calculations (Table 2) give $\phi_{1}$ definitely greater than $\phi$ in $0.53 \leqq \Theta<1$ and $\phi_{2}$ definitely greater than both $\phi_{1}$ and $\phi$ throughout the range $0<\Theta<1\left(\theta_{0}<\theta<\theta_{1}\right)$.

[Insert Table 2 and Figures 3 and 4 here]

Figure $I$ of Parlange (1971a) shows $\phi_{1}, \phi_{2}$, and $\phi$ for this example, but fails to exhibit the foregoing behavior, and is incorrect. Parlange represents $\phi_{1}, \phi_{2}$, and $\phi$ for this example as indistinguishable in $0.5 \leqq \Theta \leqq 1$, and $\phi_{2}$ and $\phi$ as almost so in $0.1<\Theta<0.5$. His $\phi_{2}$ is, in fact, less than than $\phi$ in $0<\Theta<0.1$. We remark on these discrepancies, because Parlange appeals to the agreement between $\phi$ and $\phi_{2}$ in his Figure 1 as evidence of the success of his method.

\section{Other phenomena subject to concentration conditions}

The foregoing analysis reveals the unsatisfactory consequences of the absence of any constraining link between the 'continuity' step and the 'Darcy law' step in each iteration. There is no reason to expect Parlange's procedure to be any more successful for more complicated phenomena subject to concentration conditions. 
It follows immediately, for example, that the method definitely fails to converge in the limit of $t$ small for one-dimensional infiltration and two- and three-dimensional sorption subject to constant concentration conditions. Although detailed work has yet to be done on this point, it appears that the method, applied to these processes, does not converge.

Phenomena subject to flux conditions.

Parlange has not proposed a specific iterative procedure for solutions subject to flux conditions. Here also, however, his second approximation abandons integral continuity and is consequently unsatisfactory. Check calculations on one-dimensional sorption subject to constant flux condtions confirm that, as expected, the first approximation is superior to the second in this case also.

\section{THE ACCURACY OF THE FIRST APPROXIMATION}

As we have seen, the Macey-Parlange first approximation gives a better estimate of the integral properties of the solution than do Parlange's higher approximations and it satisfies integral continuity, unlike the higher approximations ${ }^{5}$. In these circumstances, the utility of Parlange's method is simply the utility of the first approximation : we therefore examine the accuracy of this approximation. For a given phenomenon, this has two major determinants: (a) the 'shape' of the diffusivity function $D(\theta) ;(b)$ the accuracy with which relation (15) represents the relevant exact $F(\theta)$ function.

\section{The 'shape' of the diffusivity function}

The exact integrals which correspond to those in the first approximation have $Q F$ in place of $Q_{1}$. Both the exact and the 
approximate integrals have the factor $D$ in the numerator of their integrands and have $\theta_{1}$ as their fixed upper limit. Now, since $F\left(\theta_{1}\right)=1$, the errors in the integrals of the first approximation will be small when $\mathrm{D}$ is strongly peaked at $\theta=\theta_{1}$. In fact, the error is demonstrably zero in the limiting case of absorption (or infiltration) in the delta-function soil, even though the correct $\mathrm{F}$ deviates strongly from relation (15) Part 1). Parlange (197la) recognized that his method is best adapted to soils with $D$ strongly peaked at $\theta_{1}$.

\section{The accuracy of relation (15)}

The diffusivity function for real soils may deviate appreciably fro delta-function at $\theta=\theta_{1}$. In such cases the accuracy of the MaceyParlange first approximation depends also on the closeness of relation (15) to the relevant exact $F(\theta)$ function. The investigation of $F(\theta)$ reported in Part 16 leads to the following conclusions from this aspect:

(a) Parlange's initially assumed $\mathrm{F}$ is more accurate for : desorption than for absorption in real soils [ cf.Figure 1 of Part 1].

(b) It is not well-fitted to one-dimensional infiltration [Figure 2 of Part 1], but is better fitted to capillary rise.

(c) It is less accurate for phenomena subject to variable concentration conditions, than for phenomena subject to constant concentration conditions [Figure 3 of Part 1].

(d) It is relatively well-fitted to two- and threedimensional sorption in the limit of large $t$.

THE RESTRICTION TO ONE SPACE COORDINATE

Parlange (197lc) specifically treats two- and three-dimensional sorption in radially symmetrical systems subject to constant concentration conditions. He asserts, however, that the method applies to sorption from cylinders and three-dimensional cavities of arbitrary shape and he claims 
that 'the great interest of the present method is that it is easily adaptable to any geometry'. This claim is, in fact, mistaken.

Parlange fails to recognize that, for two- and three-dimensional systems which are not radially symmetrical, his $g$ (our $Q^{-1}$ ) is necessarily a function, not only of $t$, but also of one $(m=2)$ or two $(m=3)$ space coordinates on the water supply surface $w$. The procedure suggested in the final section of Parlange (1971c) is therefore irrelevant and erroneous. We see that Parlange's method can yield the required ordinary differential equation in $Q(t)$ only for systems specified by one space coordinate; and this restriction holds also for cognate techniques such as those we develop in Part 3.

In order to illuminate the issues and to clear the ground for further progress, we have been obliged, in this paper, to emphasize various shortcomings of Parlange's work. This must not, however, detract from the great credit due to Parlange for his imaginative and stimulating attack on the flow equation. 


\section{References:}

Klute, A. 1952 A numerical method for solving the flow equation for water in unsaturated materials. Soil Sci.73: 105-116. Macey, R.I. 1959 A quasi-steady-state approximation method for diffusion problems: I. Concentration dependent diffusion coefficients. Bull.Math.Biophys. 21: 19-32.

Parlange, J.-Y. 197la Theory of water movement in soils: I. Onedimensional absorption. Soil Sci.111: 134-137.

Parlange, J.-Y. 1971b Theory of water movement in soils: II. One-dimensional infiltraton. Soil Sci. 111: 170-174.

Parlange, J.-Y. 197lc Theory of water movement in soils: III. Two and three dimensional absorption. Soil Sci. 112: 313-317. Parlange, J.-Y. 1972 Theory of water movement in soils: VIII. Onedimensional infiltration with constant flux at the surface. Unpublished manuscript.

Philip, J.R. 1955 Numerical solution of equations of the diffusion type with diffusivity concentration-dependent. Trans.Faraday Soc.51: 885-892.

Philip, J.R. 1957a. The theory of infiltration: 1. The infiltration equation and its solution. Soil Sci. 83: 345-357.

Philip, J.R. 1957b The theory of infiltration: 4. Sorptivity and algebraic infiltration equations. Soil Sci. 84: 278-286.

Philip, J.R. 1969 Theory of infiltration. Adv.Hydrosci. 5: 215-296. Philip, J.R. 1973 on solving the unsaturated flow-equation: 1. The flux-concentration relation. Soil Sci. : . Philip, J.R., and Knight, J. H. 1973 on solving the unsaturated flow equation: 3 . Soil Sci 


\section{Footnotes:}

1

Research School of Physical Sciences, P.O. Box 4, Canberra, A.C.T. 2600 and CSIRO Division of Environmental Mechanics.

2

Division of Environmental Mechanics, P.O. Box 821, Canberra City, A.C.T. 2601. Please direct reprint requests to this address. We are grateful to Professor Parlange for access to Parlange (1972) before its publication.

3 However Parlange is not wholly consistent in applying his method: Without warning he iterates in a different way (still abandoning integral continuity) for one problem, that of threedimensional absorption (Parlange, 1971c). The following explanation thus does not apply in detail to that one case.

4 Note that $g$ in Parlange (197la) and C in Parlange (1971b) are both equivalent to $-Q^{-1}$ in our notation; and $g$ in Parlange (1971c) corresponds to our $r_{0}{ }^{1-m_{Q}}{ }^{-1}$. Parlange does not interpret these quantities physically.

5

Each higher approximation improves the 'shape' of the moisture profiles, but increases the error of the absolute values.

6

We recall that relation (19) of Part 1 is relation (15) of the present paper. 


\section{Tables:}

TABLE 1

Comparison of Parlange's first six approximations with exact solution for sorption in the 'linear' soil

\begin{tabular}{c|cccccc|c}
$n$ & 1 & 2 & 3 & 4 & 5 & 6 & $\begin{array}{l}\text { Exact } \\
\text { values }\end{array}$ \\
\hline$\phi_{n}(0.9)$ & 0.200 & 0.201 & 0.145 & 0.223 & 0.140 & 0.225 & 0.178 \\
$\phi_{n}(0.8)$ & 0.400 & 0.405 & 0.292 & 0.450 & 0.283 & 0.454 & 0.358 \\
$\phi_{n}(0.7)$ & 0.600 & 0.619 & 0.444 & 0.685 & 0.431 & 0.691 & 0.545 \\
$\phi_{n}(0.6)$ & 0.800 & 0.847 & 0.603 & 0.933 & 0.586 & 0.941 & 0.742 \\
$\phi_{n}(0.5)$ & 1.000 & 1.099 & 0.773 & 1.200 & 0.754 & 1.210 & 0.954 \\
$\phi_{n}(0.4)$ & 1.200 & 1.386 & 0.962 & 1.499 & 0.940 & 1.510 & 1.190 \\
$\phi_{n}(0.3)$ & 1.400 & 1.735 & 1.179 & 1.849 & 1.157 & 1.860 & 1.466 \\
$\phi_{n}(0.2)$ & 1.600 & 2.197 & 1.447 & 2.291 & 1.430 & 2.300 & 1.812 \\
$\phi_{n}(0.1)$ & 1.800 & 2.944 & 1.835 & 2.954 & 1.834 & 2.955 & 2.326 \\
\hline $2 Q_{n}^{*}$ & 1.000 & 1.000 & 1.384 & 0.898 & 1.428 & 0.890 & 1.128 \\
$S_{n}$ & 1.000 & 1.384 & 0.898 & 1.428 & 0.890 & 1.432 & 1.128 \\
$A_{n}$ & 0.000 & +0.384 & -0.486 & +0.529 & -0.538 & +0.542 & 0.000 \\
\hline
\end{tabular}


TABLE 2

Comparison of Parlange's first six approximations with exact solution for absorption in Yolo light clay

\begin{tabular}{c|ccccccc}
$n$ & 1 & 2 & 3 & 4 & 5 & 6 & $\begin{array}{c}\text { Exact } \\
\text { values }\end{array}$ \\
\hline $10^{2} \phi_{n}(0.9) \dagger$ & 3.021 & 3.051 & 2.589 & 3.136 & 2.577 & 3.138 & 2.852 \\
$10^{2} \phi_{n}(0.8)$ & 3.757 & 3.846 & 3.256 & 3.946 & 3.243 & 3.949 & 3.575 \\
$10^{2} \phi_{n}(0.7)$ & 4.167 & 4.333 & 3.660 & 4.437 & 3.646 & 4.440 & 4.018 \\
$10^{2} \phi_{n}(0.6)$ & 4.481 & 4.753 & 4.002 & 4.854 & 3.988 & 4.857 & 4.395 \\
$10^{2} \phi_{n}(0.5)$ & 4.776 & 5.208 & 4.365 & 5.298 & 4.352 & 5.300 & 4.796 \\
$10^{2} \phi_{n}(0.4)$ & 5.108 & 5.823 & 4.844 & 5.886 & 4.835 & 5.886 & 5.329 \\
$10^{2} \phi_{n}(0.3)$ & 5.420 & 6.541 & 5.387 & 6.558 & 5.385 & 6.558 & 5.939 \\
$10^{2} \phi_{n}(0.2)$ & 5.602 & 7.126 & 5.815 & 7.090 & 5.820 & 7.088 & 6.415 \\
$10^{2} \phi_{n}(0.1)$ & 5.736 & 7.848 & 6.321 & 7.723 & 6.338 & 7.720 & 6.984 \\
\hline $2 \times 10^{2} Q_{n}^{*}$ & 1.1697 & 1.1697 & 1.3765 & 1.1367 & 1.3830 & 1.1358 & 1.2538 \\
$10^{2} S_{n}$ & 1.1697 & 1.3765 & 1.1367 & 1.3830 & 1.1358 & 1.3832 & 1.2538
\end{tabular}




\section{Figure Legends}

FIG. 1. Parlange's first six approximations to $2 Q^{*}$ and $S$ for Example 1, sorption in the linear soil, compared with the exact value. Crosses connected by dashed lines denote $2 Q^{*}$; open circles connected by dotted lines denote $S_{n} \circ \Delta_{n}\left(=S_{n}-2 Q_{n}^{*}\right)$ is the discrepancy in satisfaction of integral continuity for the $n$th approximation. $Q_{n}^{*}$ is the surface flux density in reduced form and $s_{n}$ the sorptivity, as given by the nth approximation. The exact result is $2 Q^{*}=S$.

FIG. 2. Parlange's first four approximations, $\phi_{1}, \phi_{2}, \phi_{3}$, $\phi_{4}$ for Example 1 , sorption in the linear soil, compared with the exact solution, $\phi . \theta$ is volumetric moisture content; $\phi(\theta)$ and $\phi_{n}(\theta)$ are moisture profiles in reduced form (exact and according to the nth approximation, respectively).

FIG. 3. As Figure 1 for Example 2, absorption in Yolo light clay.

FIG. 4. As Figure 2 for Example 2, absorption in Yolo light clay; first three approximations only. 
$$
-2-
$$

Complete Title:

ON SOLVING THE UNSATURATED FLOW EQUATION : 3 . NEW QUASI-ANALYTICAL TECHNIQUE 
Authors' Names and Affiliations:

\author{
J.R.PHILIP 1 \\ CSIRO, Australia \\ and
}

J.H. KNIGHT ${ }^{2}$

Australian National University

Received for publication...August. 15.2972.... 


\section{Abstract}

We present a new quasi-analytical technique for solving the

flow equation. It has affinities with Parlange's method, but

offers the following advantages: freedom to choose an initial

assumed flux-concentration relation, $F_{1}$, greatly improves the possible accuracy of the first approximation: the higher approximations preserve integral continuity and therefore behave more stably. The first of these advantages is of practical importance, but the second ismore basic. This paper treats only solutions subject to concentration conditions: the related technique for solutions subject to flux conditions will be developed in a later paper. The technique is studied analytically and numerically for one-dimensional sorption subject to constant concentration conditions. It is found to be convergent for a wide range of shapes of the diffusivity function. For the unfavorable case of the 'linear' soil, the mean error is $3 \%$ after two iterations and $1 \%$ after three. For absorption in Yolo light clay the corresponding figures are $0.57 \%$ and $0.07 \%$

The general iterative scheme for one-dimensional infiltration subject to constant concentration conditions is presented. Three choices of $F_{1}$ should yield useful first approximations: (A) $F_{1 A}=\lim _{t \rightarrow 0} F$ ( $t$ is time) $=F_{\text {abs }}$, the $F$ for the analogous absorption process; (B) $F_{1 B}=\lim _{t \rightarrow \infty} F=\theta$; and (C) $F_{1 C}$, an interpolation function which is exact in the limits as $t \rightarrow 0$ and $t \rightarrow \infty . F_{1 A}$ should lead to a good lower bound for the infiltration rate function $q(t), F_{1 B}$ an upper bound, and $F_{l_{C}}$ a close upper bound for all except very large $t$, and the quality of the estimates of moisture profiles should be comparable. Detailed calculations for Yolo light clay bear out these expectations: the three estimates are wholly consistent with the power series solution of Philip (1957b). The error of the approximation 
based on $F_{1 C}$ increases from $0 \%$ at $t=0$ to about $1 \%$ at $t=10^{6}$ sec. This first approximation is accurate enough to render iteration unnecessary for most purposes. Parallel calculations confirm the nonconvergence of Parlange's method when applied to infiltration.

General iterative schemes are given also for two- and threedimensional sorption subject to constant concentration conditions. 
The concept of the flux-concentration relation in flow

phenomena in unsaturated soils was introduced in Part 1 (Philip, 1972) and used in Part 2 (Knight and Philip, 1972), which treated various shortcomings of the method of solving the unsaturated flow equation due to Parlange (197la). In this Part 3 we develop a new technique which avoids these difficulties, and we use it to establish new quasi-analytical approximations to the solution of a range of problems. This paper treats only solutions subject to concentration conditions, the matter of solutions subject to flux conditions being left for a later communication. (The difference of conditions affects matters of detail, but the basic principles of the technique are unaltered).

We showed in Part 2 that Parlange's first approximation satisfies integral continuity, but that this is abandoned in his higher approximations, which make oscillations of progressively larger amplitude about the exact solution. The technique here, on the other hand, preserves integral continuity in each approximation and has the second (and lesser) advantage that it allows a better initial estimate of $F(\theta)$, the flux-concentration relation. It is clear from examples that our technique is rapidly convergent when applied to appropriate problems

though we establish convergence analytically for certain special cases only.

As developed in this paper, the technique applies to the solution of any of the specialized flow equations (1) $-(\underline{3})$ subject to initial condition (4) and boundary condition (6), which includes condition (5) as a special case:

$$
\begin{aligned}
& \frac{\partial \theta}{\partial t}=\frac{\partial}{\partial x}\left(D \frac{\partial \theta}{\partial x}\right) ; \\
& \frac{\partial \theta}{\partial t}=\frac{\partial}{\partial z}\left(D \frac{\partial \theta}{\partial z}\right)-\frac{d K}{d \theta} \frac{\partial \theta}{\partial z} ;
\end{aligned}
$$




$$
\begin{aligned}
& \frac{\partial \theta}{\partial t}=r^{1-m} \frac{\partial}{\partial r}\left[\operatorname{Dr}^{m-1} \frac{\partial \theta}{\partial r}\right] ; \\
& t=0, \theta=\theta_{0} \text { in } x>0 \text { or } z>0 \text { or } r>r_{0} ; \\
& t \geqslant 0, \theta=\theta_{1} \text { at } w_{;} \\
& t \geqslant 0, \theta=\theta_{1}(t) \text { at } w .
\end{aligned}
$$

We use the same symbolism as in Parts 1 and 2, where these equations and conditions are discussed more fully. As previously, $W$ denotes $x=0, z=0$, or $r=r_{0}$. We shall again have occasion to refer to the specialized continuity equations in integrated form

$$
v=\int_{\theta}^{\theta}(\partial x / \partial t) d \theta ; v-k_{0}=\int_{\theta}^{\theta}(\partial z / \partial t) d \theta ; m r^{m-1} v=\int_{\theta}^{\theta}\left(\partial r^{m} / \partial t\right) d \theta \cdot(\underline{z})
$$

$\mathrm{K}_{0}=\mathrm{K}\left(\theta_{0}\right)$. We have, then, for $\theta=\theta_{1}$,

$$
Q=\int_{\theta}^{\theta}(\partial x / \partial t) d \theta, Q-K_{0}=\int_{\theta}^{\theta}(\partial z / \partial t) d \theta, m_{0}^{m-1} Q=\int_{\theta}^{1}\left(\partial r^{m} / \partial t\right) d \theta \cdot(\underline{8})
$$

We also use the specialized form of Darcy's law,

$$
\mathrm{v}=-\mathrm{D} \partial \theta / \partial \mathrm{x} ; \quad \mathrm{v}=\mathrm{K}-\mathrm{D} \partial \theta / \partial \mathrm{z} ; \quad \mathrm{v}=-\mathrm{D} \partial \theta / \partial \mathrm{r} \text {. }
$$

Further, we recall the definition of $F(\theta)$ (Part 1)

$$
\theta=\frac{\theta-\theta_{0}}{\theta_{1}-\theta_{0}}, F=\frac{v}{Q} \text { or } \frac{v-K_{0}}{Q-K_{0}} \text { or }\left(\frac{r}{r_{0}}\right)^{m-1} \frac{v}{Q}
$$

and that (Part 2)

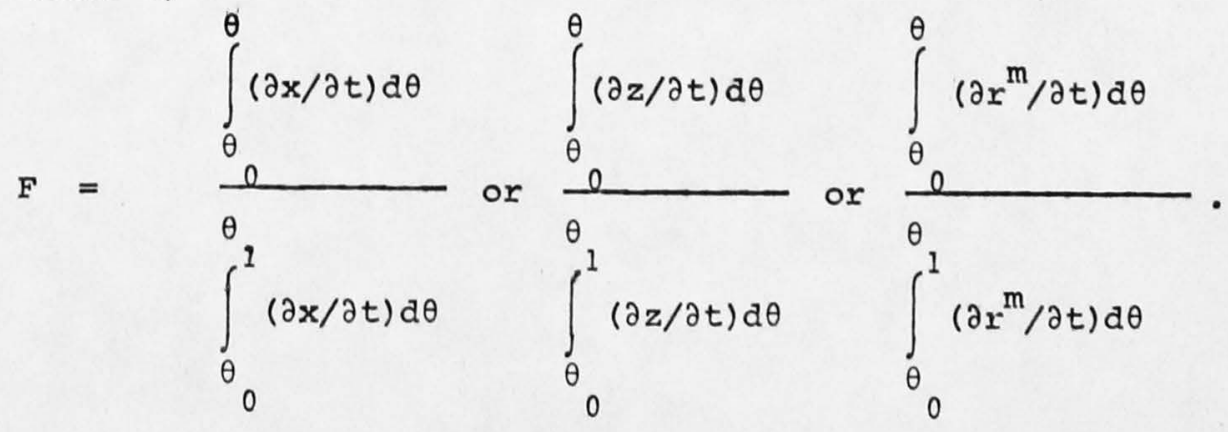




\section{GENERAL DESCRIPTION OF THE TECHNIQUE}

our technique consists of using an iterative procedure to establish the 'shape' of the solution: then, once the 'shape' is fixed, $Q$, and hence the 'magnitude' of the solution, are found through the integral continuity requirement ( 1 ).

More specifically, the characteristic of the solution which we aim to fix by iteration is the flux-concentration relation F. We start with an assumed $F$ and use an iterative procedure of integrating Darcy's law ( $\underline{9})$ and putting the resulting estimate of $\mathrm{y}(\theta, Q)$ into equation ( $\underline{8})$. [ As in Part 2 , we use $\mathrm{y}$ to denote the appropriate space coordinate, $x$ or $z$ or $r$.$] This yields a$ first order differential equation, the solution of which gives an estimate of $Q(t)$ and hence an estimate of $y(\theta, t)$. Equation (11) then provides a new estimate of $\mathrm{F}$; and so on. When we elect to terminate the calculation, we use the final estimate of $F$ in a last truncated iteration which stops at the final estimate of $y(\theta, t)$. Our first approximation differs from that of Parlange (1971a), described in Part 2, only in that we are not limited to Parlange's assumed initial F [ relation (19) of Part 1 and relation (15) of Part 2]. Knowledge of the general behavior of $\mathrm{F}$, as developed for example in Part 1, leads to the use of other $\mathrm{F}_{1}$ 's, which are often more accurate and more convenient. ${ }^{3}$

The fundamental difference between our procedure and Parlange's, however, lies in the higher approximations. We impose the constraint of integral continuity in each iteration by rewriting the appropriate equation ( $\underline{8}$ ) as a first order differential equation giving a fresh estimate of $Q(t)$ : and this is necessarily consistent with the general estimate of $\int_{\theta}^{\theta_{1}}(\partial y / \partial t) d \theta$ [or of $\int_{\theta_{0}}^{\theta}\left(\partial r^{m} / \partial t\right) d \theta$ ]. 
We proceed to apply the technique to particular problems. As we shall see, in the special cases of phenomena subject to constant concentration conditions, our procedure is simplified in the sense that there is no need to explicitly calculate estimates of $Q$ and $y$ in each iteration : we are able to go directly from one estimate of $F$ to the next.

\section{ONE-DIMENSIONAL SORPTION: CONSTANT CONCENTRATION CONDITIONS}

As in Part 2, the similarity character of the solution of equation (1) subject to conditions (ㄴ), () enables us to discuss it in terms of reduced quantities. We use the symbolism

$$
\phi=x t^{-\frac{1}{2}}, v=v t^{\frac{1}{2}}, s=\int_{\theta}^{\theta} \phi d \theta=2 Q t^{\frac{1}{2}} .
$$

Here S is, as usual, the sorptivity (Philip 1957d, 1969). In Part 2 we distinguished between $2 Q^{*}\left(=2 Q t^{\frac{3}{2}}\right)$ and $S$ because Parlange's second and higher approximations give different values for these quantities. There is no need to discriminate between $2 Q^{*}$ and $S$ here, because our preservation of integral continuity ensures their identity in all approximations.

\section{The iterative scheme}

The nth iteration begins with the nth estimate of $F, F_{n}$. For $n=1$ this constitutes the initial assumption; for $n>1, F_{n}$ is the outcome of the $(n-1)$ th iteration. Since the $n$th estimate of $V, V_{n}=\frac{1}{2} F_{n} S_{n}$, where $S_{n}$ is the nth estimate of $s$, the first of equations (9) reduces to

$$
F_{n} S_{n}=-2 D d \theta / d \phi_{n} \text {, i.e. } \phi_{n}=2 s_{n}^{-1} \int_{\theta}^{\theta} \frac{D}{F_{n}} d \theta
$$

Integration with respect to $\theta$ and use of the third of equations (12) [which embodies the first of integral continuity requirement (8)] then gives 


$$
S_{n}=\left[2 \int_{\theta}^{\theta} \frac{\left(\theta-\theta_{0}\right) D}{F_{n}} d \theta\right]^{\frac{1}{2}} \text {, }
$$

so that we may rewrite equation $(\underline{13})$ as

$$
\phi_{n}=\left[\frac{1}{2} \int_{\theta_{0}}^{\theta} \frac{\left(\theta-\theta_{0}\right) D}{F_{n}} d \theta\right]^{-\frac{1}{2}} \int_{\theta}^{\theta} \frac{D}{F_{n}} d \theta .
$$

Finally, we use equation (11) [ or equivalently equation of Part 1] to yield the basis for the next iteration, the $(n+1)$ th estimate of $F$,

$$
\int_{\theta}^{\theta}\left[\int_{\theta}^{\theta} \frac{D}{F_{n}} d \theta\right] d \theta
$$

$=$

$$
\int_{\theta}^{1} \frac{(\theta-\theta) D}{F_{n}} d \theta
$$$$
\int_{\theta}^{\theta} \frac{(\beta-\theta) D(\beta)}{F_{n}(\beta)} d \beta
$$$$
\text { or } 1 \text { - }
$$

The final expression is more useful in computations. Alternatively, equation $(16)$ is obtained by dividing equation (28) of Part 2 by equation (29) of Part 2 and replacing $n$ in the result by $(n+1) \cdot 4$

\section{Equation (16) constitutes the essential scheme for all} iterations except the final truncated one, which terminates with the . ealeulation of $s_{n}$ and $\phi_{n}$ through equations $(\underline{14})$ and $(\underline{15})$. This calculation is inessential to the previous iterations.

\section{Convergence of the iterative process}

When $F_{n}=F_{n+1}=F$, equation $(\underline{16})$ becomes

$$
F=\frac{\int_{\theta}^{\theta}\left[\int_{\theta}^{\theta} \frac{D}{F} d \theta\right] d \theta}{\int_{\theta}^{1} \frac{\left(\theta-\theta_{0}\right) D}{F} d \theta} .
$$


Equivalently, equation (17) follows directly from the exact solution, for example from equations $(\underline{21)}$ and $(\underline{30})$ of Part 2 . Differentiating equations $(\underline{16})$ and $(\underline{17)}$ with respect to $\theta$ and dividing the first result by the second gives the relation

$$
\frac{d F_{n+1}}{d F}=\frac{\int_{\theta}^{\theta} \frac{F}{F_{n}} d G(\theta) / \int_{\theta}^{1} \mathrm{dG}(\theta)}{\int_{\theta}^{1} \frac{\left(\theta-\theta_{0}\right) F}{F_{n}} d G(\theta) / \int_{\theta}^{\theta}\left(\theta-\theta_{0}\right) d G(\theta)},
$$

where $G=\int \frac{D}{F} d \theta$. We use equation $(\underline{18)}$ in the following examination of the convergence of iterative scheme $(16)$.

A complete and general study promises to be difficult and lengthy, and we limit ourselves here to the family of diffusivity functions defined by

$$
D \propto \theta^{p}-\theta^{2 p} /(1+p), p>0 .
$$

Relation (19) covers a very wide range of shapes of D. The case $p \rightarrow 0$ is appropriate to desorption $\left(\theta_{1}<\theta_{0}\right)$ in a soil with a fairly marked peak in $D$ at $\theta=\theta_{0} ; p=\frac{1}{2}(\sqrt{3}-1) \approx 0.366$ represents a virtually constant $D$; and the case $p \rightarrow \infty$ is appropriate to absorption $\left(\theta_{1}>\theta_{0}\right)$ in a 'delta-function' soil. We estimate that the D-shapes appropriate to absorption in real soil may be represented reasonably well by the range $\frac{1}{2} \sqrt{3}-1 \leqslant p \leqslant 8$ : the value $p=4$ gives a fair match to the $D$ for Yolo light clay used in absorption and infiltration examples in this series. The range $0<p<\frac{1}{2} \sqrt{3}-1$ provides a model of desorption in soils with $D$ increasing moderately with $\theta$. Without loss of generality we take $\theta_{1}=1, \theta_{0}=0$ in the remainder of this subsection, so that equation (19) becomes

$$
D \propto \theta^{p}-\theta^{2 p} /(1+p) \text {. }
$$


The solution of equation (I) subject to conditions

(4), () with D given by equation $(\underline{20)}$ is well-known [ entry No. 1 of Table 1 of Philip (1960)] and it follows at once that for this solution

$$
F=p^{-1}\left[(p+1) \theta-\theta^{p+1}\right] \text { and } \frac{D}{F} \propto \theta^{p-1} .
$$

Equation (18) then becomes

$$
\frac{d F_{n+1}}{d F}=\frac{p \int_{\theta}^{1} \theta^{p-1} \frac{F}{F_{n}} d \theta}{(p+1)\left(1-\theta^{p}\right) \int_{0}^{1} \theta^{p} \frac{F}{F_{n}} d \theta} .
$$

If we now suppose that

$$
\frac{F}{F_{n}}=1+\alpha(1-\theta), \alpha>0,
$$

equation $(\underline{22})$ reduces to

$$
\frac{d F_{n+1}}{d F}=1+\frac{\alpha}{(p+1)(p+2+\alpha)}\left[\frac{1-(m+1)^{2} \theta^{p}+p(p+2) \theta^{p+1}}{1-\theta^{p}}\right]
$$

Since $d F / d \theta=p^{-1}(p+1) /\left(1-\theta^{p}\right)$, integration yields

$$
\frac{F_{n+1}}{F}=1+\frac{\alpha H}{(p+1)(p+2+\alpha)} \text {, }
$$

where

$$
H=1-\frac{\frac{p(p+2)}{p+1} \theta^{p}-p \theta^{p+1}}{1-\theta^{p} /(p+1)} \text {. }
$$

We note that $H$ varies from 1 to $O$ as $\theta$ varies from 0 to 1 , that

$$
0 \leqslant \theta \leqslant 1,1 \geqslant \mathrm{H} \geqslant 0,
$$

and that

$$
0 \leqslant \theta \leqslant 1, H \leqslant 1-\left(1+\frac{\theta^{p}}{p+1}\right)\left(\frac{p(p+2)}{p+1} \theta^{p}-p \theta^{p+1}\right) .
$$

Integration establishes that the mean value of $\mathrm{H}$ over $0 \leqslant \theta \leqslant 1$, $\overline{\mathrm{H}}$, is such that 


$$
0 \leqslant \bar{H} \leqslant \frac{4 p^{4}+10 p^{3}+9 p^{2}+6 p+4}{2(p+1)^{2}(p+2)(2 p+1)} .
$$

It therefore follows that

$$
1 \leqslant \frac{\bar{F} n+1}{F} \leqslant 1+\frac{\left(4 p^{4}+10 p^{3}+9 p^{2}+6 p+4\right) \alpha}{2(p+1)^{3}(p+2)(p+2+\alpha)(2 p+1)},
$$

where the overbar again denotes the mean over $0 \leqslant \theta \leqslant 1$. Note that changing the sign of $\alpha$ merely reverses the inequalities in (25) .

We see that each iteration changes the sign of the mean fractional error and decreases it to fraction $n$ of its former magnitude, where

$$
0 \leqslant n \leqslant \frac{4 p^{4}+10 p^{3}+9 p^{2}+6 p+4}{(p+1)^{3}(p+2)(p+2+\alpha)(2 p+1)} .
$$

\section{[ Insert Table I here]}

Table 1 gives values of

$$
\eta_{\star}=\frac{4 p^{4}+10 p^{3}+9 p^{2}+6 p+4}{(p+1)^{3}(p+2)^{2}(2 p+1)},
$$

the upper bound on $\eta$ in the limit as $\alpha \rightarrow 0$. It indicates that the iterative scheme $(\underline{16})$ converges for all $\mathrm{m}>0,5$ and that it is very rapidly convergent for the m-range appropriate to absorption in real soils. This rapid convergence is a consequence of the strong weighting of the integrals in equation (16) towards values of $\theta_{1}$ when $D(\theta)$ has a marked peak there (cf. Part 2).

We have the following supplementary results for the special case $p=1$ :

(a) With $F / F_{n}$ given by equation $(\underline{23})$,

$$
I \leqslant \frac{F_{n}}{F} \leqslant 1+\frac{\alpha}{6+2 \alpha}(1-\theta)
$$

and 


$$
\left(\overline{\frac{F+1}{F}}\right)=1+\frac{(2 \log 2-1) \alpha}{6+2 \alpha} \text {. }
$$

Here also the inequalities are reversed for $\alpha<0$.

The exact value of $\lim n$ is then 0.129 , significantly $\alpha \rightarrow 0$

less than $n_{*}(=0.153)$.

(b)

For

$$
F / F_{n}=1+\alpha(1-\theta)^{2}=1+\alpha(1-F),
$$

we obtain the exact result

$$
\frac{F n+1}{F}=1+\frac{\alpha}{6+2 \alpha}(1-F)=1+\frac{\alpha}{6+2 \alpha}(1-\theta)^{2} \text {. }
$$

\section{Illustrative examples}

Example 1: the 'linear' soil. Our first illustration is the 'linear' soil with $D=D_{*}=$ constant. Without loss of generality we take $D_{*}=1 \mathrm{~cm}^{2} \mathrm{sec}^{-1}, \theta_{0}=0, \theta_{1}=1$. We adopt $\mathrm{F}_{1}=\theta_{1} \phi_{1}$ and $\mathrm{F}_{2}$ may then be found analytically, but the further calculations require numerical quadratures, which we performed on the CSIRO CDC 3600 , using the trapezoidal approximation with 90 steps in $1>\theta>0.1$ and 100 steps in $0.1>\theta>0.6$ As we remark above, our iterative procedure goes from one estimate of $\mathrm{F}$ to the next, and only the final estimate of $\phi$ needs to be computed. For illustrative purposes, however, we have here calculated each successive $\phi_{\mathrm{n}}$. Table 2 compares the first four approximations with the well-known exact solution, and Figure 1 shows the rapid convergence of $\phi_{n}$ to the exact $\phi$. Although our technique is not especially suited to the 'linear' soil, the mean error (i.e. the error in S) is only $3 \%$ for two iterations, $1 \%$ for three, and $0.3 \%$ for four. These results are in striking contrast with the nonconvergence of Parlange's method applied to the same problem (Figure 1 and Table 1 of Part 2). 
Example 2: Yolo light clay. The second example is for Yolo light clay with $\theta_{0}=0.2376, \theta_{1}=0.4950$. The $D(\theta)$ function is tabulated in Philip (1957b) and graphed in Philip (1969). Here also we take $F_{1}=\theta$. Numerical quadratures were carried out as for Example 1. Table 3 compares the first four approximations with the adopted exact solution (Philip, 1957b) found by the numerical method of Philip (1955), and Figure 2 shows the rapid convergence of $\phi_{n}$ to the exact $\phi$. The mean error is $5.6 \%$ for the first iteration, $0.57 \%$ for the second, $0.07 \%$ for the third and $0.03 \%$ for the fourth (which is influenced by rounding errors). Evidently two iterations suffice in this case for most purposes. This should be compared with the nonconvergence of Parlange's method applied to the same problem (Figure 3 and Table 2 of Part 2). 
It will be noted that, for each example, the adoption of $F_{1}=\theta$ yields values of $\phi_{1}$ and $S_{1}$ which are too large. On the other hand, the assumption $\mathrm{F}_{1}=1$ [ as used by Parlange (1971a)] gives $\phi_{1}$ and $S_{1}$ too small. Fractional errors in $S_{1}$ are about the same for each assumption, but $\mathrm{F}_{1}=\theta$ leads to subsequent more rapid convergence, apparently because it gives the 'shape' of $\phi_{1}$ closer to that of $\phi$.

Finally, we observe that, for Example 1, errors are reduced by each iteration to about one-third their former magnitude; and, for Example 2, to about one-ninth. These very advantageous rates of convergence are in order-of-magnitude agreement with the estimates of Table 1.

\section{ONE-DIMENSIONAL INFILTRATION: CONSTANT CONCENTRATION CONDITIONS}

\section{The general iterative scheme}

We turn now to solution of equation (2) subject to conditions $(\underline{4}),(\underline{5})$. In this case integration of equation (9) (with $z_{n}$ for $z$ ) yields

$$
z_{n}(\theta, q)=\int_{\theta}^{\theta} \frac{\Delta d \theta}{q F_{n}-k},
$$

where we simplify the symbolism by writing $q$ for $Q-\mathrm{K}_{0}$ and $\mathrm{k}$ for $\mathrm{K}-\mathrm{K}_{0}$. Combining this with equation (11) (with $\mathrm{z}_{\mathrm{n}}$ for $\mathrm{z}$ ) yields the iterative procedure for $F$

$$
\begin{aligned}
& \int_{\theta}^{\theta}\left[\int_{\theta}^{\theta} \frac{D \partial\left(q F_{n}\right) / \partial q}{\left(q F_{n}-k\right)^{2}} d \theta\right] d \theta \\
& \int_{\theta}^{\theta} \frac{\left(\theta-\theta_{0}\right) D \partial\left(q F_{n}\right) / \partial q}{\left(q F_{n}-k\right)^{2}} d \theta
\end{aligned} .
$$

The differential equation in $q_{n}(t)$, the $n$th estimate of $q(t)$, obtained by putting equation (26) in equation ( 1 ) (with $z_{n}$ for $z$ ), is 


$$
\frac{d t}{d q_{n}}=-\int_{\theta}^{\theta} \frac{\left(\theta-\theta_{0}\right) D \partial\left(q_{n} F_{n}\right) / \partial q_{n}}{q_{n}\left(q_{n} F_{n}-k\right)^{2}} d \theta,
$$

which integrates to

$$
t=\int_{\theta}^{1}\left(\theta-\theta_{0}\right) D I\left[\frac{d(q F n)}{q\left(q F_{n}-k\right)^{2}}\right] d \theta .
$$

The iterative scheme is evidently complicated, and we therefore seek to optimize $\mathrm{F}_{1}$.

Case of $\mathrm{F}_{1}$ independent of $\mathrm{q}$

Firstly we note that equations $(\underline{27)}$ and $(\underline{28)}$ are simplified in the first approximation when $F_{1}$ is taken independent of $q$. They become

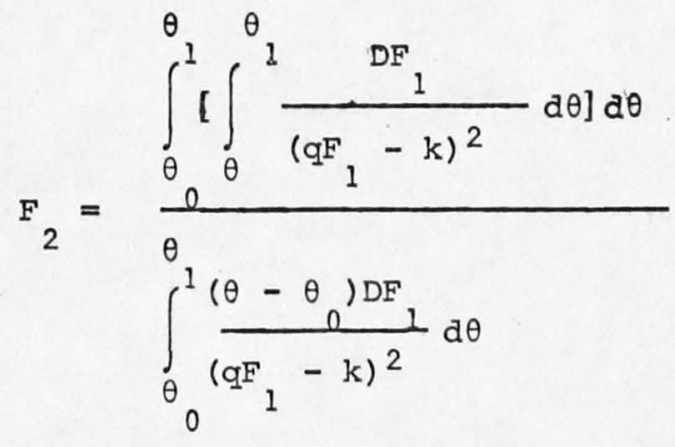

and

$$
t=\int_{\theta}^{\theta} \frac{\left(\theta-\theta_{0}\right) D F}{k^{2}}\left[\frac{k}{q_{1} F_{1}-k}-\ln \frac{q_{1} F_{1}}{q_{1} F_{1}-k}\right] d \theta .
$$

We notice that, in the special case $F_{1}=1$, equation $(\underline{30})$ is equivalent to equation (12) of Parlange (1971b).

The problem as integral equation: optimal $\mathrm{F}$

We may rewrite the infiltration problem as the integral

equation 


$$
F(\theta, a)=\frac{\int_{\theta}^{\theta}\left[\int_{\theta}^{1} \frac{D \partial(F / a) / \partial a}{(F-a k)^{2}} d \theta\right] d \theta}{\int_{\theta}^{1} \frac{\left(\theta-\theta_{0}\right) D \partial(F / a) / \partial a}{(F-a k)^{2}} d \theta} \text {, }
$$

which we obtain from equation (27) by putting $\mathrm{F}_{n+1}=\mathrm{F}_{\mathrm{n}}=\mathrm{F}$ and writing a for $\mathrm{q}^{-1}$.

We may expand the integrals in equation (31) as power series in a: the radius of convergence is the minimum value of $\mathrm{Fk}^{-1}$, namely $\mathrm{k}_{1}^{-1}, \mathrm{k}_{1}=\mathrm{K}\left(\theta_{1}\right)-\mathrm{K}_{0}$. The solution of $(\underline{31})$ is thus expressible as

$$
F(\theta, a)=\sum_{0}^{\infty} f_{i}(\theta) a^{i},
$$

where each $f_{i}(\theta)$ is the solution of an ordinary second-order differential equation obtained by equating coefficients. For $i=0$, the equation is nonlinear, but for $i>0$ it is linear. Series (32) converges in the whole interval $0 \leqslant a<k_{l}^{-1}$ (corresponding to $0 \leqslant t<\infty$ ). 8 Series (32) suggests use of an $\mathrm{F}_{1}$ with linear dependence on $a$. The resulting $F_{1}$ which satisfies the known results (Part 1 ) for $F(\theta ; a)$,

$$
F(\theta, 0)=F_{a b s}(\theta), F\left(\theta, k_{1}^{-1}\right)=\theta,
$$

(where $F_{\text {abs }}$ denotes $F$ for the analogous absorption process) is then

$$
F_{1}(\theta, a)=F_{a b s}(\theta)+f(\theta) a,
$$

where

$$
f(\theta)=k_{1}\left[\theta-F_{a b s}(\theta)\right] .
$$

Equation $(\underline{33})$ is exact at $t=0$ and as $t \rightarrow \infty$ and, in addition, yields simple expressions for $F_{2}$ and for $t\left(q_{1}\right)$ : these consist of equations $\left(\underline{29)}\right.$ and $\left(\underline{30)}\right.$ with $\mathrm{F}_{\text {abs }}$ in place of $\mathrm{F}_{1}$ and with 


$$
k^{\prime}=k+k_{1}\left[F_{a b s}(\theta)-\theta\right]
$$

in place of $\mathrm{k}$.

\section{Illustrative Example: Yolo light clay}

our example is the infiltration process in yolo light clay analogous to the absorption process of Example 2 of the preceding section. We have used our technique to compute $t\left(q_{1}\right)$ and $z_{1}(\theta, t)$ for the following $F_{1}^{\prime} S:$ (A) $F_{1}=F_{1 A}=F_{a b s}$ (B) $F_{1}=F_{1 B}=\theta$, and $(C) F_{1}=F_{1 C}$ ' the interpolated $F_{1}$ given by equation (33). We distinguish quantities calculated from the different $F_{1}$ 's by the additional suffices $A, B, C$. We have checked the accuracy of $F_{1 C}$ by comparing it with ${ }_{2 C}$, and we compare the various results with those of Philip $(1957 b, c)$. We have also computed, for comparison, $t\left(q_{1}\right), t\left(q_{3}\right), z_{1}(\theta, t)$, and $z_{2}(\theta, t)$ as given by the method of parlange $(1971 b)$ : these are identified by the suffix P. Numerical

quadratures were performed on the CSIRO CDC 3600, using the trapezoidal approximation with 100 steps in each of $1>\theta>0.9$ and $0.1>\theta>0$, and 80 steps in $0.9>\theta>0.1$. 6 Table 4 compares the various estimates of $q(t)$ and Figures 3-5 compare estimates of $z(\theta, t)$.

\section{[ Insert Table 4 and Figures 3-5 here.]}

We offer the following comments:

(i) Solution (A). Since $F=F_{1 \mathrm{~A}}$ at $t=0$ and $F \leqslant F_{1 \mathrm{~A}}$ at $t>0$, we expect $q_{1 A}(t)$ to provide a lower bound on $q(t)$. Solution (A) is a good estimate in the sense that

$$
\lim _{t \rightarrow 0} \frac{z}{z}=1, \lim _{t \rightarrow 0} \frac{q_{1}}{q}=1 \text { and } \lim _{t \rightarrow \infty}\left(q_{1}-q\right)=0 \text {. }
$$

(ii) Solution (B). Since $\lim _{t \rightarrow \infty} F=F_{1 B}$ and $F \geqslant F_{1 B}$ for $0 \leqslant t<\infty, q_{1 B}(t)$ should provide an upper bound on $q(t)$. Solution (B) will be less accurate than $(A)$ at small $t$, but it should be more accurate at large $t$ where $F \rightarrow F_{I_{B}}$. 
(iii) Solution (C). $\quad F=F_{1 C}$ both at $t=0$ and at $t=\infty$. Calculations of $\mathrm{F}_{2 \mathrm{C}}$ indicate that $\mathrm{F}_{1 \mathrm{C}}$ underestimates $\mathrm{F}$ at intermediate times by at most a few percent. We have proved that, except at very large $t, q_{1 A} \leqslant q_{1 C}<q_{l_{B}}$. Eventually, however, $q_{1 C}>q_{1 B}$. When $q_{1 C}<q_{1 B}$, we expect it to provide a close upper bound on $q(t)$ and that $z_{1 C}$ will provide an excellent estimate of $z$. These various expectations are borne out by our calculated example. The four-term series solution of Philip (1957b) is essentially exact for $t \leqslant 10^{5}$ sec: the errors increase approximately as $t^{2}$ and are estimated conservatively (Philip 1957a,c) at $0.6 \%$ in $q$ at $t=5 \times 10^{5} \mathrm{sec}$ and at $0.5 \%$ in $\mathrm{z}$ at $t=10^{6} \mathrm{sec}$. Accordingly the solution of Philip (1957b) provides a basis of comparison for $q(t)$ in $t \leqslant 5 \times 10^{5} \mathrm{sec}$ and for $z(\theta, t)$ in $t \leqslant 10^{6}$ sec.

As Table 4 indicates, $q_{1 \mathrm{~A}}$ and $\mathrm{q}_{1 \mathrm{C}}$ agree with the exact result for $t \leqslant 10^{4} \mathrm{sec}$, and $q_{1 B}$ is $4.4 \%$ too large. For $t=10^{5} \mathrm{sec}$, $q_{1 \mathrm{~A}}$ is $1 \%$ too large and $\mathrm{q}_{1 \mathrm{~B}} 2.8 \%$ too large. For $t=10^{6} \mathrm{sec}$, $q_{1 B}$ and $q_{1 C}$ have become identical and about $2 \%$ greater than $q_{1 A}$. The exact value for $t=10^{6} \mathrm{sec}$ is uncertain, but it is probable that $q_{1 \mathrm{~A}}$ is here too small by about $1 \%$ and $q_{1 B}$ and $q_{1 C}$ too large by about $1 \%$. The error of $q_{1 C}$ thus increases from zero at small $t$ to about +18 for $t=10^{6} \mathrm{sec}$.

Figures 3-5 show the corresponding properties of the estimates of $z(\theta, t) \cdot z_{1 \mathrm{~A}}$ and $z_{1 \mathrm{C}}$ are essentially exact for $t=10^{4} \mathrm{sec}$, the error of $\mathrm{z}_{1 \mathrm{~B}}$ varying from $-2 \%$ near $\theta=\theta_{1}$ to $+11 \%$ near $\theta=\theta_{0}$. For $t=10^{5} \mathrm{sec}$, the error of $z_{1 \mathrm{~A}}$ varies from $+1.5 \%$ near $\theta_{1}$ to $-2 \%$ near $\theta_{0}$; that of $z_{1 C}$ ranges from $0 \%$ to $1.5 \%$; and that of $z_{1 B}$ from $-1 \%$ to $+8 \%$. For $t=10^{6}$, the errors based on Philip (1957b) are as follows: $z_{1 \mathrm{~A}}+1.7 \%$ to $-3.6 \% ; \mathrm{z}_{1 \mathrm{C}} 0 \%$ to $1.7 \% ; \mathrm{z}_{1 \mathrm{~B}}+0.8 \%$ to $+3.2 \%$. We thus estimate that the mean error in $\mathrm{z}_{1 \mathrm{C}}$ increases 
from zero at small to less than $1 \%$ for $t=10^{6} \mathrm{sec}$. Note

that, as well as giving excellent results in the integral sense, z also gives the best estimate of the shape of $z$. At large $t z_{l_{A}}$ tends to be relatively too large near $\theta_{1}$ and too small near $\theta_{0}$. Conversely, $z_{1 B}$ tends to be relatively too small near $\theta_{1}$ and too large near $\theta_{0}$, especially at small $t$.

It is evident that all three of our first approximations are useful: $z_{1 \mathrm{~A}}$ is significantly better than $\mathrm{z}_{1 \mathrm{~B}}$, except possibly for $t \geqslant 10^{6} \mathrm{sec}$; and $\mathrm{z}_{1 \mathrm{C}}$ is accurate enough to render iteration unnecessary for most purposes.

(iv) Parlange solution. We expect that, as for absorption (Part 2), the Parlange method will yield $q_{1 \mathrm{p}}$ too small and $\mathrm{z}_{1 \mathrm{p}}$ too large near $\theta_{1}$ and too small near $\theta_{0^{\circ}}$ The second and higher approximations, which abandon integral continuity, should fail to converge, oscillating with increasing amplitude about the exact solution. Specifically, $z_{2 p}$ and $q_{3 p}$ will be too large. Our calculations confirm these expectations (Table 4 and Figures 3-5). In the limit of small $t$, the errors of the method are precisely those for absorption (Part 2): our calculations indicate that the pattern of fractional error persists for all $t>0$ at roughly the same magnitude. The claim by. Parlange (1971b) that the method is efficaceous for infiltration is not sustained: we do not find good agreement with Philip (1957b). Parlange's first approximation, $z_{1 \mathrm{p}}$, is significantly worse than our $z_{1 \mathbb{A}}:$ the mean error in $z_{1 \mathrm{P}}$ varies from $-6.7 \%$ at small $t$ to about $-5 \%$ at $t=10^{6} \mathrm{sec}$; and his second approximation, ${ }_{2} p^{\prime}$ is significantly worse than our $z_{1 B^{\prime}}$ the mean error in $z_{2 P}$ increasing from $+9.8 \%$ at small to about $+13 \%$ at $t=10^{6} \mathrm{sec}$. The increasing error of $z_{2 P}$ as $t$ increases suggests that, for infiltration, Parlange's method may not be simply nonconvergent in an ultimately steady oscillatory sense (as it is for absorption), but may diverge without limit. In any case, Parlange's higher approximations of odd 
$-37=$

progressively worse than $z_{2 P^{\circ}}$

TWO- AND THREE-DIMENSIONAL SORPTION: CONSTANT CONCENTRATION CONDITIONS Finally, we outline the application of our technique to solution of equation ( $\underline{3}$ ) subject to conditions (4), ( $\underline{5})$.

Two-dimensional sorption

The iterative scheme. For $m=2$, the iterative scheme consists of

$$
\begin{aligned}
& r_{n}(\theta, Q)=r_{0} \exp \left[\frac{1}{Q r_{0}} \int_{\theta}^{\theta_{n}} \frac{D d \theta}{F_{n}}\right] ; \\
& \int_{\theta}^{\theta}\left\{\int_{\theta}^{\theta} \frac{D}{F_{n}}\left(1+\frac{\partial \log F_{n}}{\partial \log Q}\right) d \theta \cdot \exp \left[\frac{2}{Q r} \int_{\theta}^{\theta_{1}} \frac{D d \theta}{F_{n}}\right]\right\} d \theta \\
& F_{n+1}(\theta, 2)=\frac{\theta}{\theta} \theta \text {; } \\
& \int_{\theta}^{1}\left\{\int_{\theta}^{1} \frac{D}{F_{n}}\left(1+\frac{\partial \log F_{n}}{\partial \log Q}\right) d \theta \cdot \exp \left[\frac{2}{Q r} \int_{0}^{1} \frac{D d \theta}{F_{n}}\right]\right\} d \theta \\
& t=\int_{Q_{n}}^{\infty} Q^{-3}\left[\int_{\theta_{0}}^{\theta}\left\{\int_{\theta}^{\theta} \frac{D}{F_{n}}\left(1+\frac{\partial \log F}{\partial \log Q}\right) d \theta \cdot \exp \left[\frac{2}{Q r} \int_{\theta}^{\theta} \frac{D d \theta}{F_{n}}\right]\right\} d \theta\right] d Q .
\end{aligned}
$$

only equation ( $(35)$ is needed until the last step, when equations $(\underline{34})$ and (36) yield the final estimates of $Q(t)$ and $x(\theta, t)$.

Simplifications. For $F_{1}$ taken independent of $Q$, equations $(\underline{35})$ and $(36)$ become

$$
F_{2}=\frac{\int_{\theta}^{\theta} A_{1} \exp \frac{2 A}{Q r_{0}} d \theta}{\int_{\theta_{1}}^{1} A_{1} \exp \frac{2 A}{Q x_{0}} d \theta}
$$

and

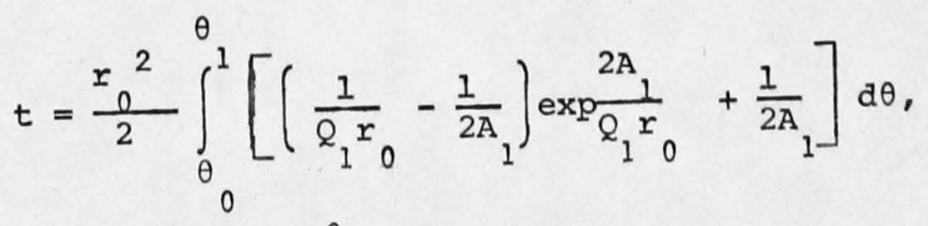

with

$$
A_{1}(\theta)=\int_{1}^{\theta_{1}} \frac{D d \theta}{F_{1}} \text {. }
$$


for the special case $F_{1}=1$, equation (37) is equivalent to equation (7) of Parlange (1971c). The search for an interpolation function $F_{1}(\theta, Q)$ which makes equation $(\underline{36})$ integrable with respect to $Q$ romalng a task for the future.

\section{Three-dimensional sorption}

The 1terattve acheme. For in 3 wo have:

$$
\begin{aligned}
& r_{n}(\theta, Q)=r_{0}\left[1-\frac{1}{Q r} \int_{0}^{1} \frac{D d \theta}{F_{n}}\right]^{-1} \text {, } \\
& \int_{\theta}^{\theta}\left\{\int_{\theta}^{1} \frac{D}{F_{n}}\left(1+\frac{\partial \log F_{n}}{\partial \log Q}\right) d \theta \cdot\left[1-\frac{1}{\partial r_{0}} \int_{\theta}^{1} \frac{D d \theta}{F_{n}}\right]^{-4}\right\} d \theta \\
& F_{n+l}(\theta, Q)=
\end{aligned}
$$

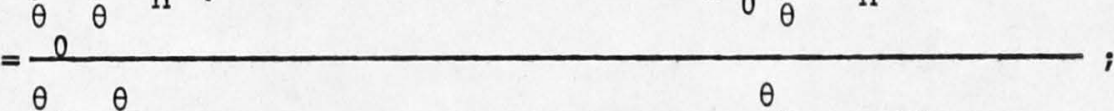

$$
\begin{aligned}
& \int_{\theta}^{1}\left\{\int_{\theta}^{1} \frac{D}{F_{n}}\left(1+\frac{\partial \log F_{n}}{\partial \log Q}\right) d \theta \cdot\left[1-\frac{1}{\partial r} \int_{0}^{1} \frac{D d \theta}{F_{n}}\right]^{-4}\right) d \theta \\
& t=\int_{Q_{n}}^{\infty} Q^{-3}\left[\int_{\theta}^{I_{0}}\left\{\int_{\theta}^{\lambda} \frac{D}{F_{n}}\left(1+\frac{\partial \log F_{n}}{\partial \log Q}\right) d \theta\left[I-\frac{1}{Q r} \int_{0}^{1} \frac{D d \theta}{F_{n}}\right]^{-4}\right\} d \theta\right] d Q .
\end{aligned}
$$

Simplifications. With $F_{1}$ independent of 2 , equations (39) and (40) become

$$
F_{2}=\frac{\int_{\theta}^{\theta} A_{1}\left[1-\frac{A_{1}}{Q r}\right]_{0}^{-4} d \theta}{\int_{\theta}^{1} \cdot A_{1}\left[1-\frac{1}{Q r}\right]_{0}^{-4} d \theta}
$$


and

$$
t=\frac{r_{0}^{2}}{6} \int_{\theta}^{\theta} A_{1}^{-1}\left[1+2\left(1-\frac{A_{1}}{Q_{1} r_{0}}\right)^{-3}-3\left(1-\frac{A_{1}}{Q_{1} r_{0}}\right)^{-2}\right] d \theta \cdot(\underline{41})
$$

For the special case $F_{1}=1$, equation (41) is equivalent to equation (20) of Parlange (1971C).

$$
\text { For this process higher approximations may involve }
$$

spurious singularities in the open interval $\theta_{0}>\theta<\theta_{1}$ and the best possibility of optimizing the solution may lie in optimizing F. 


\section{References}

Knight, J.H., and Philip, J.R. 1973. On solving the unsaturated

flow equation: 2. Critique of Parlange's method. Soil Sci. : . Parlange, J.-Y. 197la Theory of water movement in soils: I.

One-dimensional absorption. Soil Sci. 111: 134-137.

Parlange, J.-Y. 1971b Theory of water movement in soils: II. One-

dimensional infiltration. Soil Sci. 111: 170-174.

Parlange, J.-Y. 1971C Theory of water movement in soils: III. Two and three dimensional absorption. Soil sci. 112: 313-317.

Philip, J.R. 1955 Numerical solution of equations of the diffusion type with diffusivity concentration-dependent. Trans. Faraday Soc. 51: 885-892.

Philip, J.R. 1957a. Numerical solution of equations of the diffusion type with diffusivity concentration-dependent. II. Australian J. Physics 10: 29-42.

Philip, J.R. 1957b. The theory of infiltration: 1. The infiltration equation and its solution. Soil Sci. 83: 345-357.

Philip, J.R. 1957c. The theory of infiltration: 2. The profile at infinity. Soil Sci. 83: 435-448.

Philip, J.R. 1957d. The theory of infiltration: 4. Sorptivity and algebraic infiltration equations. Soil Sci. 84: 278-286.

Philip, J.R. 1960. General method of exact solution of the concentration-dependent diffusion equation. Australian J. Physics 13: 1-12.

Philip, J.R. 1966. Absorption and infiltration in two- and threedimensional systems. Unesco Symposium on Water in the Unsaturated Zone, Wageningen. 1:503-516.

Philip, J.R. 1969 Theory of infiltration. Adv. Hydrosci. 5:215-296. Philip, J.R. 1973 On solving the unsaturated flow equation: 1 .

The flux-concentration relation. Soil sci. 
$t=\frac{r_{0}^{2}\left(\theta_{1}-\theta_{0}\right)}{2}\left[\left[\frac{1}{2 r_{0}}-\frac{\theta_{1}-\theta_{0}}{s^{2}}\right] \exp \frac{s^{2}}{Q r_{0}\left(\theta_{1}-\theta_{0}\right)}+\frac{\theta_{1}-\theta_{0}}{s^{2}}\right]$,

$t=\frac{r_{0}^{2}\left(\theta_{1}-\theta_{0}\right)^{2}}{3 s^{2}}\left[1+2\left(1-\frac{s^{2}}{2 Q r_{0}\left(\theta_{1}-\theta_{0}\right)}\right)^{-3}-3\left(1-\frac{s^{2}}{2 Q r_{0}\left(\theta_{1}-\theta_{0}\right)}\right)^{-2}\right]$.

These, with changes of symbolism, areprecisely the delta-function approximations (equations (188), (178), and (181) of Philip (1969)). Compare the present equations $(\underline{30}),(\underline{37})$, and (41) . Note the connexion of this series (convergent for all finite $t$ ) with the series for $z$ in powers of $t^{\frac{1}{2}}$ (radius of convergence unknown) due to Philip (1957a, b). 


\section{Footnotes:}

1

Division of Environmental Mechanics, P.0. Box 821,

Canberra City, A.C.T., 2601. Please direct reprint requests to this address.

Research School of Physical Sciences, P.O. Box 4, Canberra,

A.C.T. 2600 and CSIRO Division of Environmental Mechanics. The paper reports, in part, studies carried out by this author in connexion with candidature for the degree of $\mathrm{Ph} . \mathrm{D}$. in the A.N.U.

We shall see that, in fact, careful estimation of $F_{1}$ may preclude the need for iteration.

4 Note that Parlange's method implicitly includes iterative scheme (16): it fails because it omits the constraint of integral continuity. Provided $|\alpha|$ is small enough in the case $\alpha<0$. The computations required in practice would be handled readily by desk calculator. We used high-speed computing because our exploratory studies demanded great accuracy and continued over many iterations. Analytical treatment of singularities, along lines similar to those of Philip (1955), may be preferred (cf. Philip, 1969, p.234) to the use here of smaller steps in $\theta$.

Note that putting the delta-function diffusivity (Part 1)

$$
\mathrm{D}=\frac{1}{2} \mathrm{~S}^{2}\left(\theta_{1}-\theta_{0}\right)^{-1} \delta\left(\theta_{1}-\theta\right)
$$

in equations $(\underline{28}),(\underline{36})$ and $(\underline{40)}$ immediately yields (for all

$n \geqslant 1)$ the respective equations

$$
t=\frac{s^{2}}{2 k^{2}}\left[\frac{k_{1}}{q-k_{1}}-\ln \frac{q}{q-k_{1}}\right]
$$


TABLE 4

Infiltration in Yolo light clay. Comparison of estimates of infiltration rate, $Q$.

\begin{tabular}{|c|c|c|c|}
\hline$t(\sec )$ & $10^{4}$ & $10^{5}$ & $10^{6}$ \\
\hline $10^{6} \mathrm{Q}$ from Philip (1957b) & 67.57 & 25.18 & 13.22 \\
\hline $10^{6} \mathrm{Q}_{1 \mathrm{~A}}$ & 67.24 & 24.92 & 13.26 \\
\hline $10^{6} \mathrm{Q}_{1 \mathrm{~B}}$ & 70.67 & 25.93 & 13.46 \\
\hline $10^{6} Q_{1 \mathrm{C}}$ & 67.94 & 25.48 & 13.46 \\
\hline $10^{6} \mathrm{Q}_{1 \mathrm{P}}$ & 63.44 & 23.98 & 13.17 \\
\hline $10^{6} Q_{3 P}$ & 74.53 & 27.78 & 15.43 \\
\hline
\end{tabular}

Notes :

Uhit of $Q$ is $\mathrm{cm} \mathrm{sec}{ }^{-1}$. Q from Philip (1957b)

is essentially exact for $t=10^{4}$ and $10^{5} \mathrm{sec}$, but of uncertain accuracy for $t=10^{6} \mathrm{sec} . Q_{1 \mathrm{~A}}$, our first approximation based on $\mathrm{F}_{1 \mathrm{~A}}$

(F for absorption). $Q_{1 B^{\prime}}$ our first approximation based on $F_{1 B}(=\theta)$. $Q_{1 C}$ ' our first approximation, based on $F_{1 C}$ (interpolated $F_{1}$ given by equation $(\underline{33}))$. $Q_{1 P^{\prime}} Q_{3 P^{\prime}}$ first and third approximations by the method of Parlange (1971b). 
TABLE 3

\section{Comparison of the first four approximations with exact solution}

for sorption in Yolo light clay, taking $F,=\theta$.

\begin{tabular}{|c|c|c|c|c|c|}
\hline $\mathrm{n}$ & 1 & 2 & 3 & 4 & $\begin{array}{l}\text { Exact } \\
\text { values }\end{array}$ \\
\hline$F_{n}(0.9) \dagger$ & 0.9 & 0.966 & 0.963 & 0.963 & 0.964 \\
\hline$F_{n}(0.8)$ & 0.8 & 0.904 & 0.895 & 0.897 & 0.896 \\
\hline$F_{n}(0.7)$ & 0.7 & 0.831 & 0.816 & 0.819 & 0.818 \\
\hline$F_{n}(0.6)$ & 0.6 & 0.748 & 0.729 & 0.732 & 0.732 \\
\hline $\mathrm{F}_{\mathrm{n}}(0.5)$ & 0.5 & 0.657 & 0.634 & 0.638 & 0.638 \\
\hline$F_{n}(0.4)$ & 0.4 & 0.556 & 0.530 & 0.534 & 0.534 \\
\hline$F_{n}(0.3)$ & 0.3 & 0.440 & 0.414 & 0.418 & 0.418 \\
\hline$F_{n}(0.2)$ & 0.2 & 0.310 & 0.288 & 0.291 & 0.291 \\
\hline$F_{n}(0.1)$ & 0.1 & 0.166 & 0.151 & 0.154 & 0.153 \\
\hline$\phi_{n}(0.9) \dagger$ & 2.775 & 2.857 & 2.841 & 2.844 & 2.850 \\
\hline${ }^{2} \phi_{n}(0.8)$ & 3.535 & 3.592 & 3.575 & 3.578 & 3.573 \\
\hline${ }^{2} \phi_{n}(0.7)$ & 4.017 & 4.034 & 4.021 & 4.023 & 4.016 \\
\hline${ }^{2} \phi_{n}(0.6)$ & 4.444 & 4.407 & 4.400 & 4.401 & 4.393 \\
\hline${ }^{2} \phi_{n}(0.5)$ & 4.919 & 4.800 & 4.803 & 4.803 & 4.794 \\
\hline${ }^{2} \phi_{n}(0.4)$ & 5.577 & 5.317 & 5.338 & 5.335 & 5.328 \\
\hline${ }^{2} \phi_{n}(0.3)$ & 6.361 & 5.900 & 5.949 & 5.941 & 5.939 \\
\hline${ }^{2} \phi_{n}(0.2)$ & 7.008 & 6.357 & 6.433 & 6.421 & 6.415 \\
\hline$)^{2} \phi_{n}(0.1)$ & 7.817 & 6.892 & 7.011 & 6.992 & 6.987 \\
\hline $2^{2} \quad s_{n} \quad \dagger$ & 1.3236 & 1.2466 & 1.2547 & 1.2534 & 1.2538 \\
\hline
\end{tabular}


TABLE 2

Comparison of the first four approximations with exact solution

for sorption in the 'linear' soil, taking $F=\theta$.

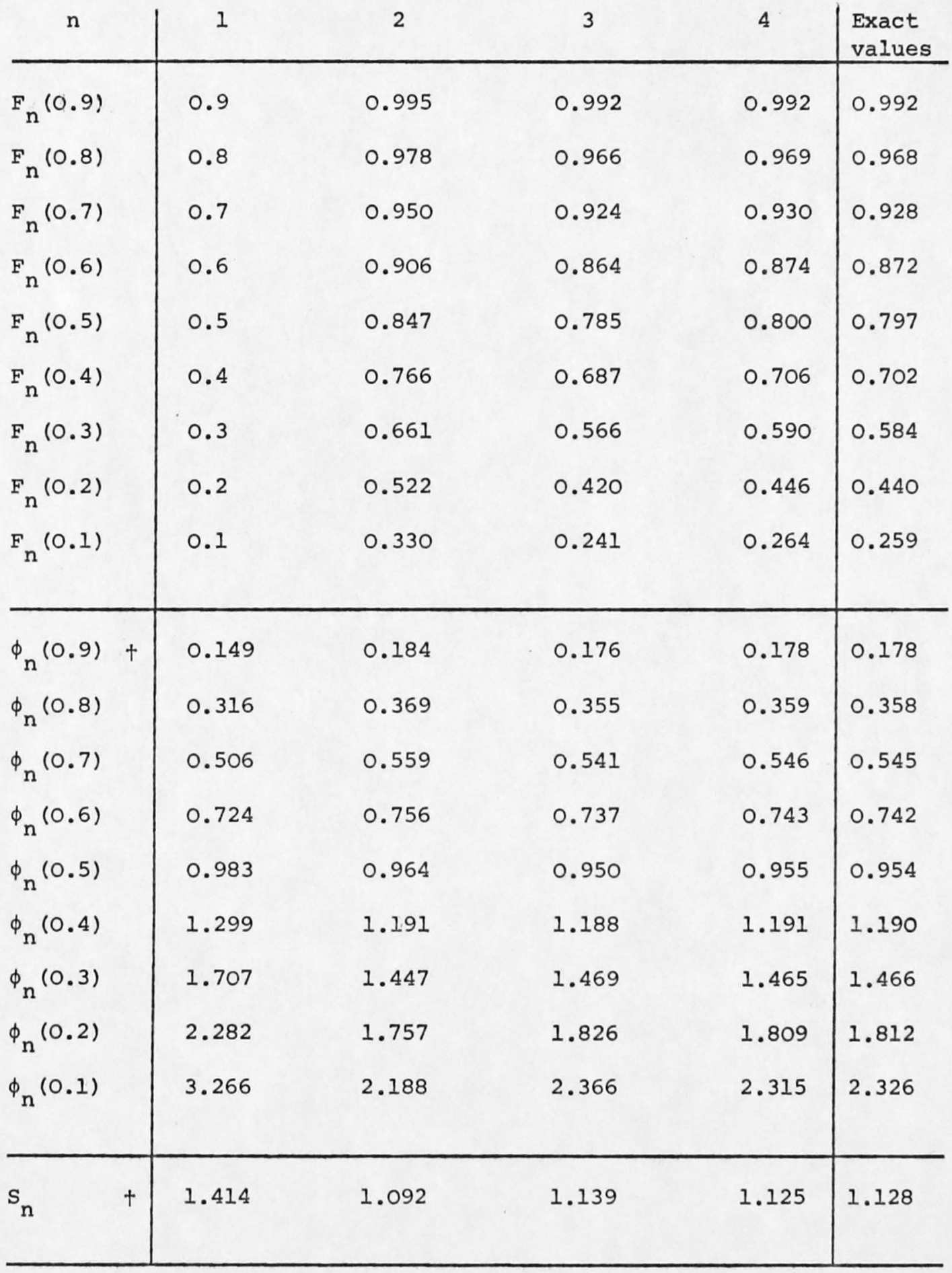

+ Unit of $\phi_{\mathrm{n}}$ and $\mathrm{s}_{\mathrm{n}}$ is $\mathrm{cm} \mathrm{sec}^{-\frac{1}{2}}$ 
Tables:

TABLE 1

Rate of convergence of iterative scheme (16), as measured by

$n_{\star}$, for family of D-functions defined by equation (19).

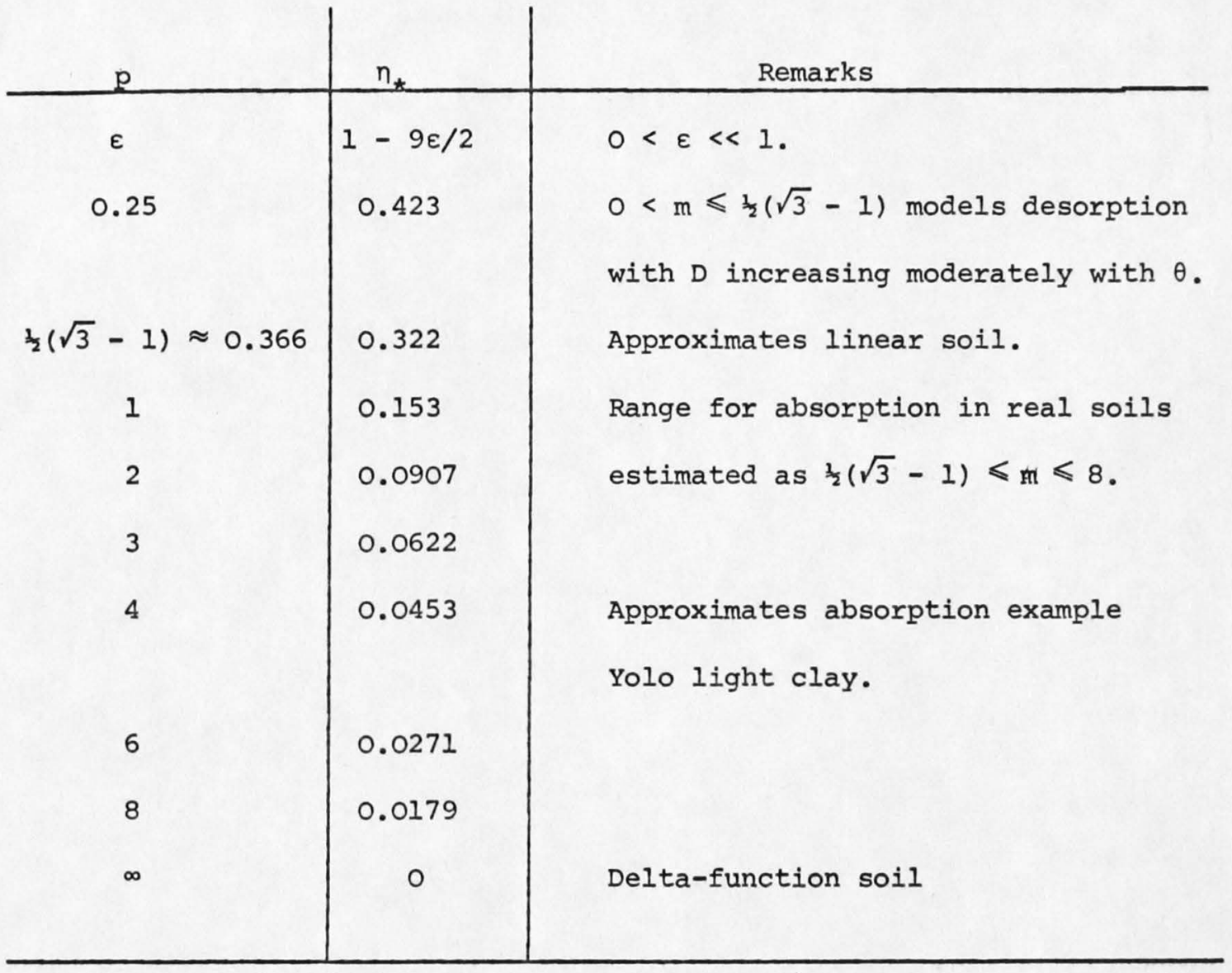




\section{Figure Legends}

FIG. 1. The first four approximations $\phi_{1}, \phi_{2}, \phi_{3}, \phi_{4}$ for sorption in the linear soil, compared with the exact solution $\phi$. $\theta$ is volumetric moisture content; $\phi(\theta)$ and $\phi_{n}(\theta)$ are moisture profiles in reduced form. Note that $\phi_{4}$ is indistinguishable from $\phi$.

FIG. 2. The first three approximations $\phi_{1}, \phi_{2}, \phi_{3}$ for absorption in Yolo light clay, compared with the exact solution $\phi . \theta$ is volumetric moisture content; $\phi(\theta)$ and $\phi_{n}(\theta)$ are moisture profiles in reduced form. Note that $\phi_{3}$ is indistinguishable from $\phi$.

FIG. 3. Infiltration in Yolo light clay. Comparisons of estimates of moisture profile at time $t=10^{4} \mathrm{sec} . \quad z$ is depth from surface, $\theta$ the volumetric moisture content. The curve denotes the four-term series solution of Philip (1957b). 1A: first approximation based on $\mathrm{F}_{1 \mathrm{~A}}$. 1B: first approximation based on $\mathrm{F}_{1 \mathrm{~B}}$. 1C: first approximation based on F $1 \mathrm{C}$. IP: Parlange's first approximation. 2P: Parlange's second approximation.

FIG. 4. As Figure 3 for $t=10^{5} \mathrm{sec}$.

FIG. 5. As Figure 3 for $t=10^{6} \mathrm{sec}$. 